\title{
PIFA-Mediated Tandem Hofmann-Type Rearrangement and Cyclization Reaction of $\alpha$-Acyl- $\beta$-aminoacrylamides: Access to Polysubstituted Oxazol-2(3H)-ones
}

Baibin Li, ${ }^{\S a, b}$ Jingwen Yuan, ${ }^{\S}$ X Xuebei Ye, ${ }^{\text {a,b }}$ Rui Zhang, , , a Jiacheng Li, a,b Yu Wang, Jiana Hu, a,b and Dewen Dong*, a,b

${ }^{\text {a }}$ CAS Key Laboratory of High-Performance Synthetic Rubber and Its Composite Materials, Changchun Institute of Applied Chemistry, Chinese Academy of Sciences, Changchun 130022, P. R. China

${ }^{\mathrm{b}}$ University of Science and Technology of China, Hefei 230026, P. R. China

$\S$ These authors contributed equally

E-mail: ariel@ciac.ac.cn; $\underline{\text { dwdong@ciac.ac.cn }}$

Supporting Information

\section{Contents}

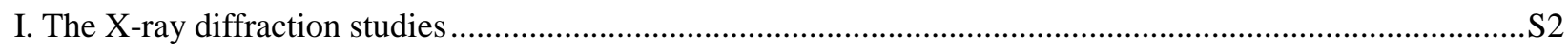

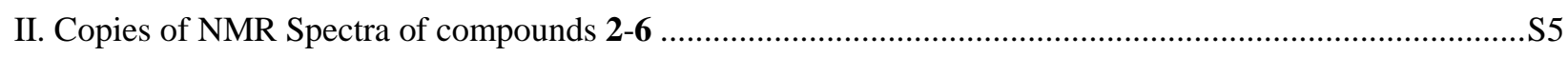




\section{The X-ray diffraction studies}

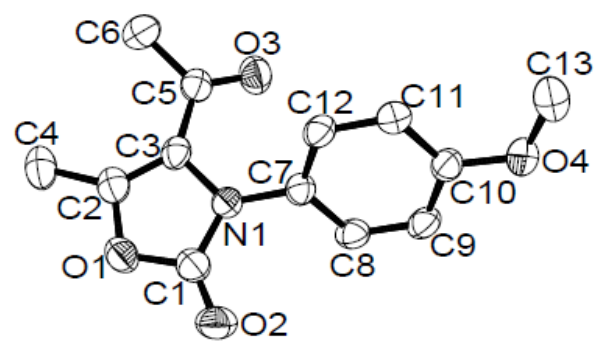

Figure S1 X-ray crystal structure of 4a. Hydrogen atoms have been omitted for the sake of simplicity. The ORTEP plot is at the $50 \%$ probability level.

Note: The crystals of $\mathbf{4 a}$ were obtained by slow evaporation of a solution of the isolated compound in a mixed solvents of petroleum ether and ethyl acetate $(5: 1, \mathrm{v} / \mathrm{v})$ at room temperature. The single crystal X-ray diffraction experiments were carried out using a Bruker Smart APEX diffractometer with CCD detector and graphite monochromator, Mo K $\alpha$ radiation $(\lambda=0.71073 \AA)$. The intensity data were recorded with $\omega$ scan mode $(273.15 \mathrm{~K})$. Lorentz, polarization factors were made for the intensity data and absorption corrections were performed using SADABS program. ${ }^{[\mathrm{S} 1]}$ The crystal structure was determined using the SHELXTL program and refined using full matrix least squares. ${ }^{[\mathrm{S} 2]}$ All non-hydrogen atoms were assigned with anisotropic displacement parameters, whereas hydrogen atoms were placed at calculated positions theoretically and included in the final cycles of refinement in a riding model along with the attached carbons. The detailed data can be obtained free of charge from the Cambridge Crystallographic Data Center via www.ccdc.cam.ac.uk/ data_request/cif with CCDC 2078087 for the compound. Crystal data and details on data collection and refinement are summarized in Table S1-S3. 
Table S1 Crystal data and structure refinement for $\mathbf{4 a}$

\begin{tabular}{|c|c|}
\hline Identification code & CCDC 2078087 \\
\hline Empirical formula & $\mathrm{C}_{13} \mathrm{H}_{13} \mathrm{NO}_{4}$ \\
\hline Formula weight & 247.24 \\
\hline Temperature/K & 273.15 \\
\hline Crystal system & monoclinic \\
\hline Space group & $\mathrm{P} 2{ }_{1} / \mathrm{n}$ \\
\hline $\mathrm{a} / \AA$ & $7.7468(7)$ \\
\hline $\mathrm{b} / \AA$ & $12.7900(12)$ \\
\hline $\mathrm{c} / \AA$ & $11.8138(11)$ \\
\hline$\alpha /^{\circ}$ & 90.00 \\
\hline$\beta /{ }^{\circ}$ & $90.856(2)$ \\
\hline$\gamma /{ }^{\circ}$ & 90.00 \\
\hline Volume $/ \AA^{3}$ & $1170.40(19)$ \\
\hline $\mathrm{Z}$ & 4 \\
\hline$\rho_{\text {calc }} \mathrm{g} / \mathrm{cm}^{3}$ & 1.403 \\
\hline$\mu / \mathrm{mm}^{-1}$ & 0.105 \\
\hline $\mathrm{F}(000)$ & 520.0 \\
\hline Crystal size $/ \mathrm{mm}^{3}$ & $0.26 \times 0.22 \times 0.2$ \\
\hline Radiation & $\operatorname{MoK} \alpha(\lambda=0.71073)$ \\
\hline $2 \Theta$ range for data collection $/{ }^{\circ}$ & 4.7 to 52.47 \\
\hline Index ranges & $-8 \leq \mathrm{h} \leq 9,-15 \leq \mathrm{k} \leq 15,-11 \leq \mathrm{l} \leq 14$ \\
\hline Reflections collected & 6548 \\
\hline Independent reflections & $2388\left[\mathrm{R}_{\mathrm{int}}=0.0190, \mathrm{R}_{\mathrm{sigma}}=0.0234\right]$ \\
\hline Data/restraints/parameters & $2388 / 0 / 166$ \\
\hline Goodness-of-fit on $\mathrm{F}^{2}$ & 1.051 \\
\hline Final $\mathrm{R}$ indexes $[\mathrm{I}>=2 \sigma(\mathrm{I})]$ & $\mathrm{R}_{1}=0.0416, \mathrm{wR}_{2}=0.1119$ \\
\hline Final $\mathrm{R}$ indexes [all data] & $\mathrm{R}_{1}=0.0495, \mathrm{wR}_{2}=0.1179$ \\
\hline Largest diff. peak/hole / e $\AA^{-3}$ & $0.39 /-0.18$ \\
\hline
\end{tabular}


Table S2 Bond lengths

\begin{tabular}{|c|c|c|c|c|c|}
\hline Atom & Atom & Length/Å & Atom & Atom & Length/Å \\
\hline $\mathrm{O} 1$ & $\mathrm{C} 1$ & $1.3785(18)$ & C7 & $\mathrm{C} 8$ & $1.393(2)$ \\
\hline O1 & $\mathrm{C} 2$ & $1.3860(19)$ & $\mathrm{C} 3$ & $\mathrm{C} 5$ & $1.474(2)$ \\
\hline $\mathrm{O} 4$ & $\mathrm{C} 10$ & $1.3667(18)$ & $\mathrm{C} 3$ & $\mathrm{C} 2$ & $1.342(2)$ \\
\hline $\mathrm{O} 4$ & $\mathrm{C} 13$ & $1.426(2)$ & $\mathrm{C} 12$ & $\mathrm{C} 11$ & $1.386(2)$ \\
\hline $\mathrm{O} 3$ & $\mathrm{C} 5$ & $1.2123(19)$ & $\mathrm{C} 8$ & C9 & $1.376(2)$ \\
\hline $\mathrm{O} 2$ & $\mathrm{C} 1$ & $1.2059(19)$ & $\mathrm{C} 10$ & $\mathrm{C} 11$ & $1.391(2)$ \\
\hline N1 & $\mathrm{C} 7$ & $1.4341(18)$ & $\mathrm{C} 10$ & C9 & $1.392(2)$ \\
\hline N1 & $\mathrm{C} 3$ & $1.4187(18)$ & $\mathrm{C} 5$ & C6 & $1.497(2)$ \\
\hline N1 & $\mathrm{C} 1$ & $1.3621(19)$ & $\mathrm{C} 2$ & $\mathrm{C} 4$ & $1.481(2)$ \\
\hline $\mathrm{C} 7$ & $\mathrm{C} 12$ & $1.379(2)$ & & & \\
\hline
\end{tabular}

Table S3 Bond angles

\begin{tabular}{cccccccc}
\hline Atom & Atom & Atom & Angle $^{\circ}$ & Atom & Atom & Atom & Angle $^{\circ}$ \\
\hline C1 & O1 & C2 & $108.79(11)$ & O4 & C10 & C9 & $115.90(13)$ \\
C10 & O4 & C13 & $117.01(12)$ & C11 & C10 & C9 & $119.70(14)$ \\
C3 & N1 & C7 & $127.50(12)$ & C12 & C11 & C10 & $119.73(14)$ \\
C1 & N1 & C7 & $121.83(12)$ & O3 & C5 & C3 & $120.39(14)$ \\
C1 & N1 & C3 & $108.95(12)$ & O3 & C5 & C6 & $121.51(15)$ \\
C12 & C7 & N1 & $120.46(13)$ & C3 & C5 & C6 & $118.09(14)$ \\
C12 & C7 & C8 & $119.94(14)$ & C8 & C9 & C10 & $120.30(13)$ \\
C8 & C7 & N1 & $119.58(12)$ & O2 & C1 & O1 & $122.71(14)$ \\
N1 & C3 & C5 & $122.22(12)$ & O2 & C1 & N1 & $130.67(15)$ \\
C2 & C3 & N1 & $106.73(13)$ & N1 & C1 & O1 & $106.62(13)$ \\
C2 & C3 & C5 & $130.49(12)$ & O1 & C2 & C4 & $114.40(13)$ \\
C7 & C12 & C11 & $120.39(13)$ & C3 & C2 & O1 & $108.83(13)$ \\
C9 & C8 & C7 & $119.92(14)$ & C3 & C2 & C4 & $136.75(16)$ \\
O4 & C10 & C11 & $124.39(14)$ & & & & \\
\hline
\end{tabular}

\section{References}

[S1] J. Huang, Y. Liang, W. Pan, Y. Yang, D. Dong, Org. Lett. 2007, 9, 5345.

[S2] H. A. Stefani, I. M. Costa, D. O. Silva, Synthesis 2000, 1526.

[S1] R. H. Blessing, Acta Crystallogr. Sect. A: Found. Crystallogr. 1995, 51, 33.

[S2] G. M. Sheldrick, SHELXTL, Version 5.1, Bruker Analytical X-ray System. Inc.: Madison, WI 1997. 


\section{NMR Spectra}

${ }^{1} \mathrm{H}$ NMR spectrum of $\mathbf{2 h}\left(300 \mathrm{MHz}, \mathrm{CDCl}_{3}\right)$

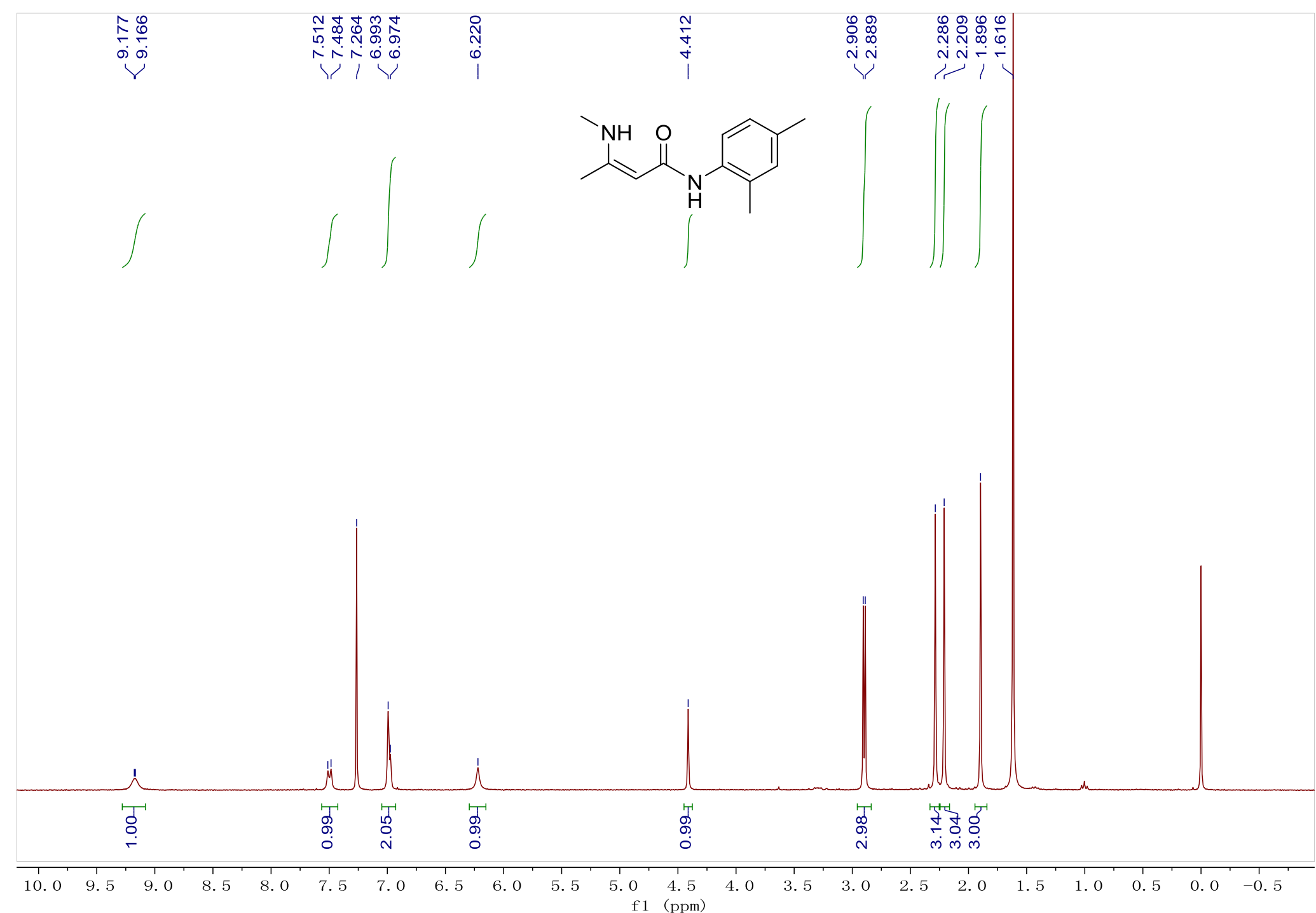


${ }^{13} \mathrm{C}\left\{{ }^{1} \mathrm{H}\right\}$ NMR spectrum of $\mathbf{2 h}\left(75 \mathrm{MHz}, \mathrm{CDCl}_{3}\right)$

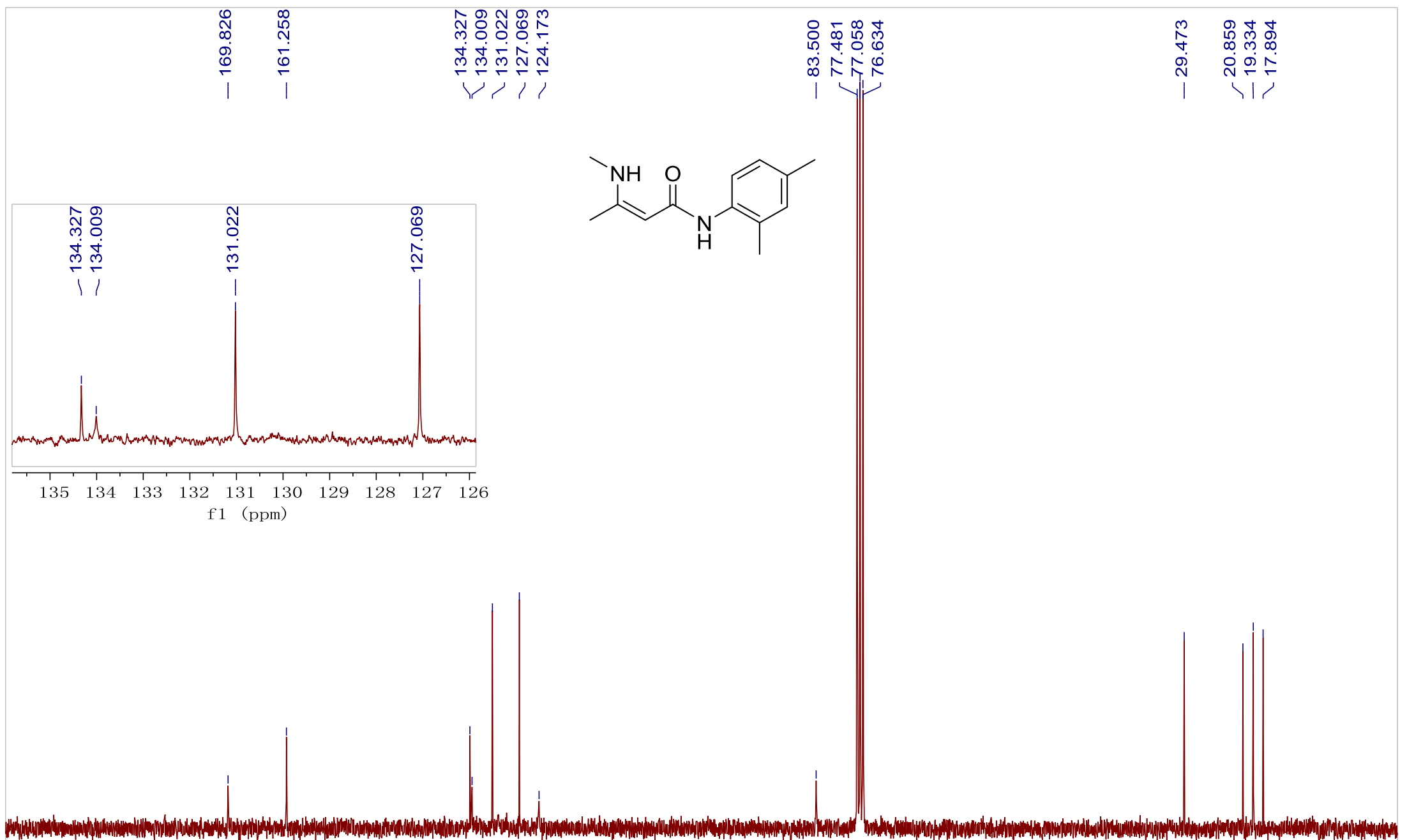

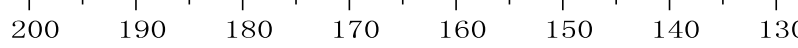

120

$110 \begin{gathered}100 \\ \mathrm{f} 1 \quad(\mathrm{ppm})\end{gathered}$ 
${ }^{1} \mathrm{H}$ NMR spectrum of $\mathbf{2 i}\left(300 \mathrm{MHz}, \mathrm{CDCl}_{3}\right.$ )

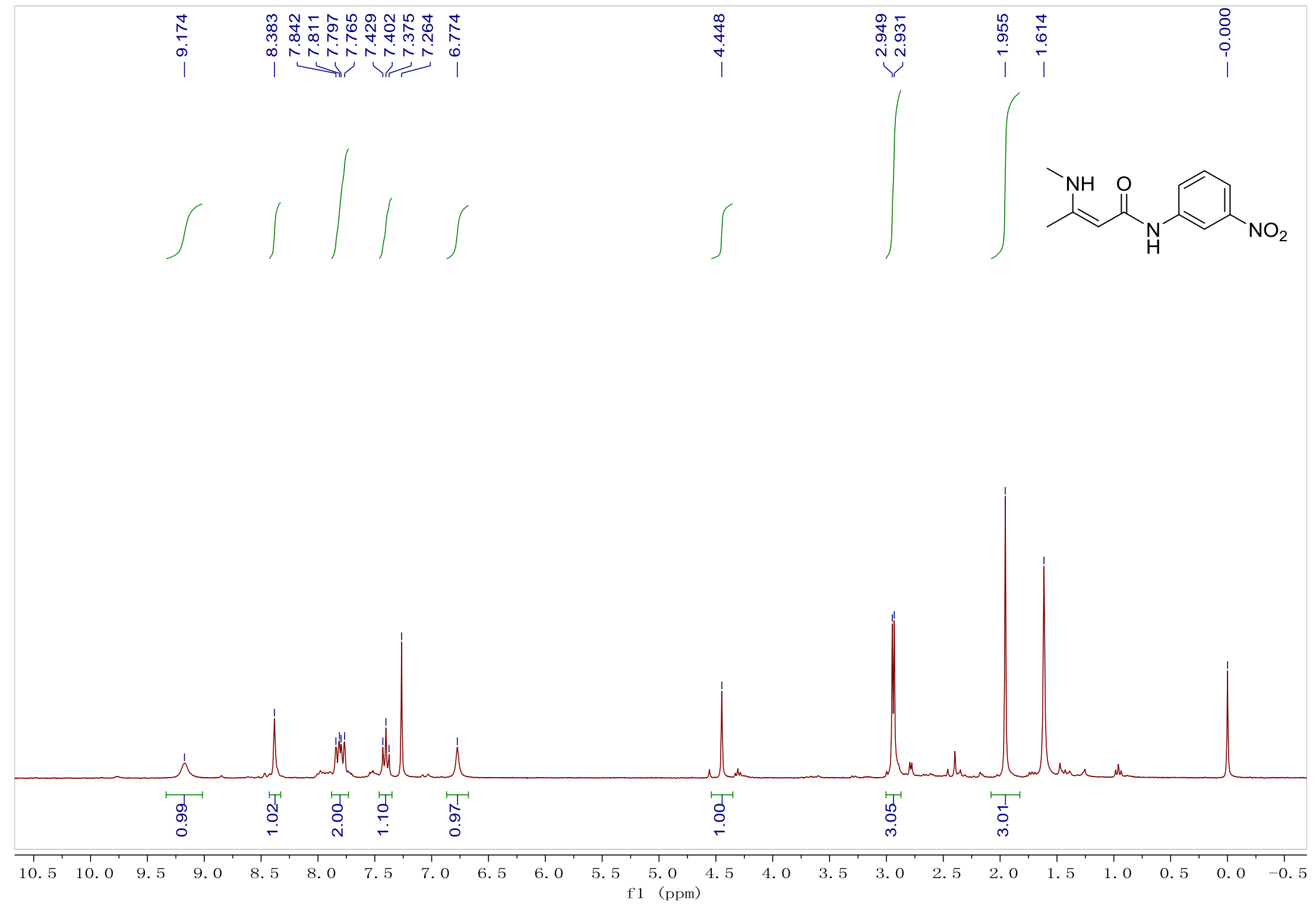


${ }^{13} \mathrm{C}\left\{{ }^{1} \mathrm{H}\right\}$ NMR spectrum of $2 \mathbf{i}\left(75 \mathrm{MHz}, \mathrm{CDCl}_{3}\right)$

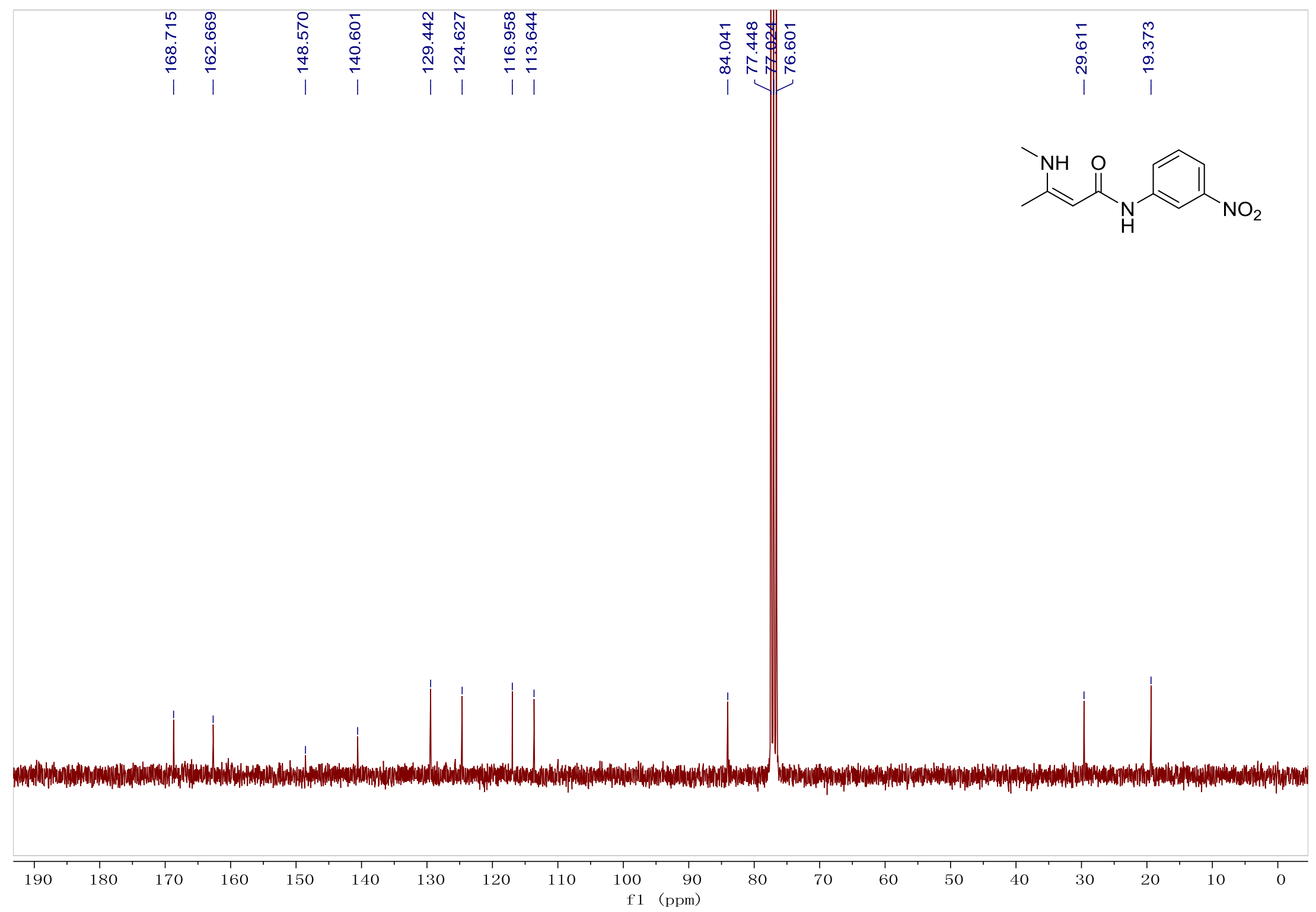




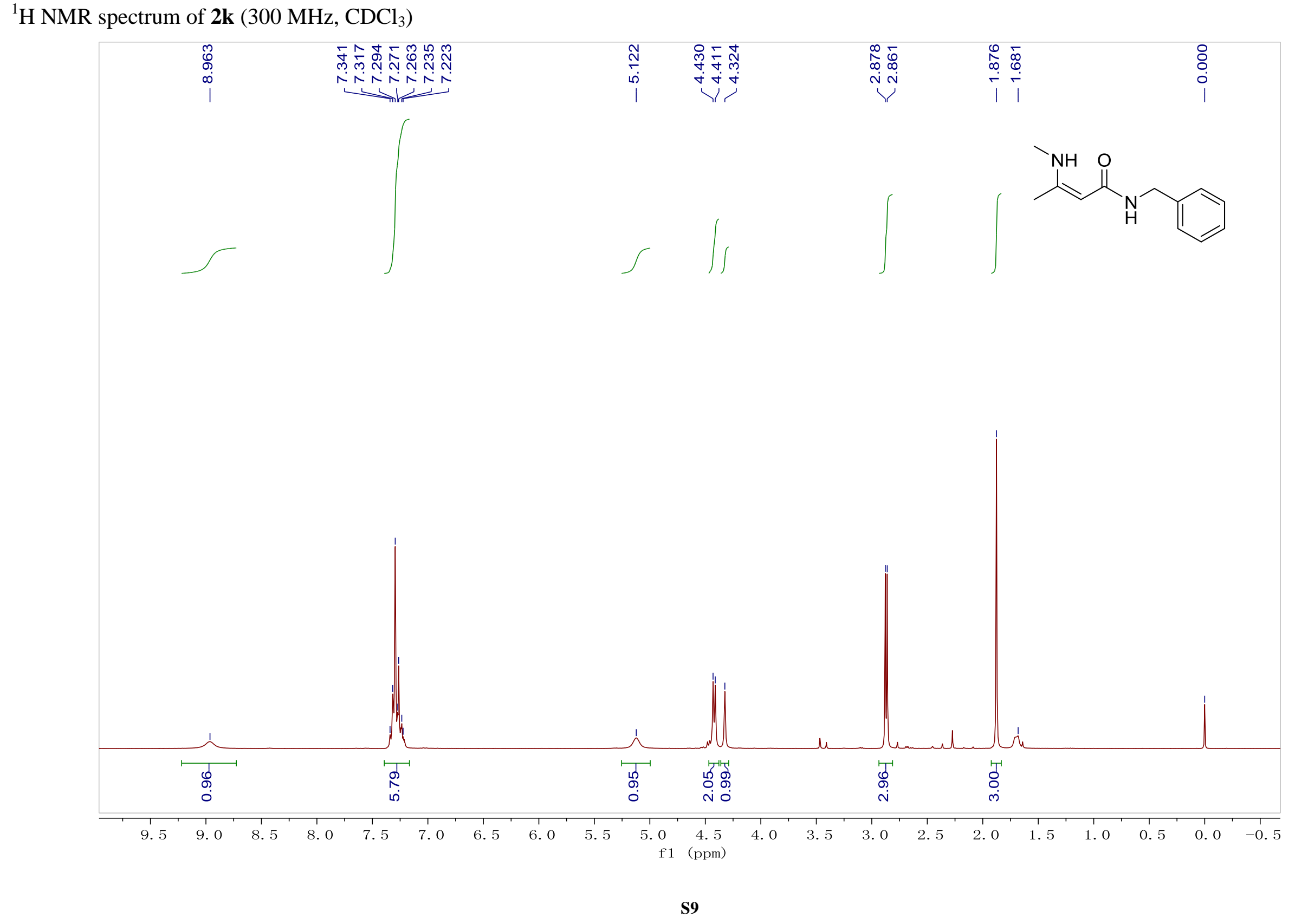


${ }^{13} \mathrm{C}\left\{{ }^{1} \mathrm{H}\right\}$ NMR spectrum of $\mathbf{2 k}\left(75 \mathrm{MHz}, \mathrm{CDCl}_{3}\right)$
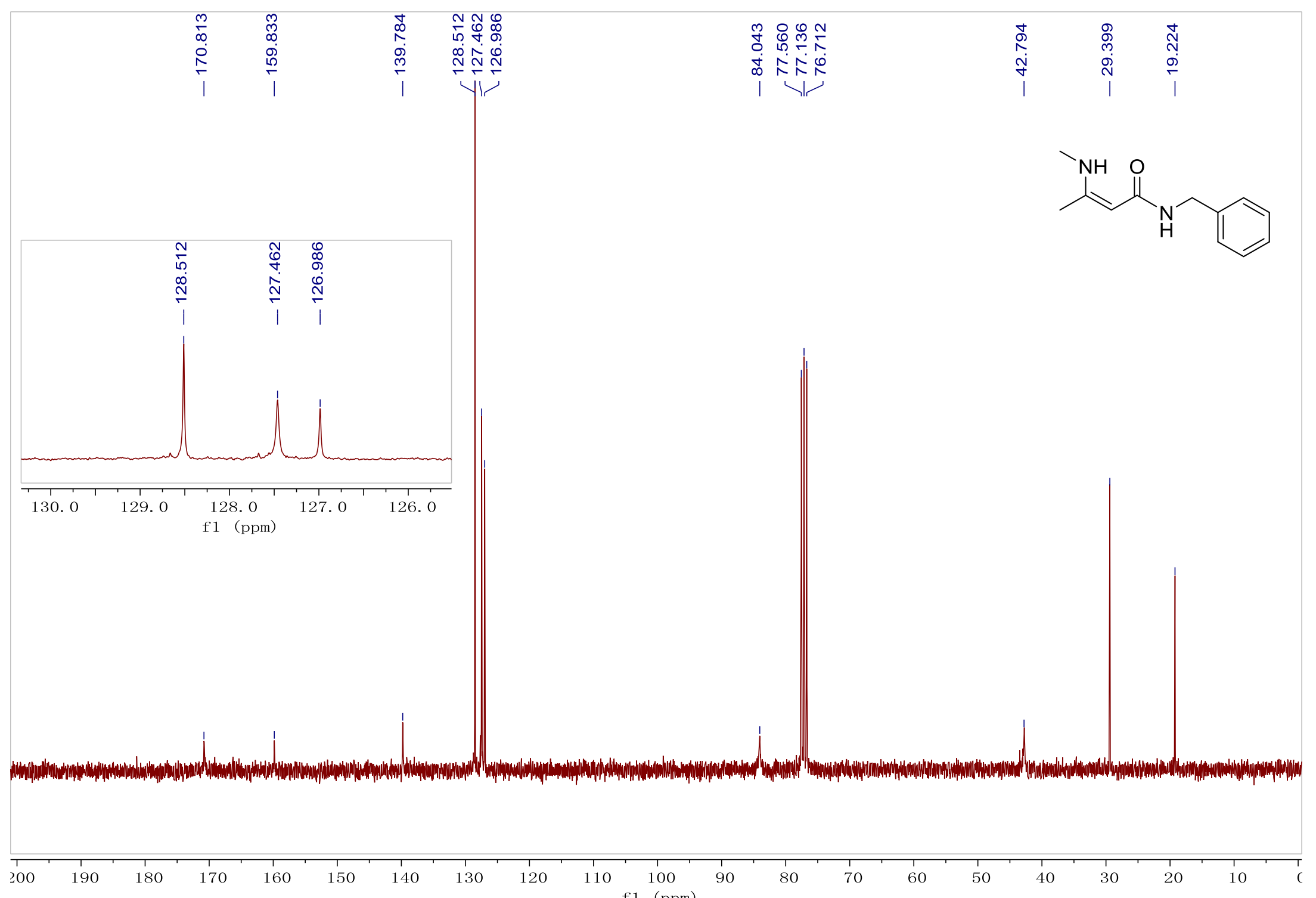
${ }^{1} \mathrm{H}$ NMR spectrum of $\mathbf{2 m}\left(300 \mathrm{MHz}, \mathrm{CDCl}_{3}\right)$

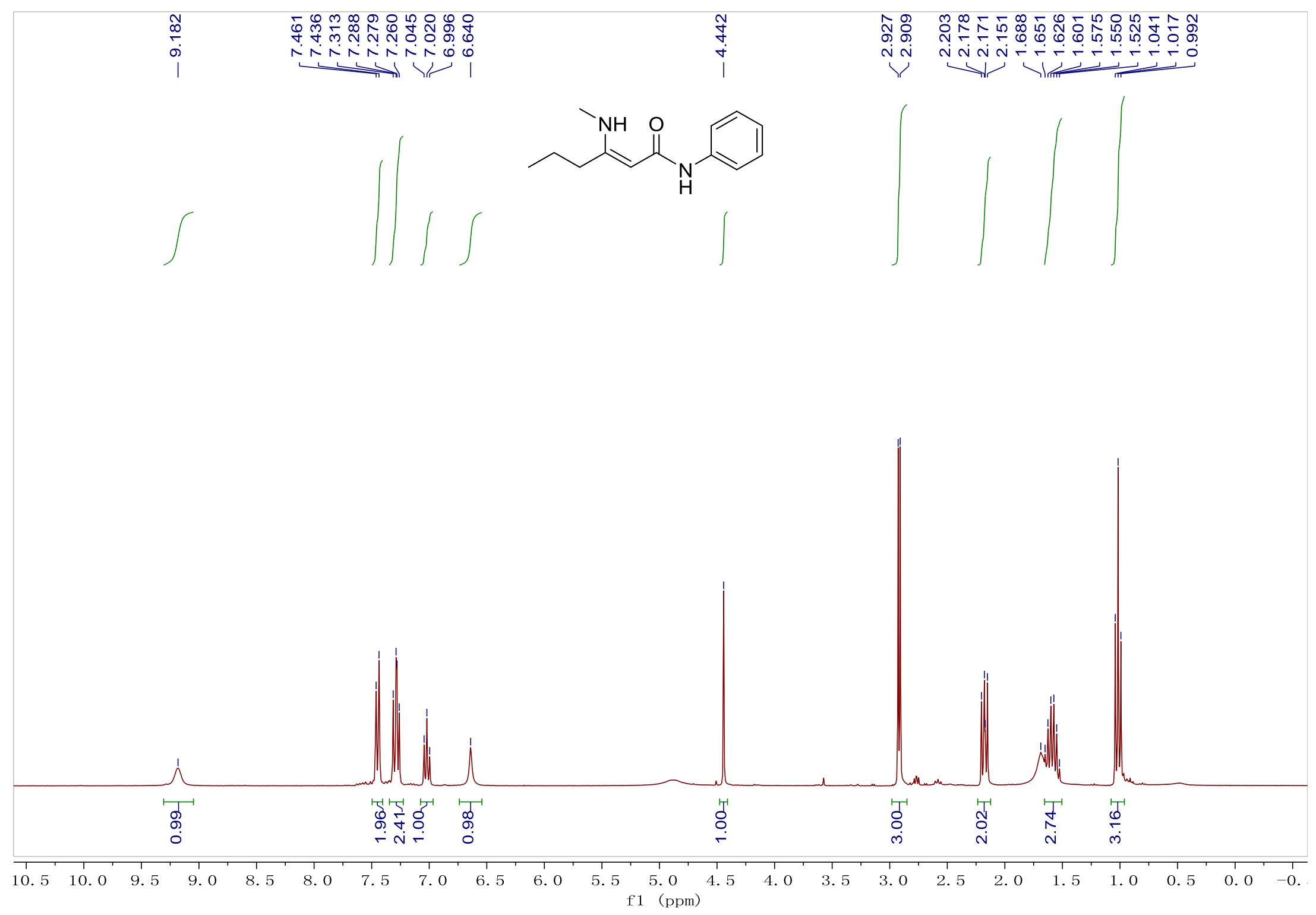

S11 
${ }^{13} \mathrm{C}\left\{{ }^{1} \mathrm{H}\right\}$ NMR spectrum of $\mathbf{2 m}\left(75 \mathrm{MHz}, \mathrm{CDCl}_{3}\right)$

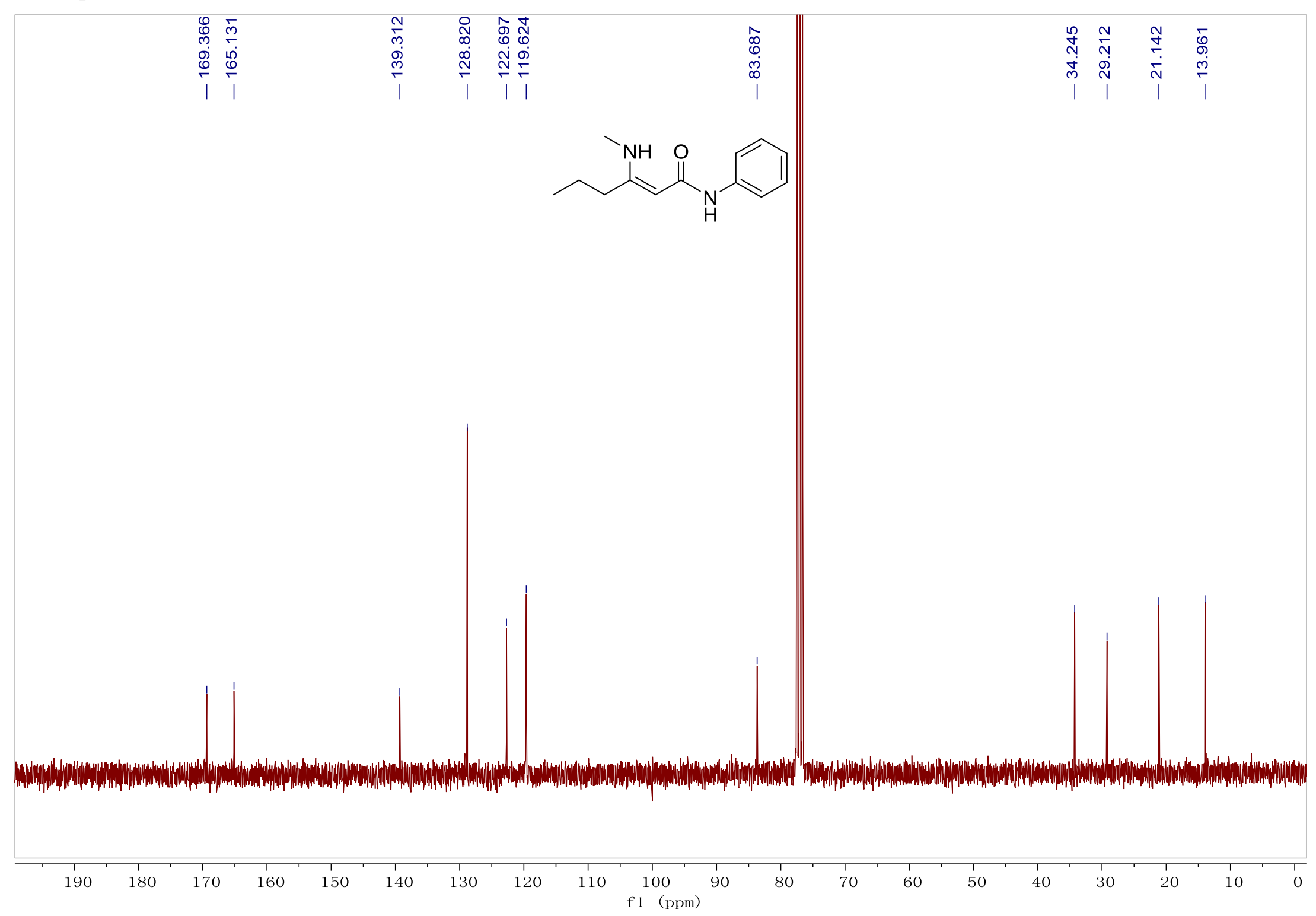


${ }^{1} \mathrm{H}$ NMR spectrum of $\mathbf{3 a}\left(300 \mathrm{MHz}, \mathrm{CDCl}_{3}\right)$

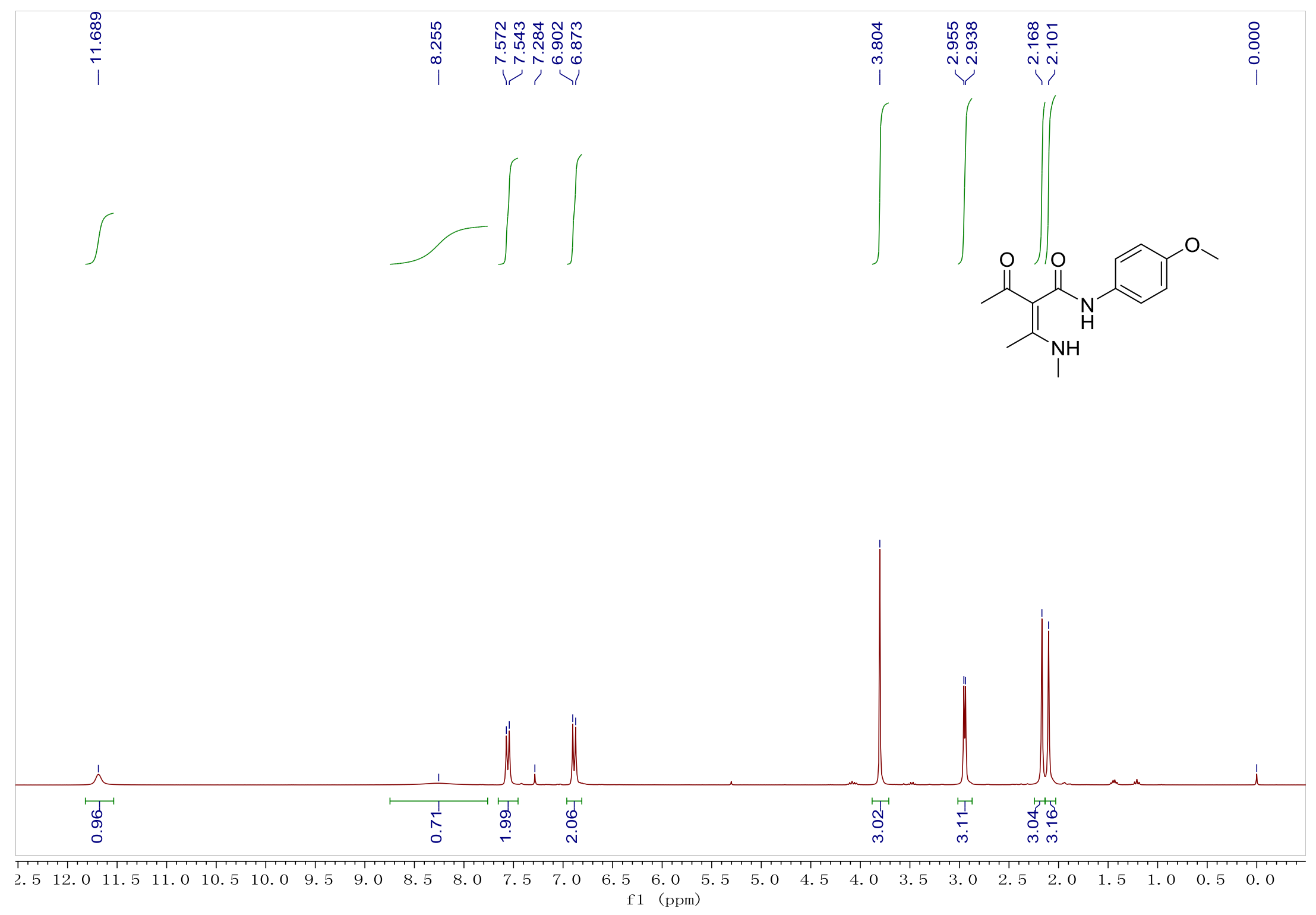


${ }^{13} \mathrm{C}\left\{{ }^{1} \mathrm{H}\right\}$ NMR spectrum of $\mathbf{3 a}\left(75 \mathrm{MHz}, \mathrm{CDCl}_{3}\right)$

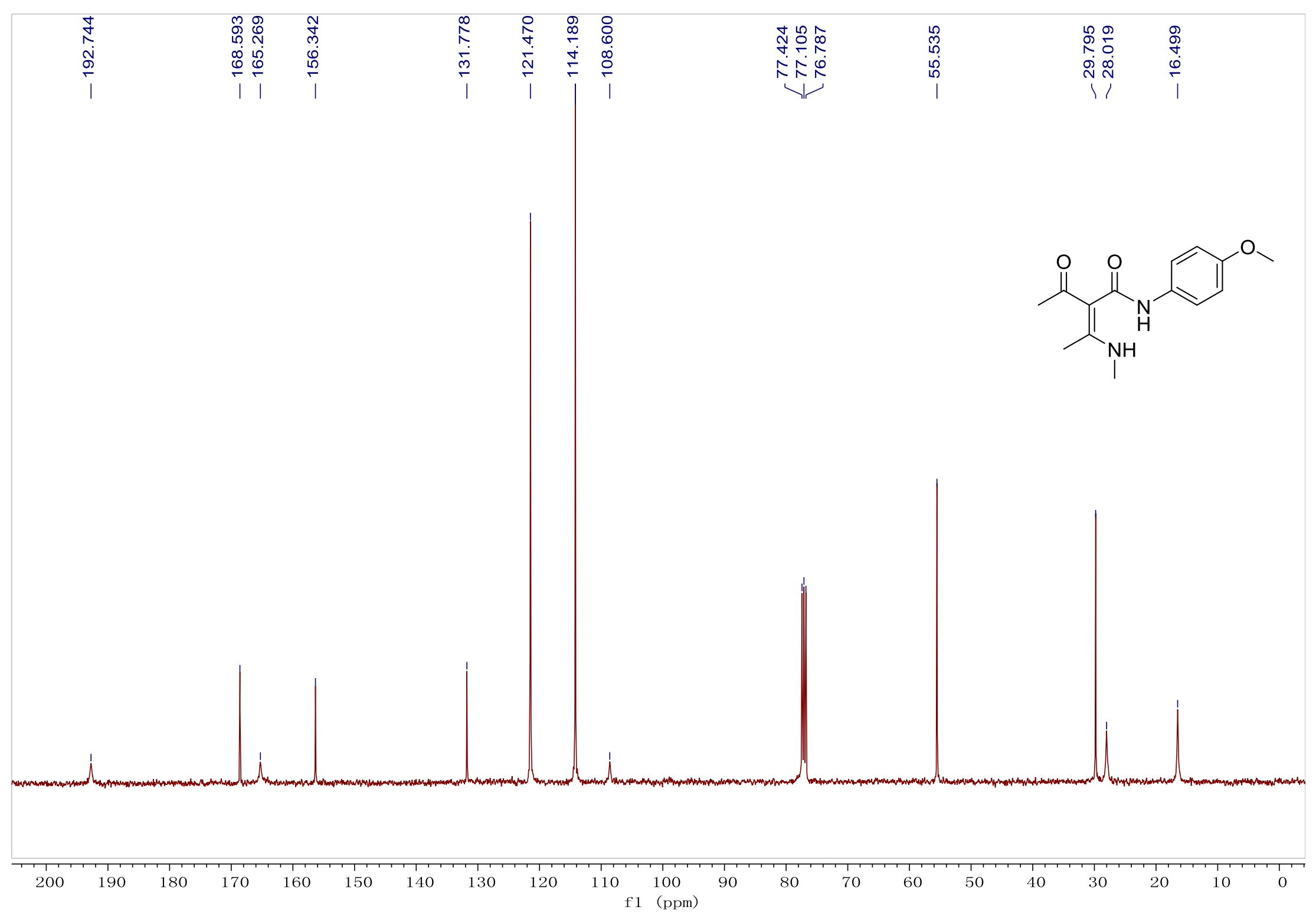


${ }^{1} \mathrm{H}$ NMR spectrum of $\mathbf{3 b}\left(300 \mathrm{MHz}, \mathrm{CDCl}_{3}\right)$

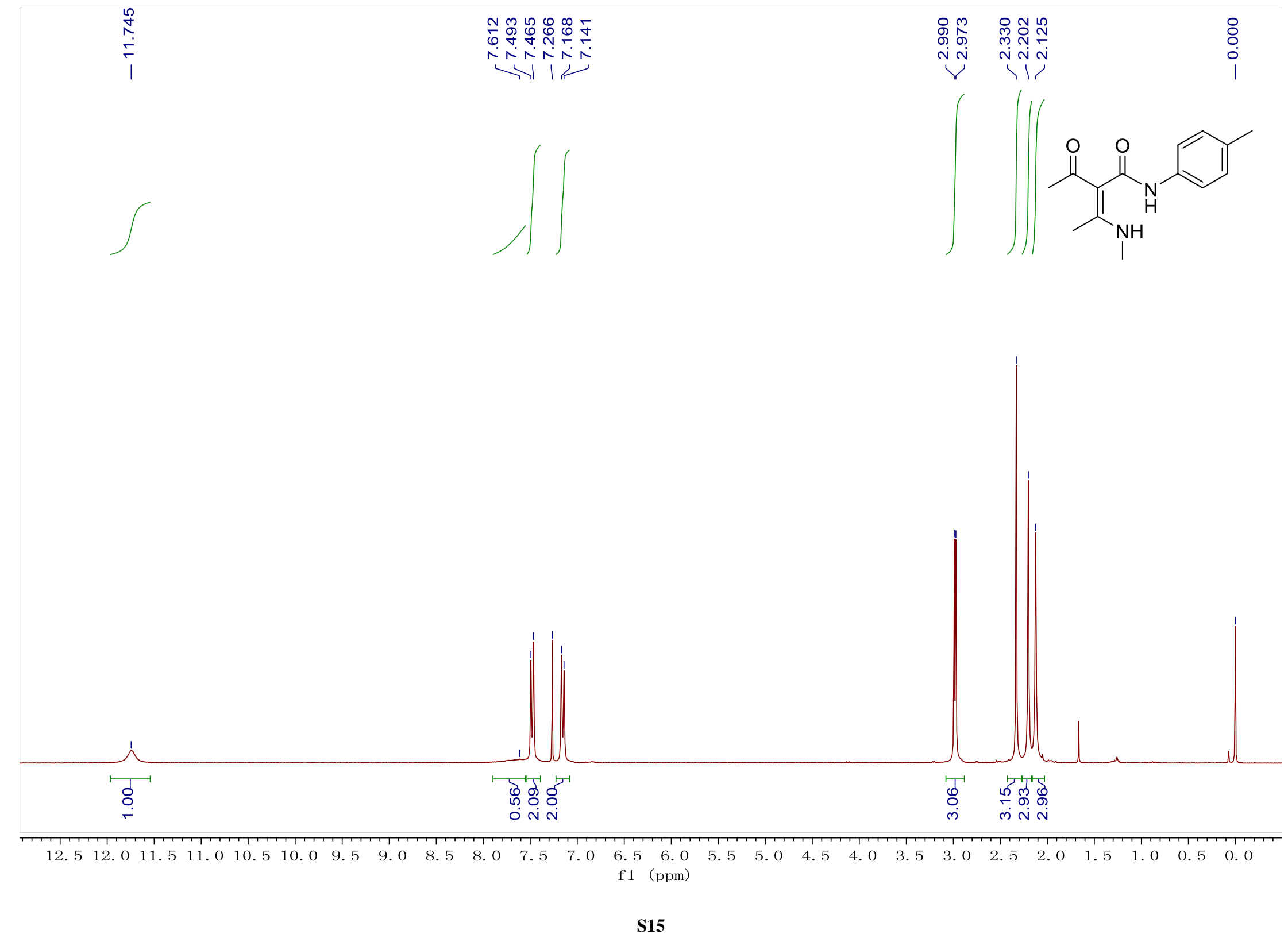


${ }^{13} \mathrm{C}\left\{{ }^{1} \mathrm{H}\right\}$ NMR spectrum of $\mathbf{3 b}\left(75 \mathrm{MHz}, \mathrm{CDCl}_{3}\right)$

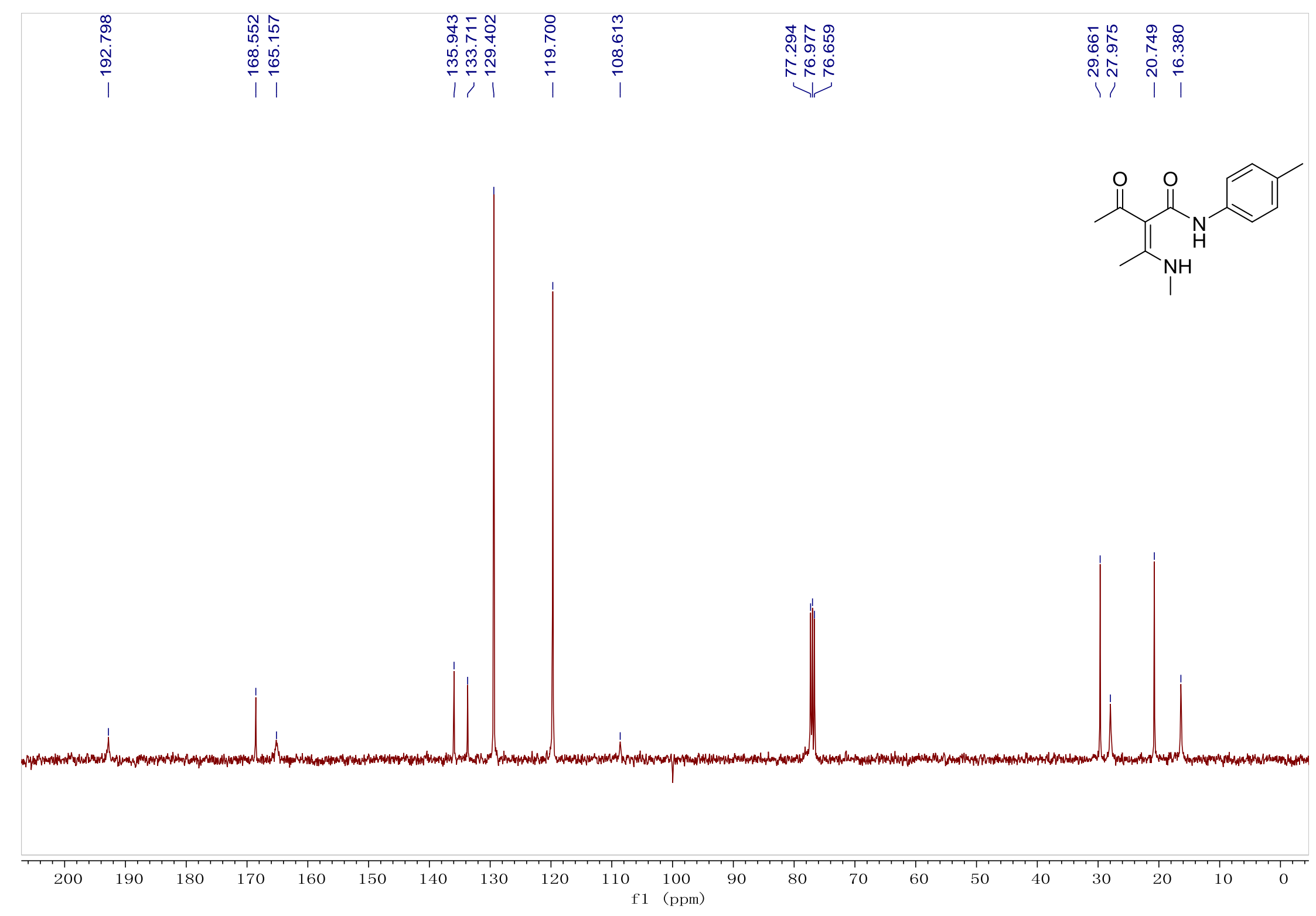


${ }^{1} \mathrm{H}$ NMR spectrum of $\mathbf{3 c}\left(300 \mathrm{MHz}, \mathrm{CDCl}_{3}\right)$

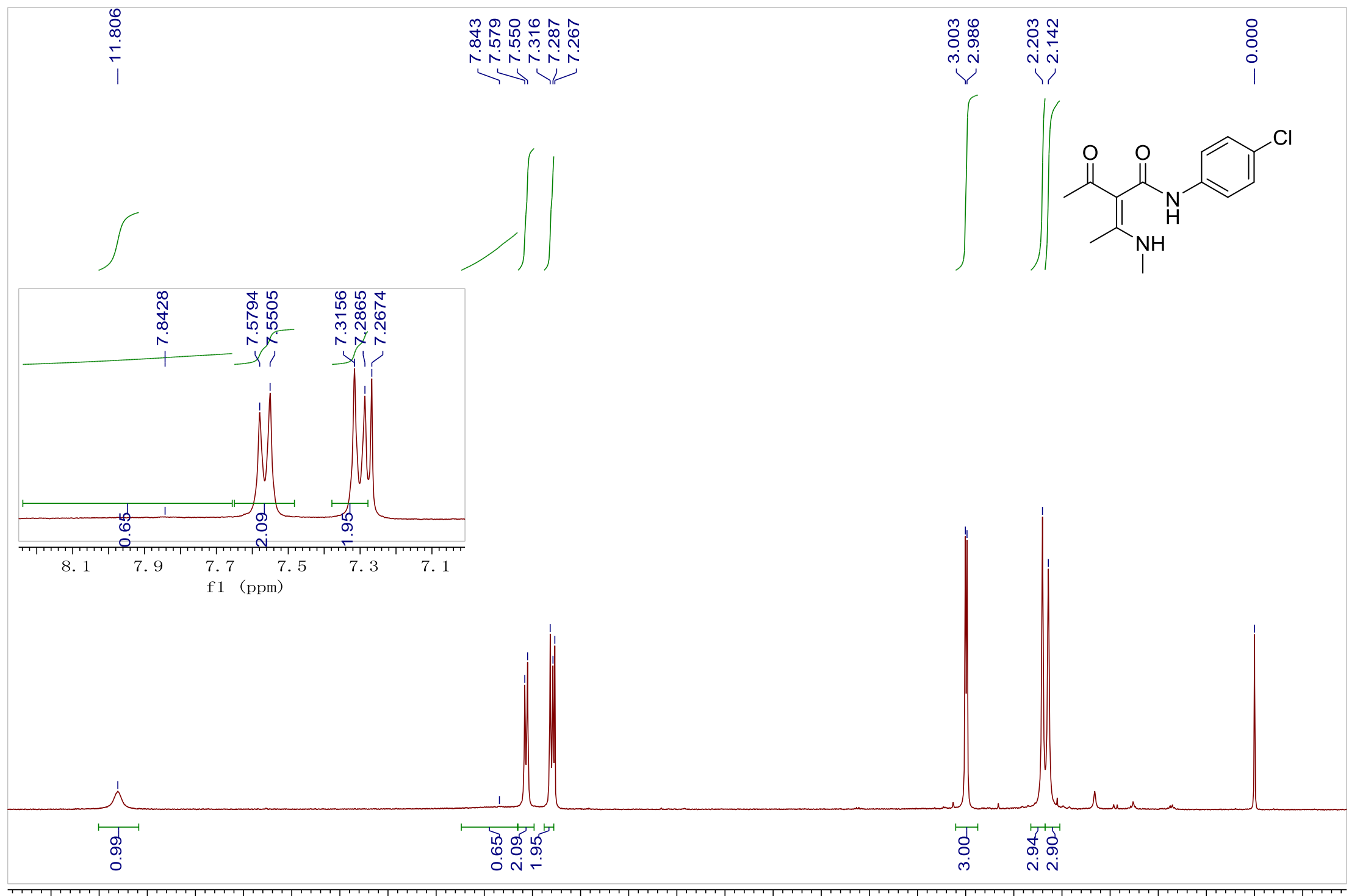

$\begin{array}{llllllllllllllllllllllllllll}12.5 & 12.0 & 11.5 & 11.0 & 10.5 & 10.0 & 9.5 & 9.0 & 8.5 & 8.0 & 7.5 & 7.0 & 6.5 & 6.0 & 5.5 & 5.0 & 4.5 & 4.0 & 3.5 & 3.0 & 2.5 & 2.0 & 1.5 & 1.0 & 0.5 & 0.0 & -0.5\end{array}$ f1 (ppm) 
${ }^{13} \mathrm{C}\left\{{ }^{1} \mathrm{H}\right\}$ NMR spectrum of $\mathbf{3 c}\left(100 \mathrm{MHz}, \mathrm{CDCl}_{3}\right)$

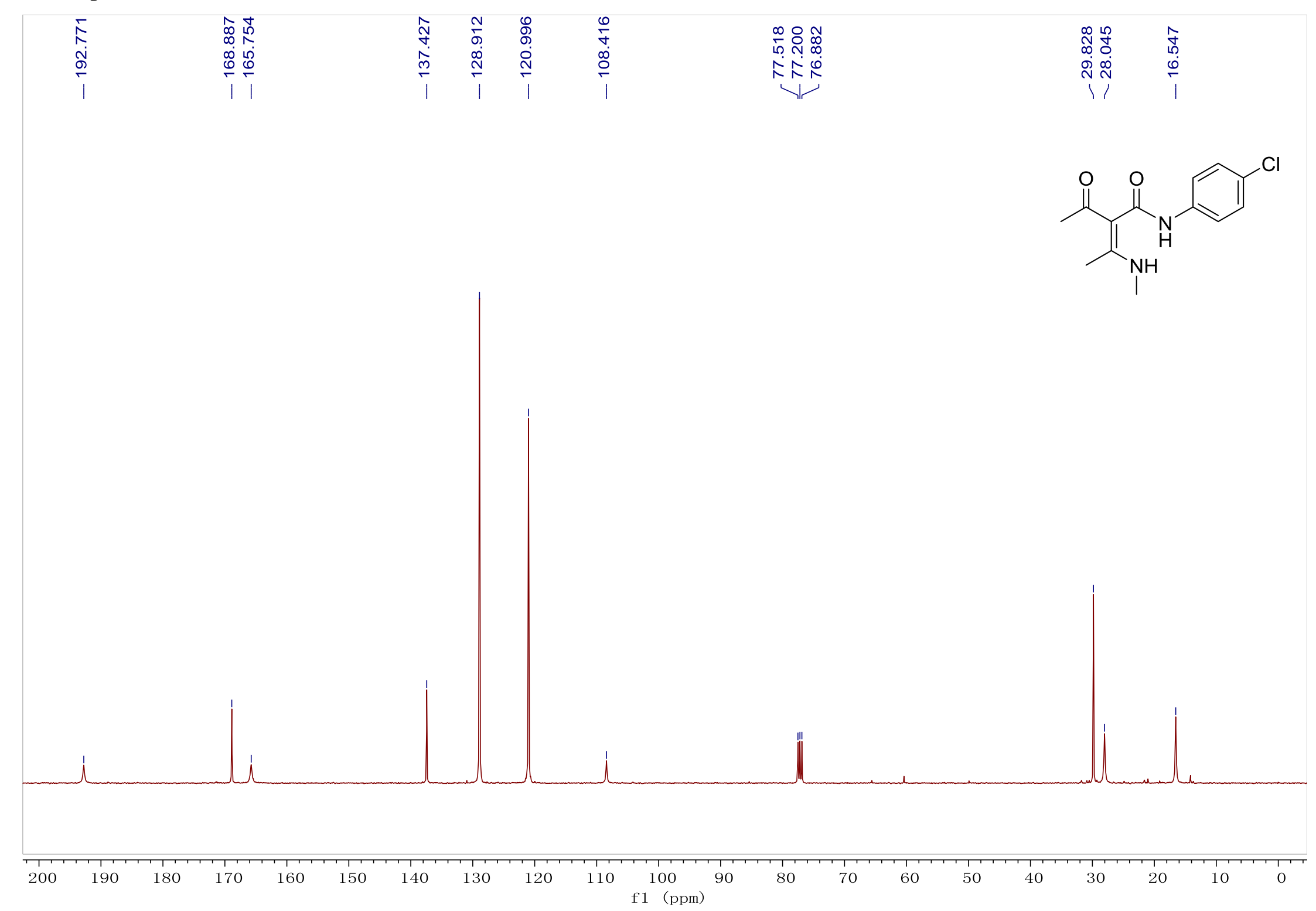


${ }^{1} \mathrm{H}$ NMR spectrum of $\mathbf{3 d}\left(300 \mathrm{MHz}, \mathrm{CDCl}_{3}\right)$

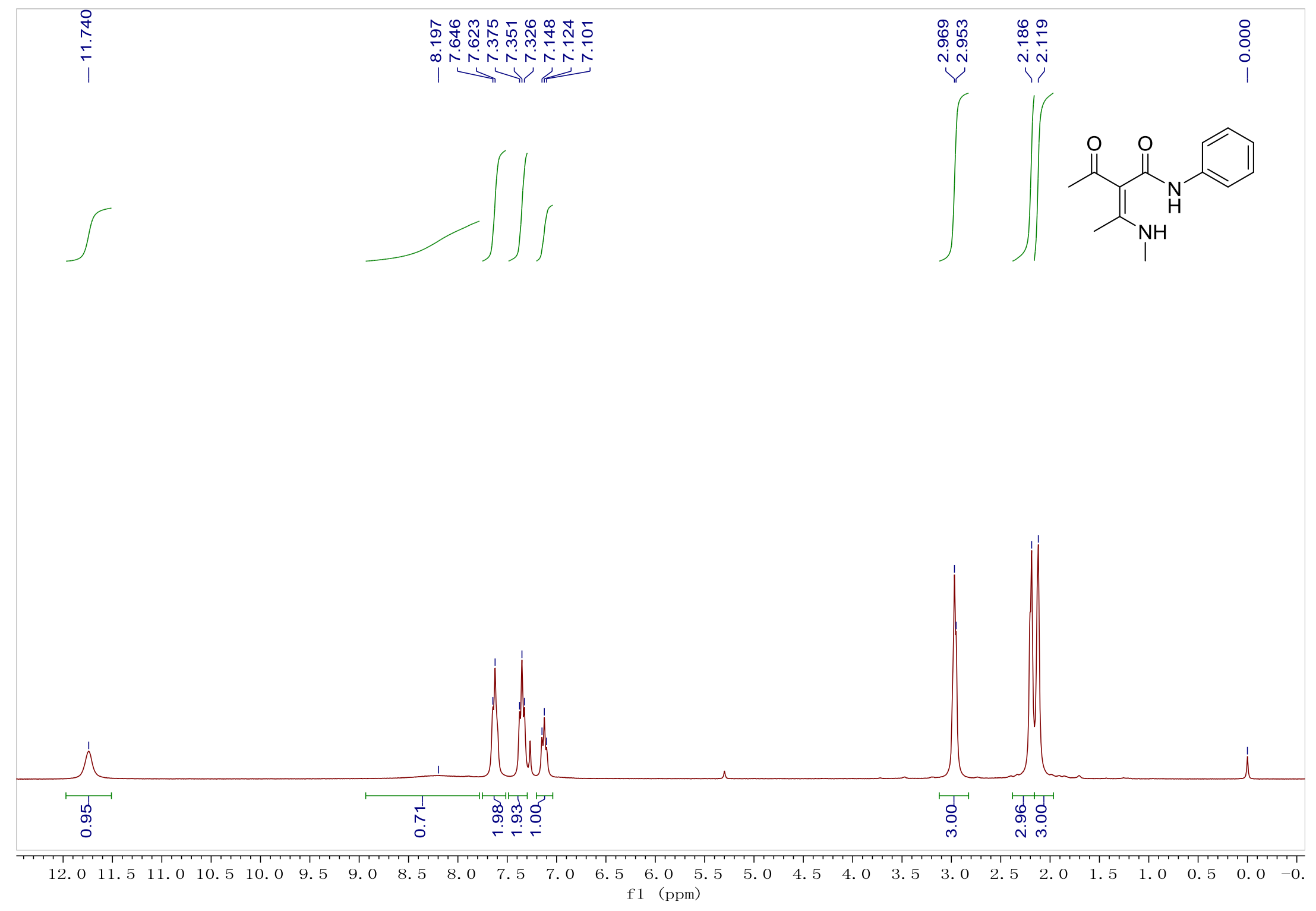


${ }^{13} \mathrm{C}\left\{{ }^{1} \mathrm{H}\right\}$ NMR spectrum of $\mathbf{3 d}\left(100 \mathrm{MHz}, \mathrm{CDCl}_{3}\right)$

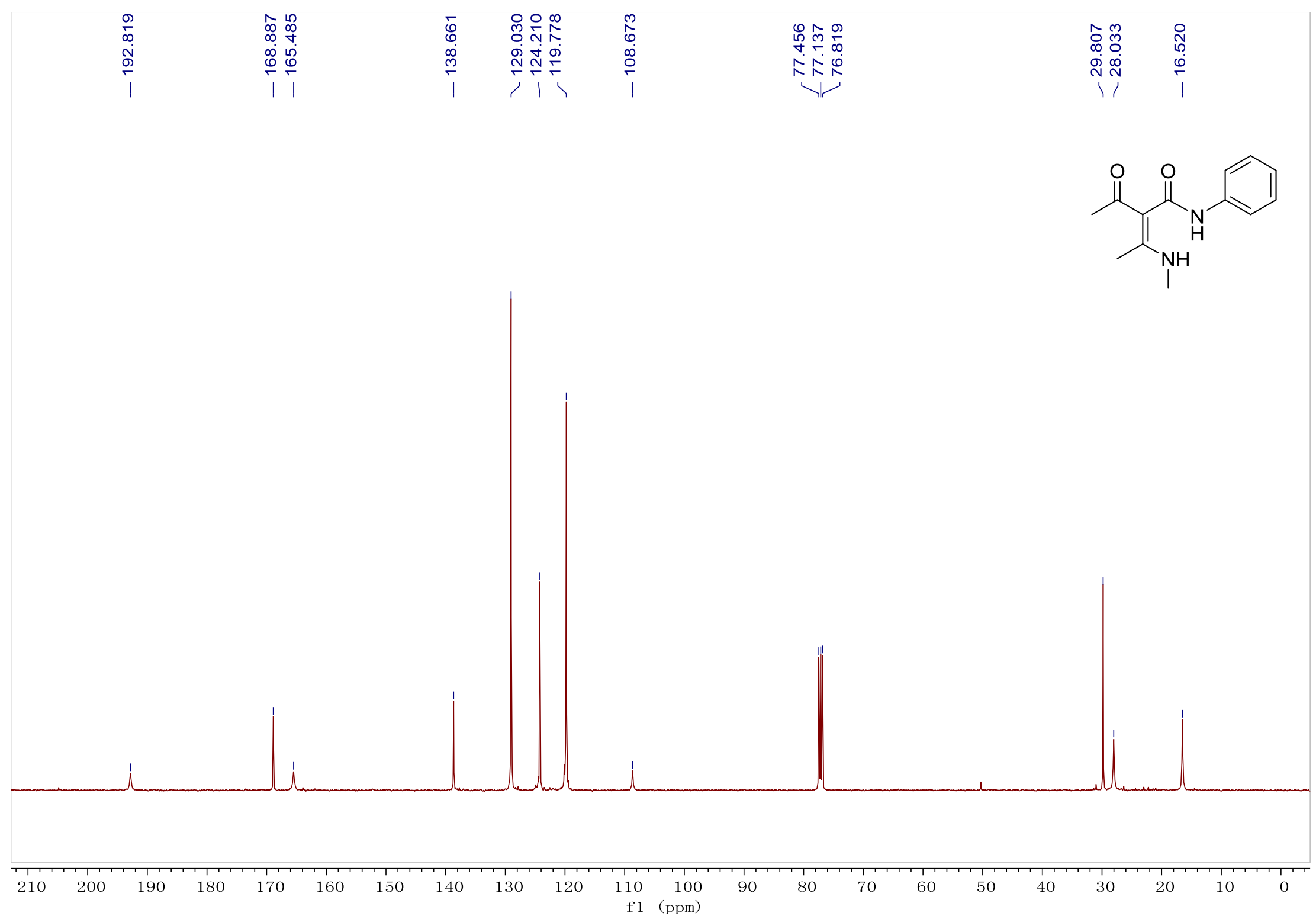


${ }^{1} \mathrm{H}$ NMR spectrum of $\mathbf{3 e}\left(300 \mathrm{MHz}, \mathrm{CDCl}_{3}\right)$

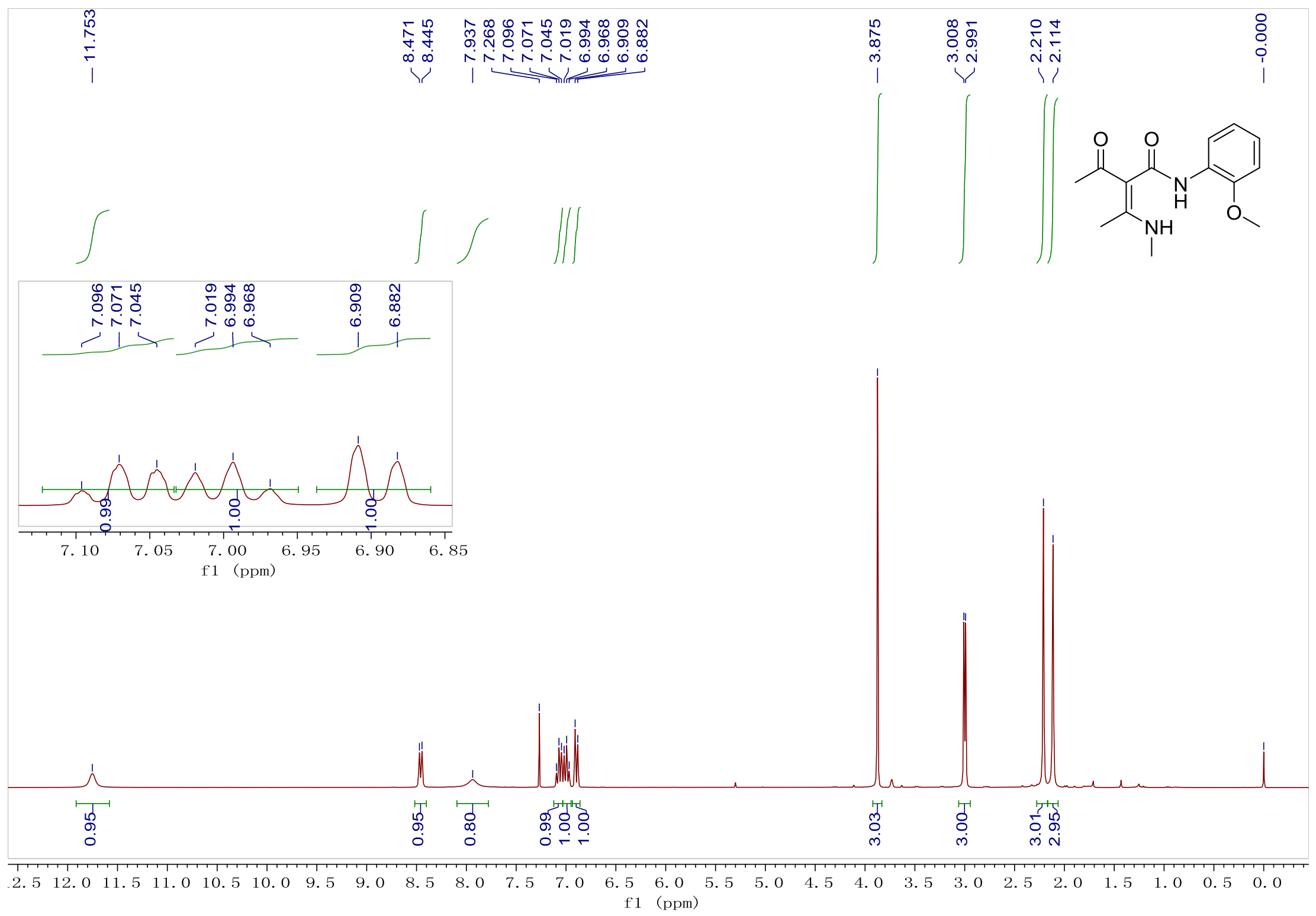

S21 
${ }^{13} \mathrm{C}\left\{{ }^{1} \mathrm{H}\right\}$ NMR spectrum of $\mathbf{3 e}\left(125 \mathrm{MHz}, \mathrm{CDCl}_{3}\right)$

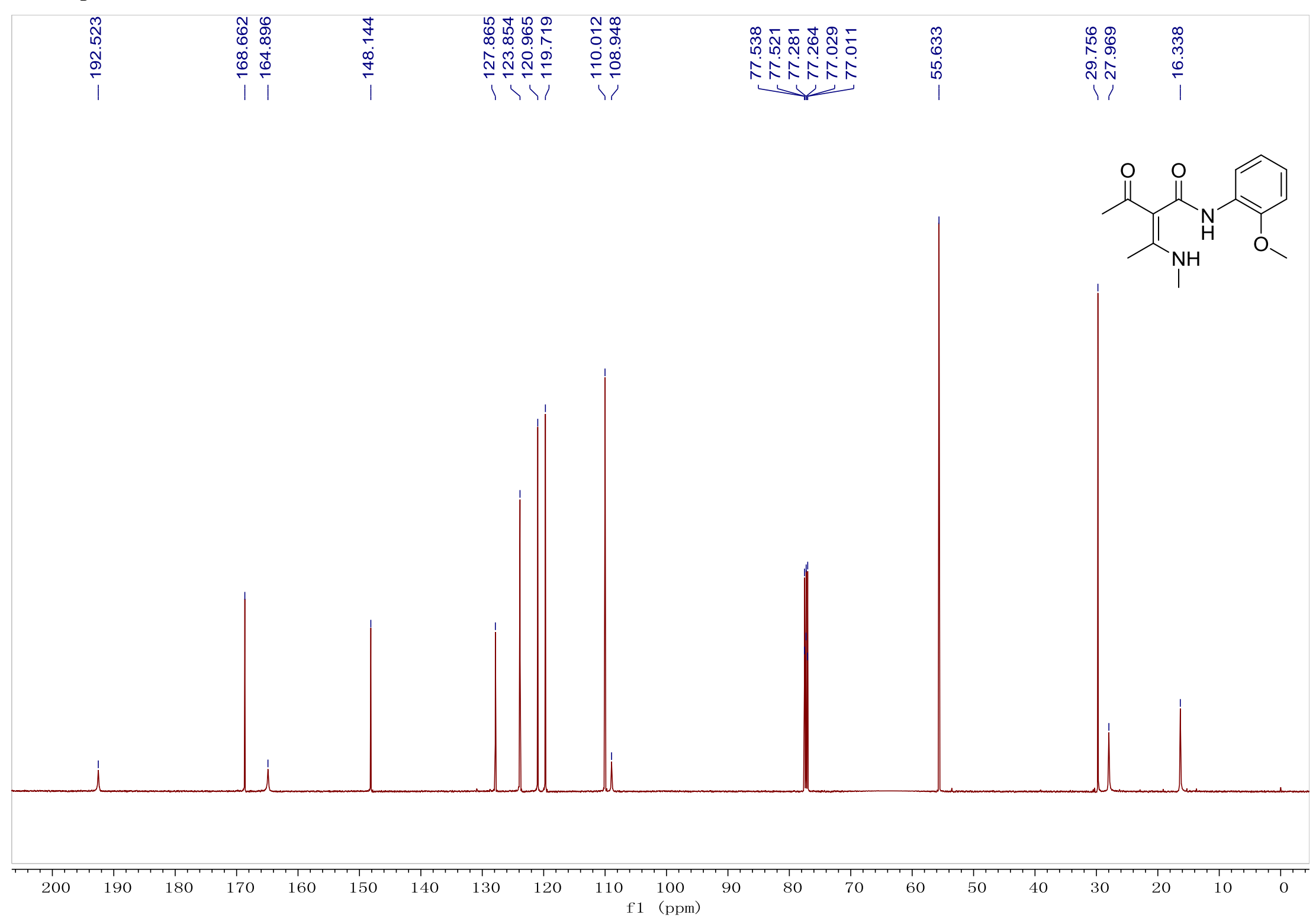


${ }^{1} \mathrm{H}$ NMR spectrum of $\mathbf{3 f}\left(300 \mathrm{MHz}, \mathrm{CDCl}_{3}\right)$

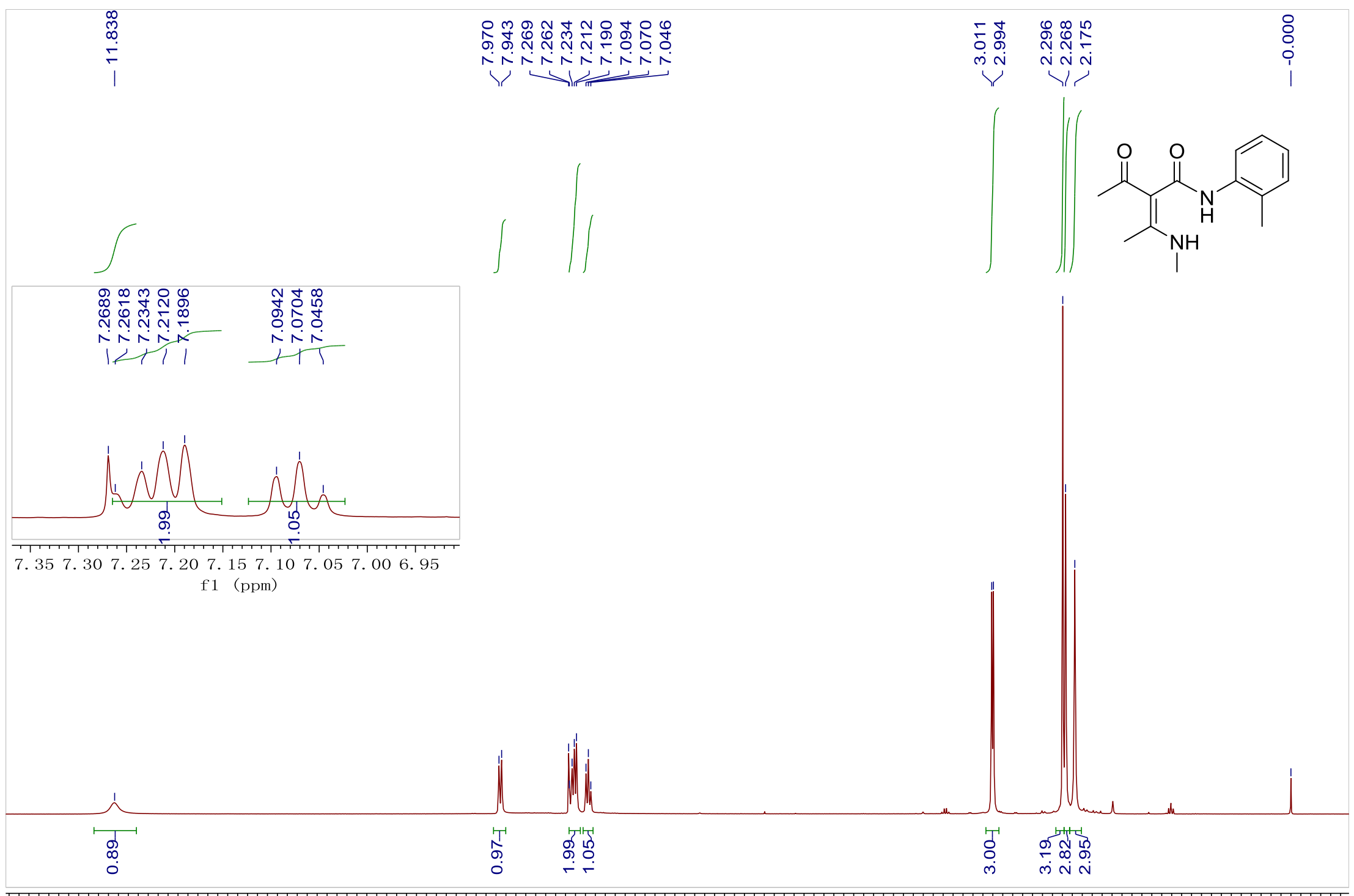

$\begin{array}{lllllllllllllllllllllllllllllllll}12.5 & 12.0 & 11.5 & 11.0 & 10.5 & 10.0 & 9.5 & 9.0 & 8.5 & 8.0 & 7.5 & 7.0 & 6.5 & 6.0 & 5.5 & 5.0 & 4.5 & 4.0 & 3.5 & 3.0 & 2.5 & 2.0 & 1.5 & 1.0 & 0.5 & 0.0 & -0\end{array}$ $\mathrm{f} 1 \quad(\mathrm{ppm})$ 
${ }^{13} \mathrm{C}\left\{{ }^{1} \mathrm{H}\right\}$ NMR spectrum of $\mathbf{3 f}\left(125 \mathrm{MHz}, \mathrm{CDCl}_{3}\right)$

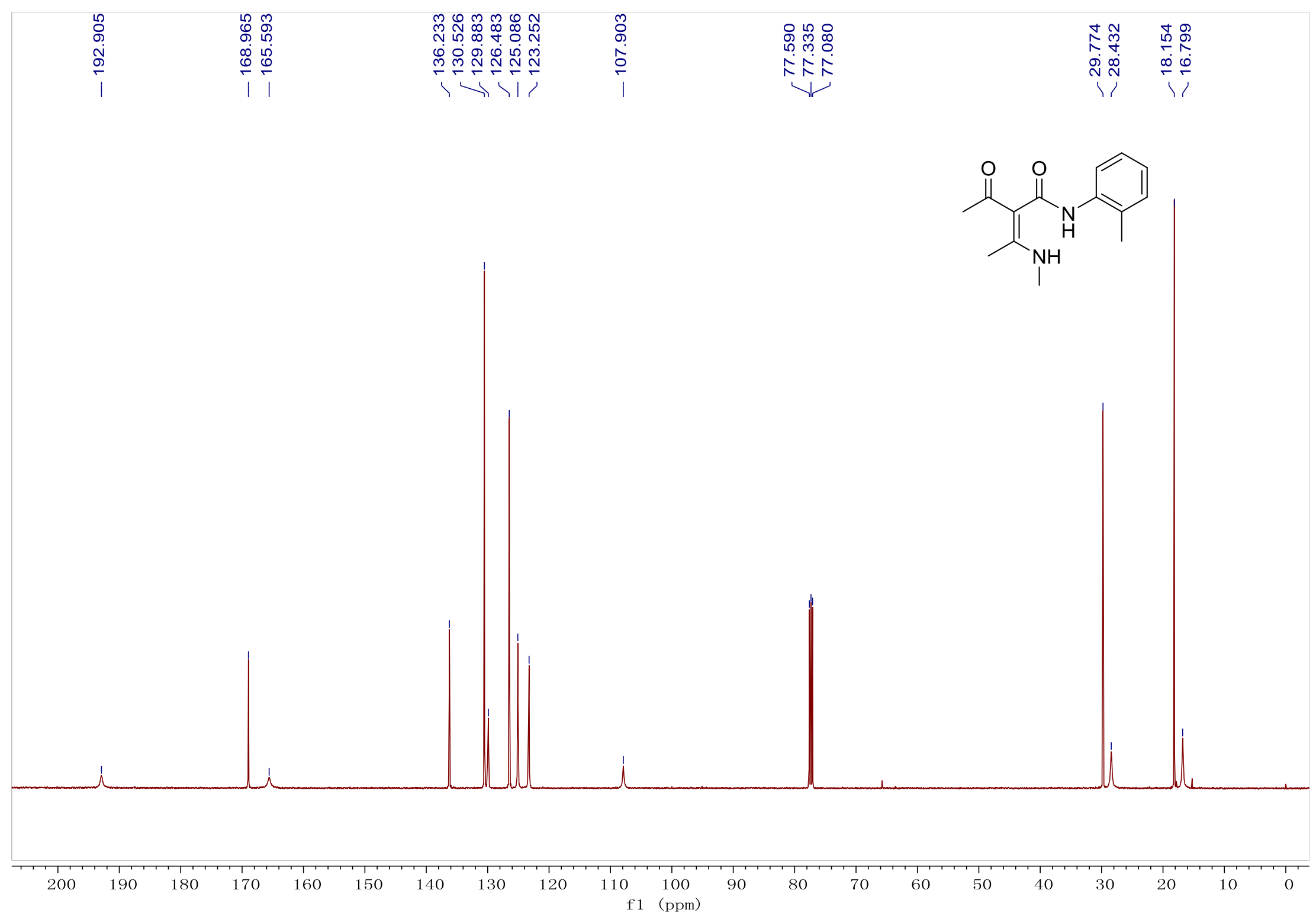


${ }^{1} \mathrm{H}$ NMR spectrum of $\mathbf{3 g}\left(300 \mathrm{MHz}, \mathrm{CDCl}_{3}\right)$

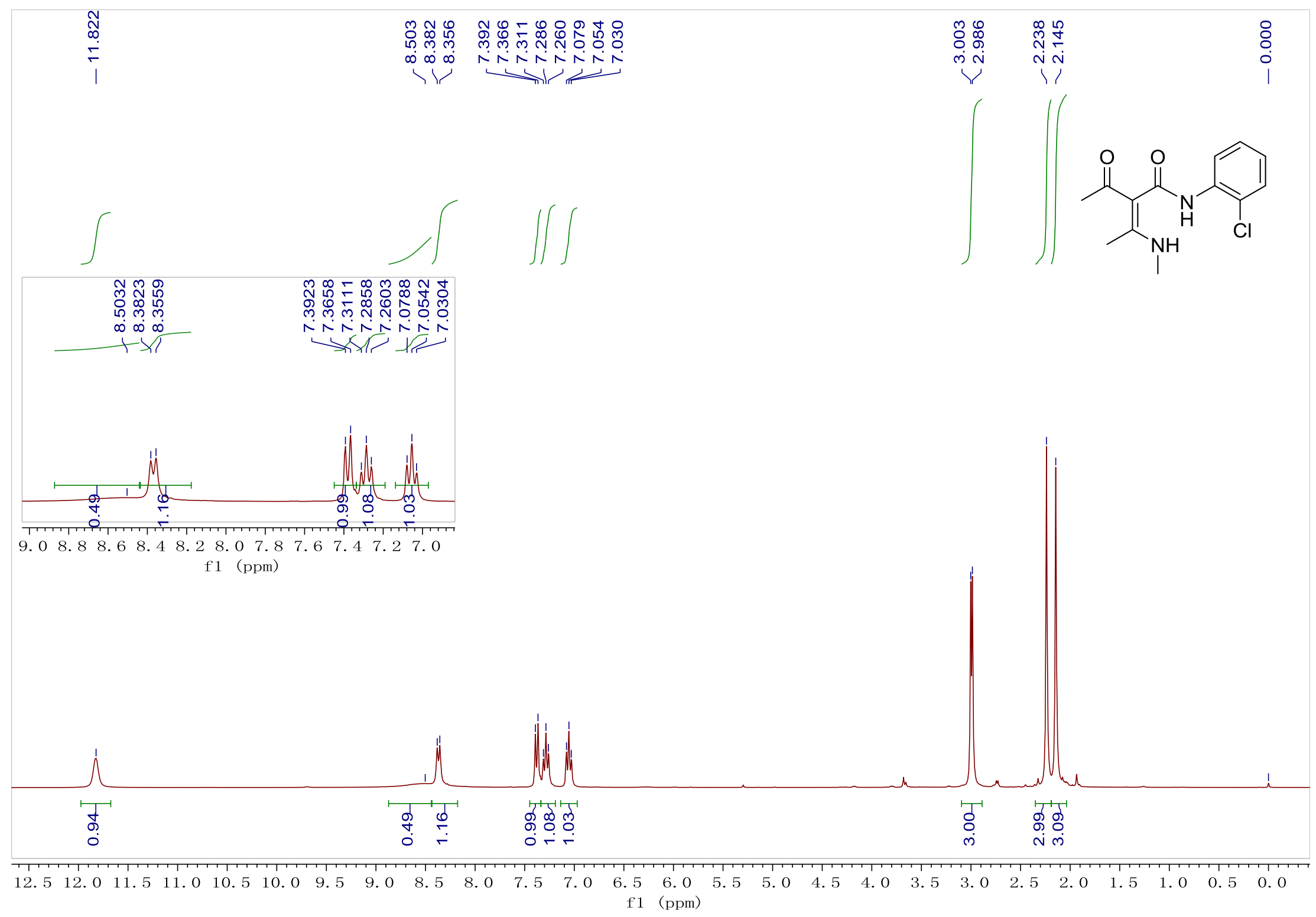

S25 
${ }^{13} \mathrm{C}\left\{{ }^{1} \mathrm{H}\right\}$ NMR spectrum of $\mathbf{3 g}\left(100 \mathrm{MHz}, \mathrm{CDCl}_{3}\right)$

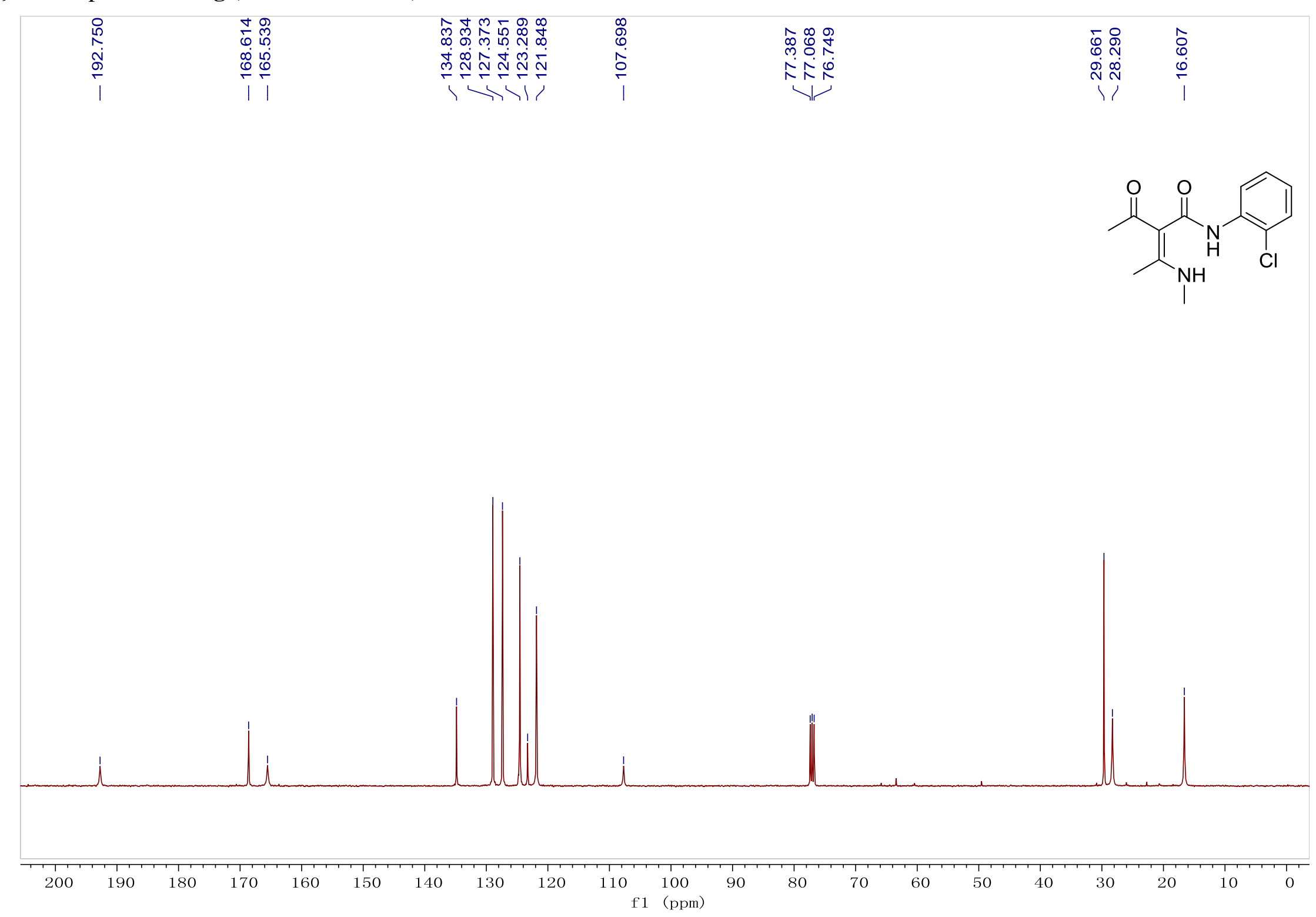


${ }^{1} \mathrm{H}$ NMR spectrum of $\mathbf{3 h}\left(300 \mathrm{MHz}, \mathrm{CDCl}_{3}\right)$

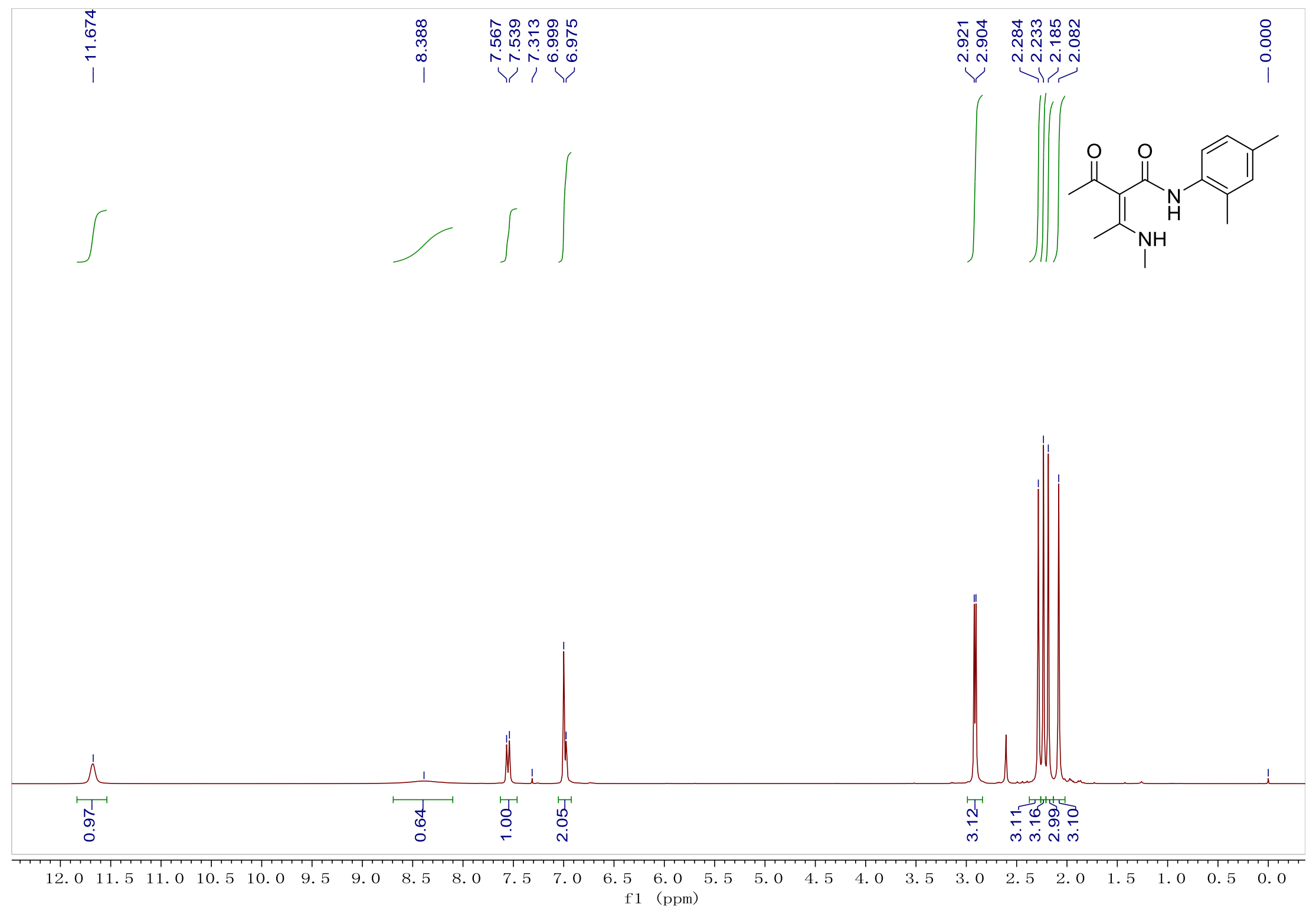


${ }^{13} \mathrm{C}\left\{{ }^{1} \mathrm{H}\right\}$ NMR spectrum of $\mathbf{3 h}\left(100 \mathrm{MHz}, \mathrm{CDCl}_{3}\right)$

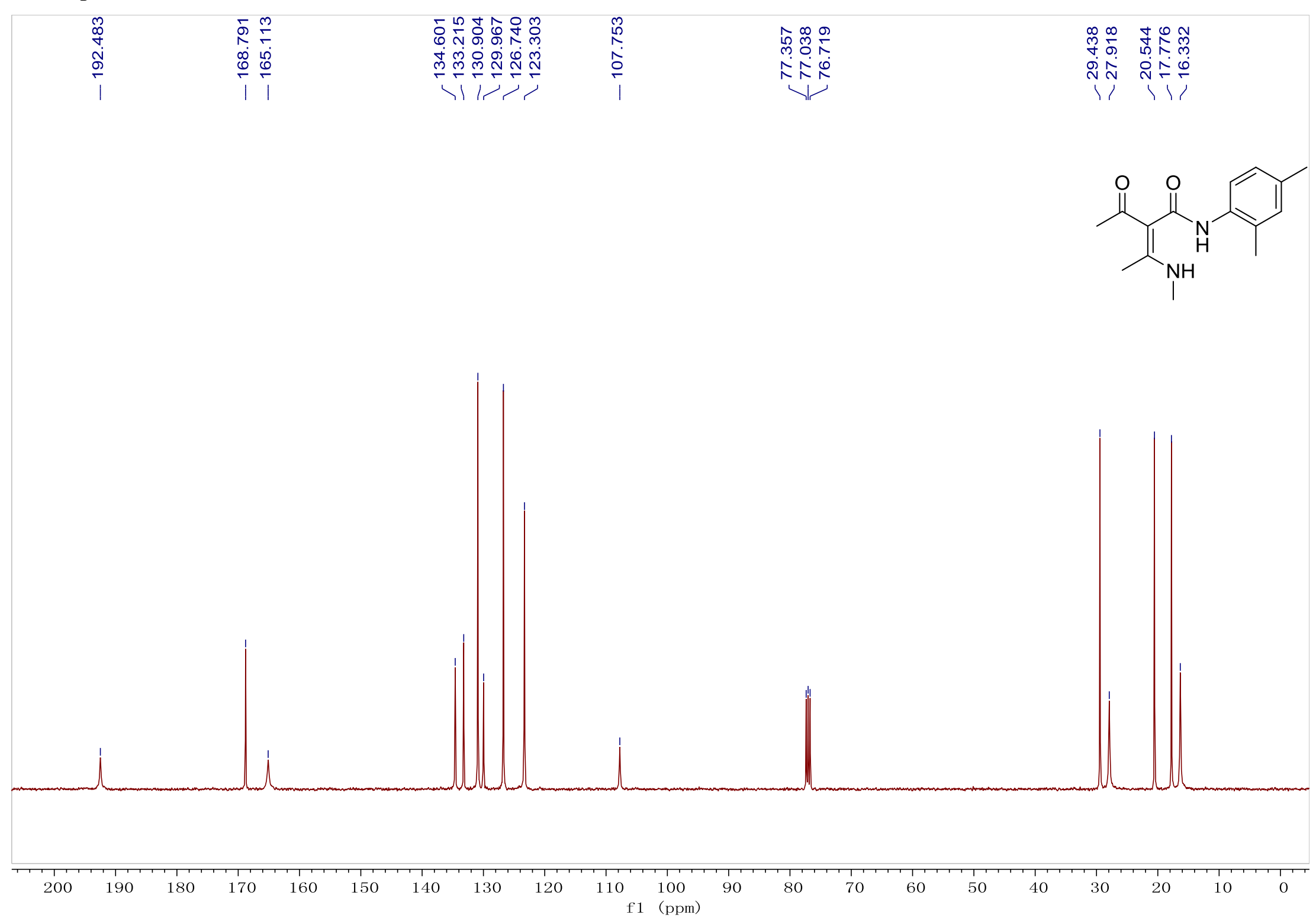


${ }^{1} \mathrm{H}$ NMR spectrum of $\mathbf{3 i}\left(300 \mathrm{MHz}, \mathrm{CDCl}_{3}\right)$

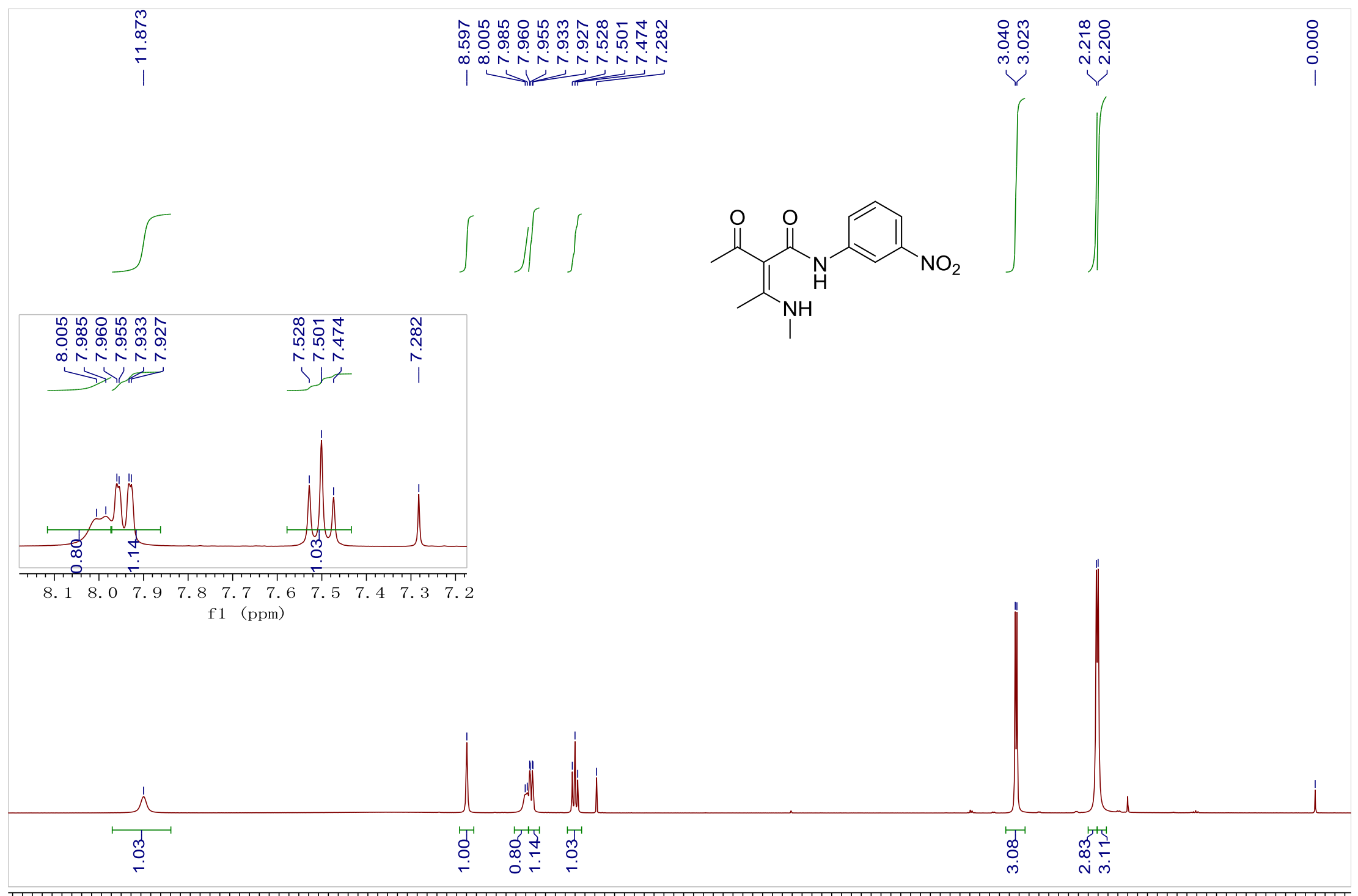

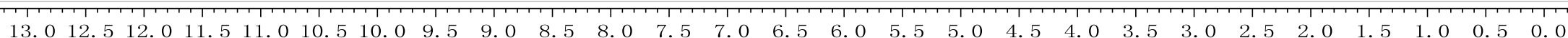
f1 (ppm) 
${ }^{13} \mathrm{C}\left\{{ }^{1} \mathrm{H}\right\}$ NMR spectrum of $3 \mathbf{i}\left(125 \mathrm{MHz}\right.$, DMSO-d $\left.\mathrm{d}_{6}\right)$

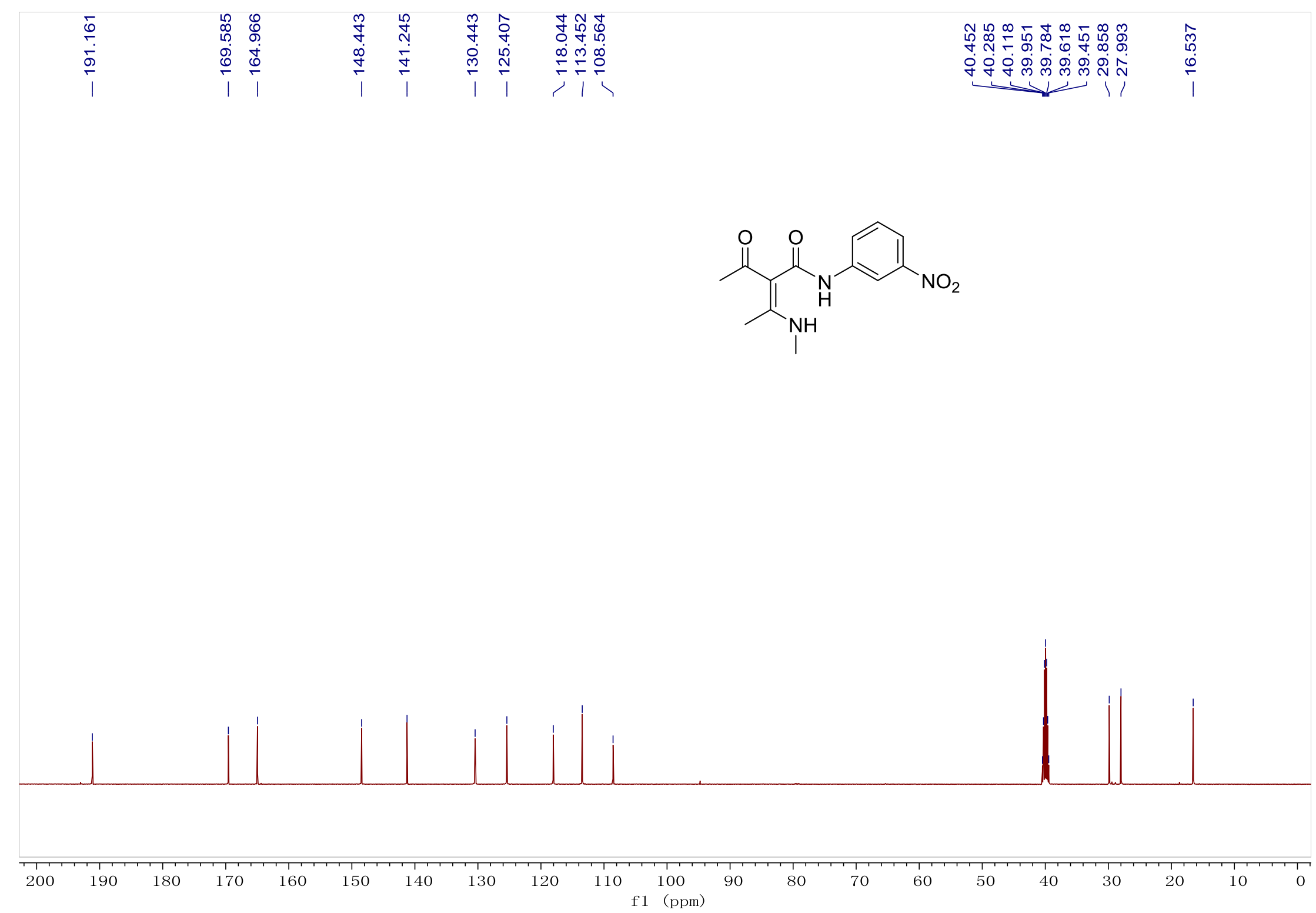


${ }^{1} \mathrm{H}$ NMR spectrum of $\mathbf{3 j}\left(400 \mathrm{MHz}, \mathrm{CDCl}_{3}\right.$ )

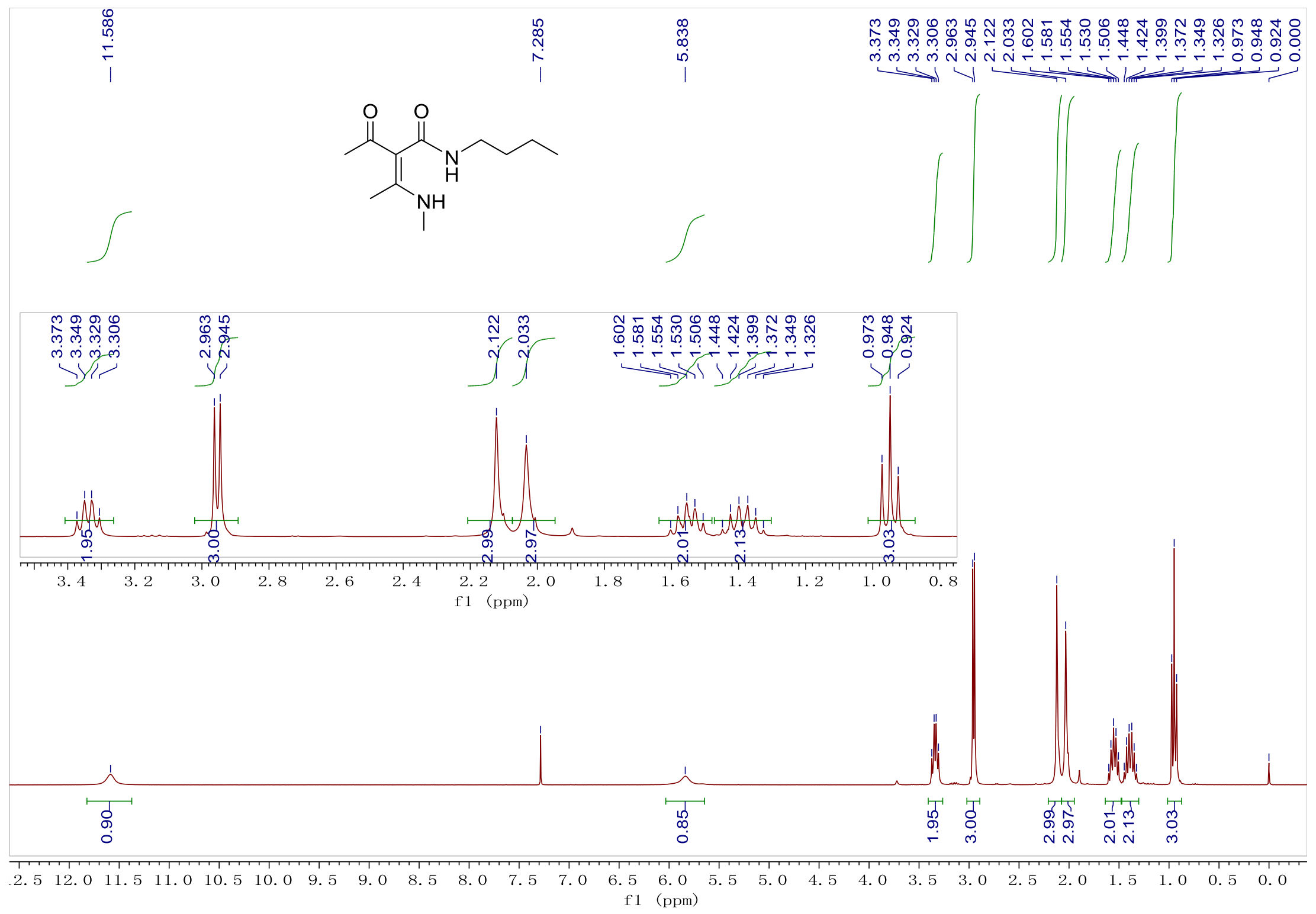

S31 
${ }^{13} \mathrm{C}\left\{{ }^{1} \mathrm{H}\right\}$ NMR spectrum of $\mathbf{3 j}\left(125 \mathrm{MHz}, \mathrm{CDCl}_{3}\right)$
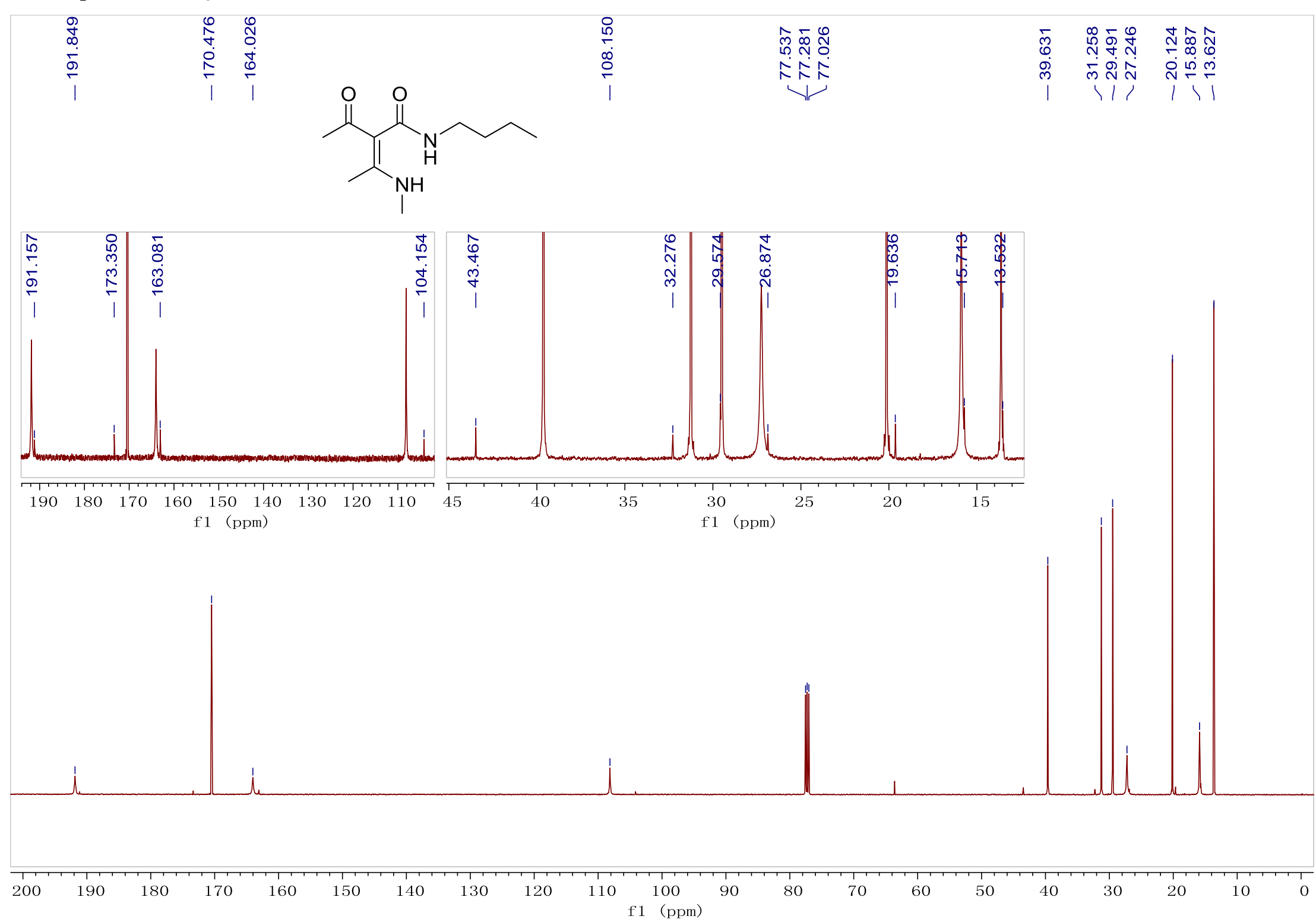
${ }^{1} \mathrm{H}$ NMR spectrum of $\mathbf{3 k}\left(300 \mathrm{MHz}, \mathrm{CDCl}_{3}\right.$ )

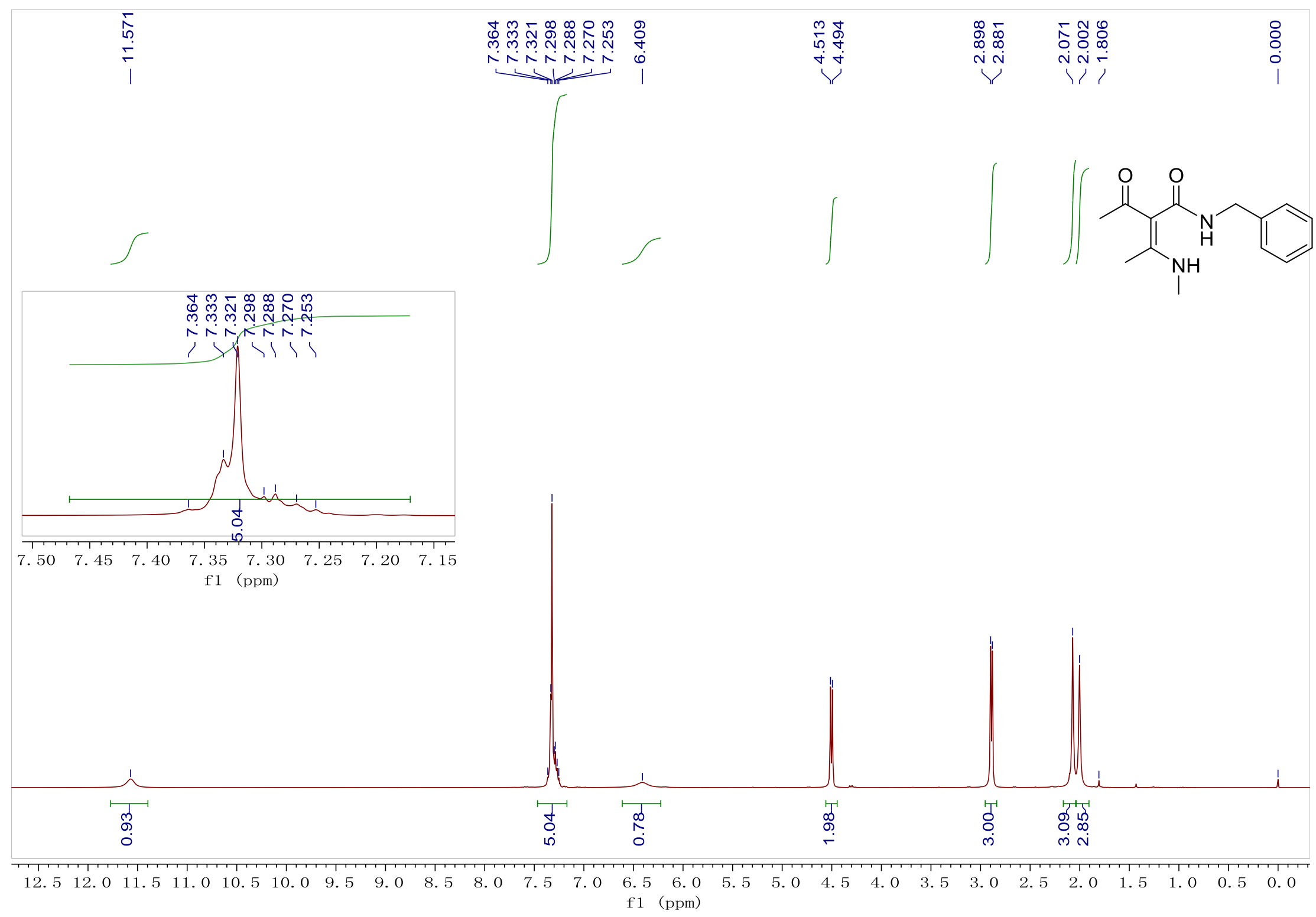

S33 
${ }^{13} \mathrm{C}\left\{{ }^{1} \mathrm{H}\right\}$ NMR spectrum of $3 \mathbf{k}\left(75 \mathrm{MHz}, \mathrm{CDCl}_{3}\right)$
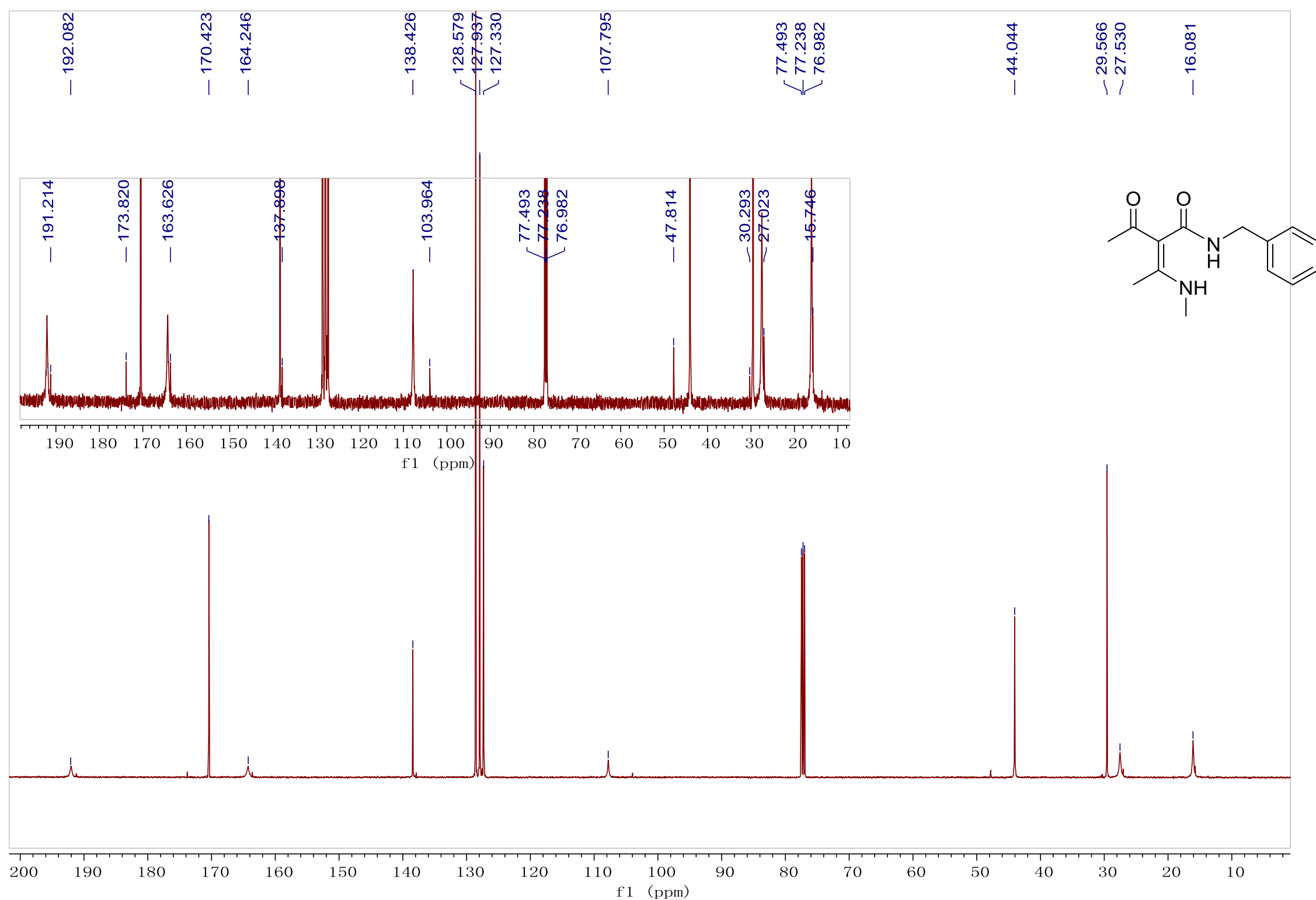
${ }^{1} \mathrm{H}$ NMR spectrum of $\mathbf{3 l}\left(300 \mathrm{MHz}, \mathrm{CDCl}_{3}\right)$

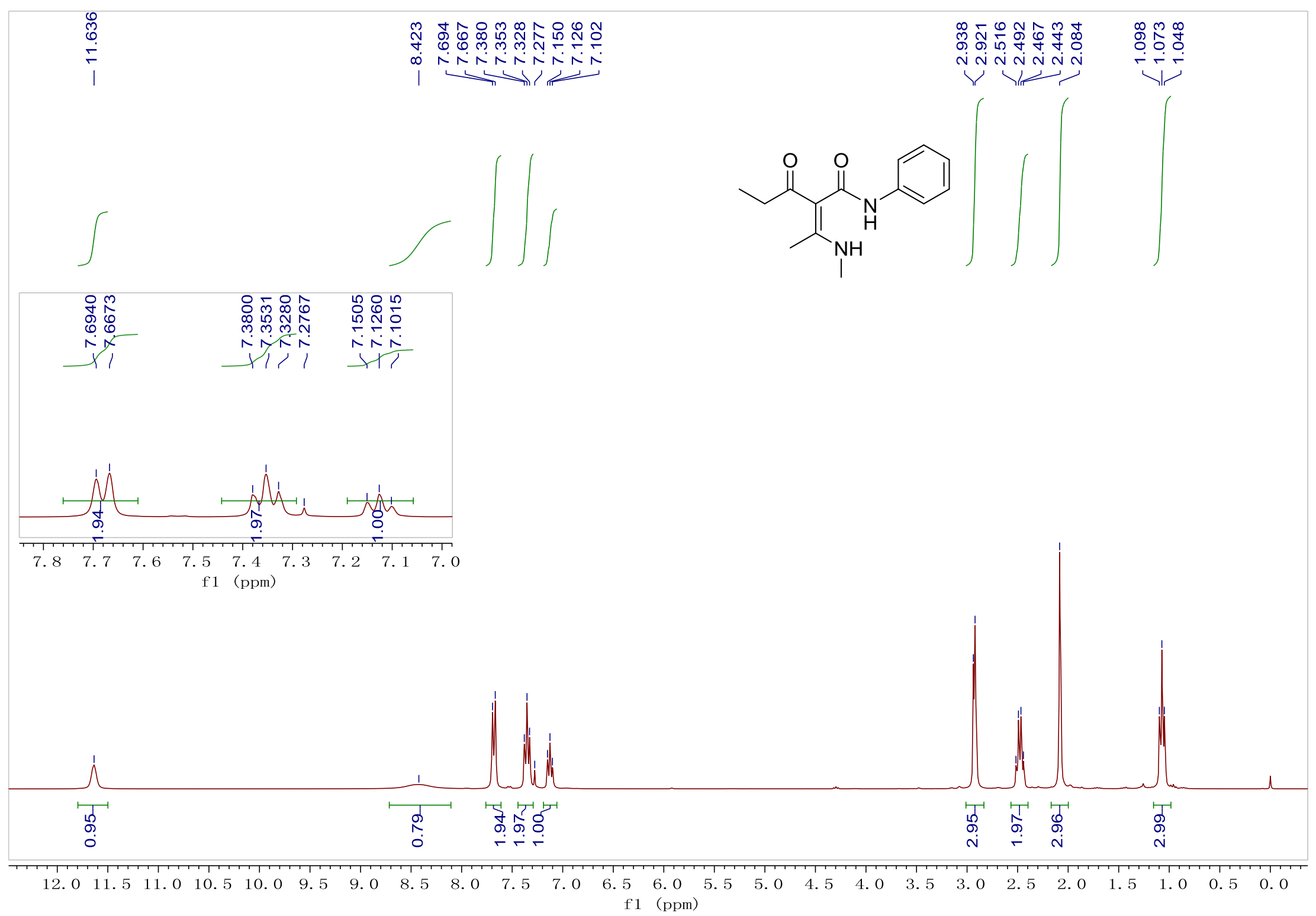

S35 
${ }^{13} \mathrm{C}\left\{{ }^{1} \mathrm{H}\right\}$ NMR spectrum of $31\left(100 \mathrm{MHz}, \mathrm{CDCl}_{3}\right)$

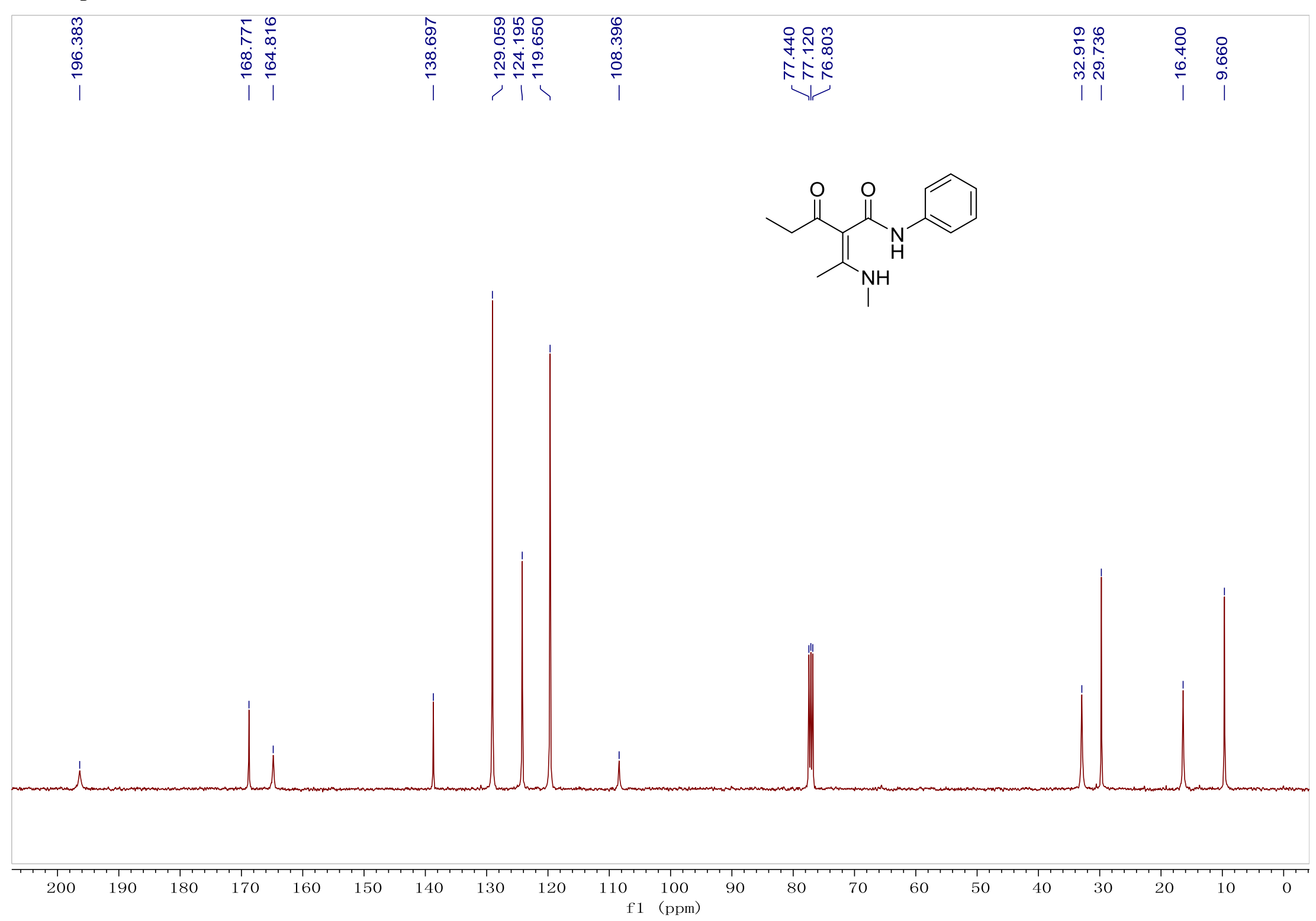


${ }^{1} \mathrm{H}$ NMR spectrum of $\mathbf{3 m}\left(300 \mathrm{MHz}, \mathrm{CDCl}_{3}\right)$

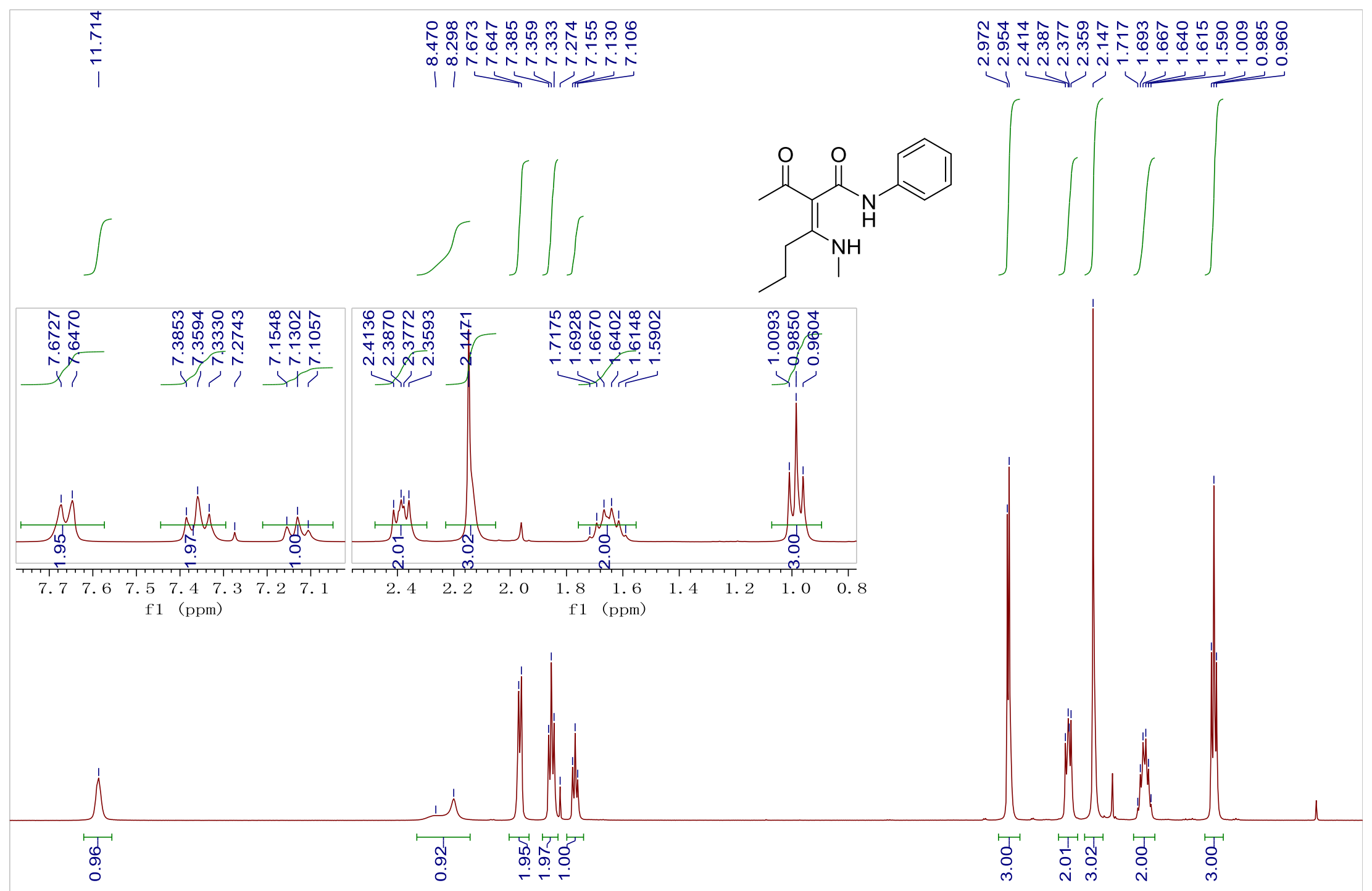

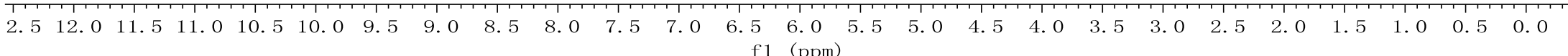


${ }^{13} \mathrm{C}\left\{{ }^{1} \mathrm{H}\right\}$ NMR spectrum of $\mathbf{3 m}\left(100 \mathrm{MHz}, \mathrm{CDCl}_{3}\right)$

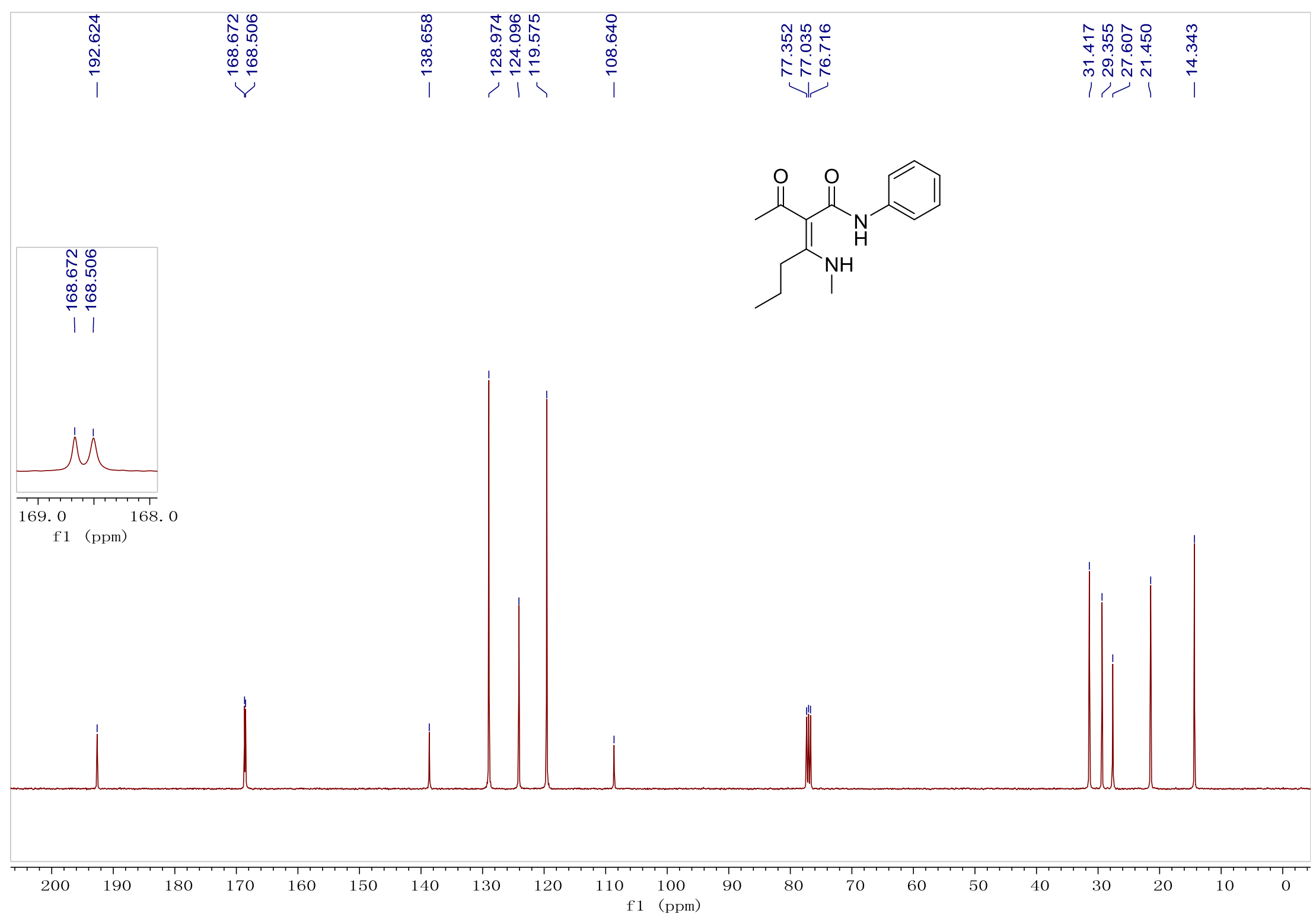


${ }^{1} \mathrm{H}$ NMR spectrum of 3 n (300 MHz, DMSO-d $\left.\mathrm{d}_{6}\right)$

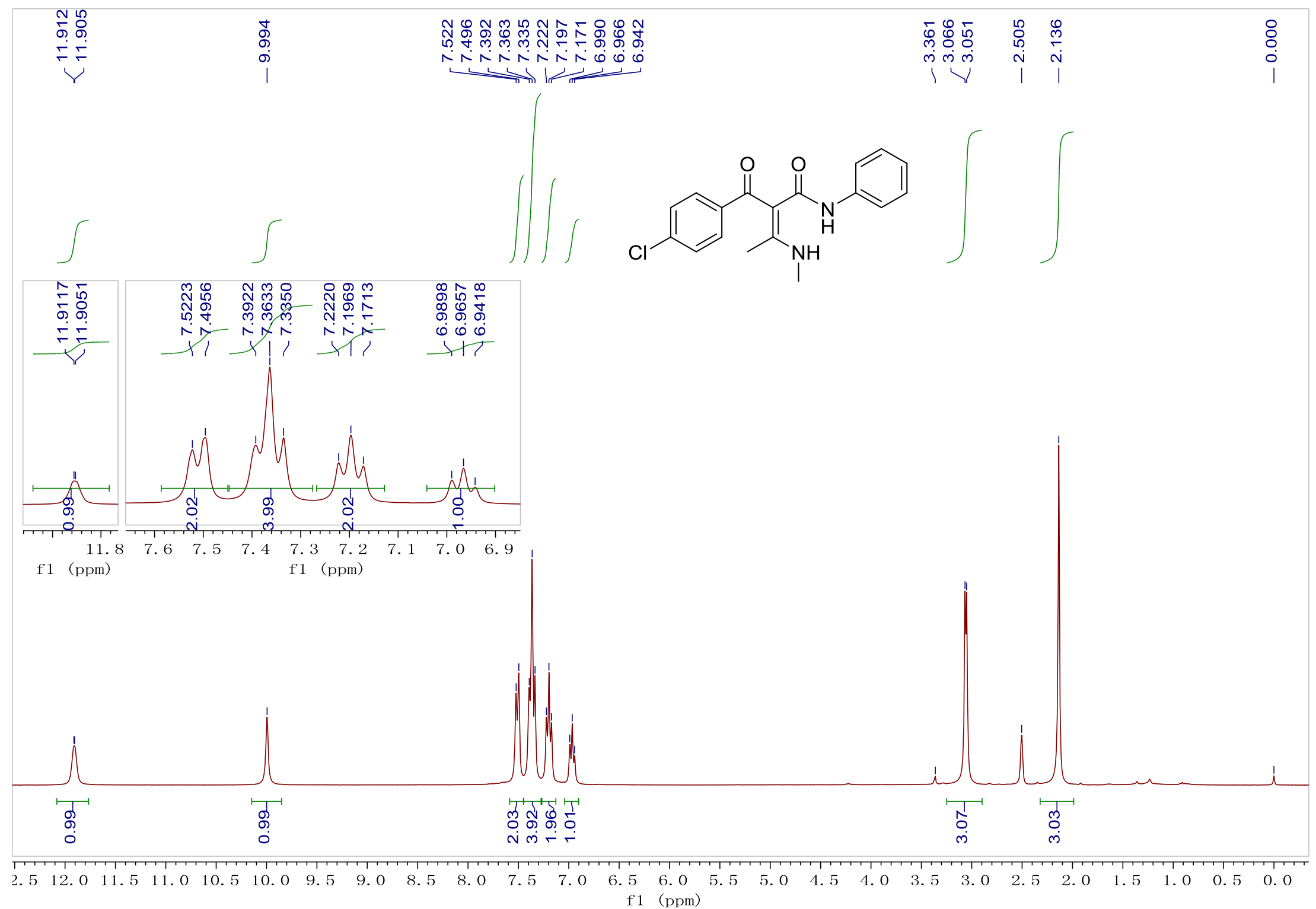


${ }^{13} \mathrm{C}\left\{{ }^{1} \mathrm{H}\right\}$ NMR spectrum of $3 \mathbf{n}\left(100 \mathrm{MHz}\right.$, DMSO-d $\left.\mathrm{d}_{6}\right)$
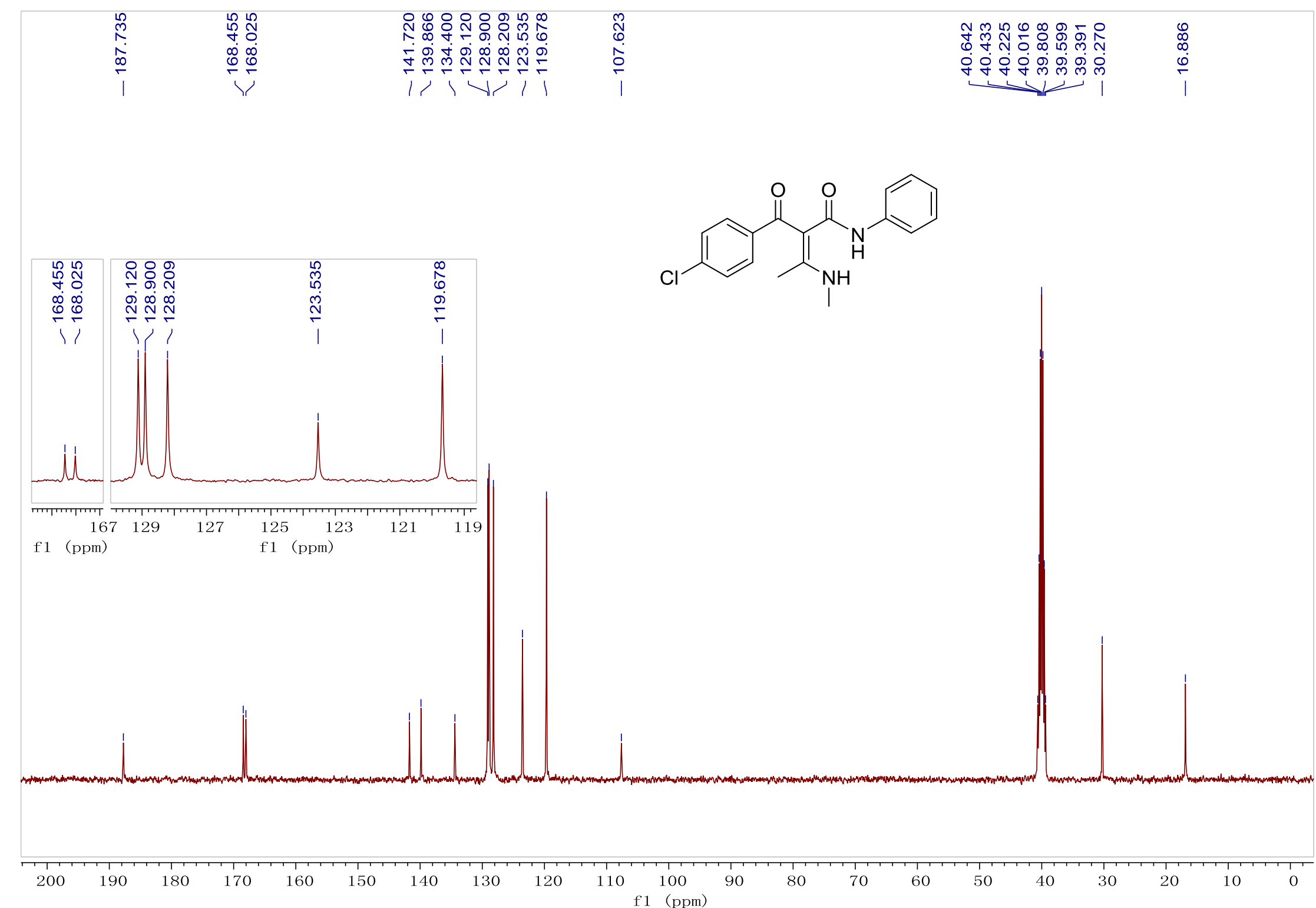

S40 
${ }^{1} \mathrm{H}$ NMR spectrum of $\mathbf{3 o}\left(300 \mathrm{MHz}, \mathrm{CDCl}_{3}\right)$

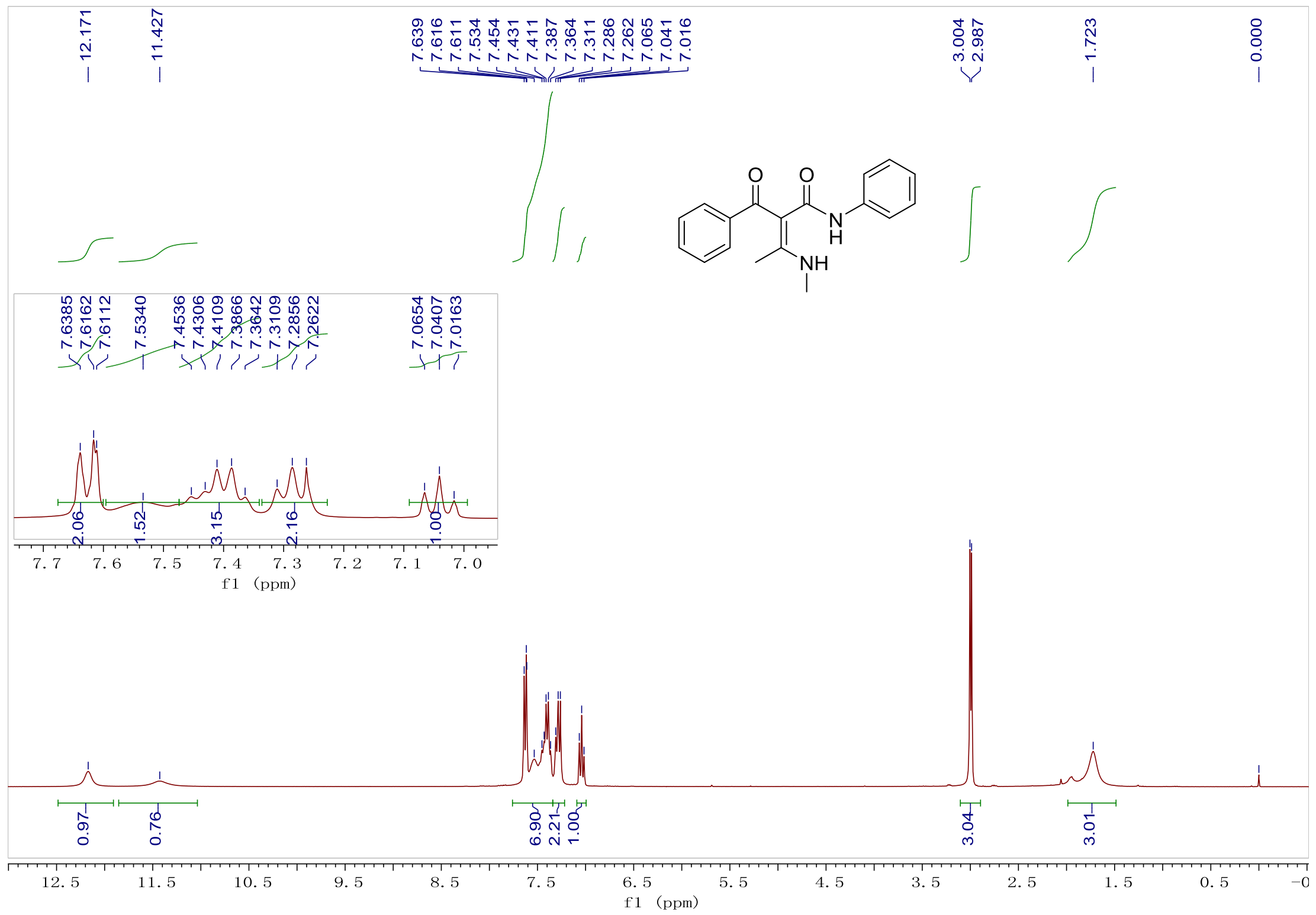

S41 
${ }^{13} \mathrm{C}\left\{{ }^{1} \mathrm{H}\right\}$ NMR spectrum of $30\left(100 \mathrm{MHz}, \mathrm{CDCl}_{3}\right)$
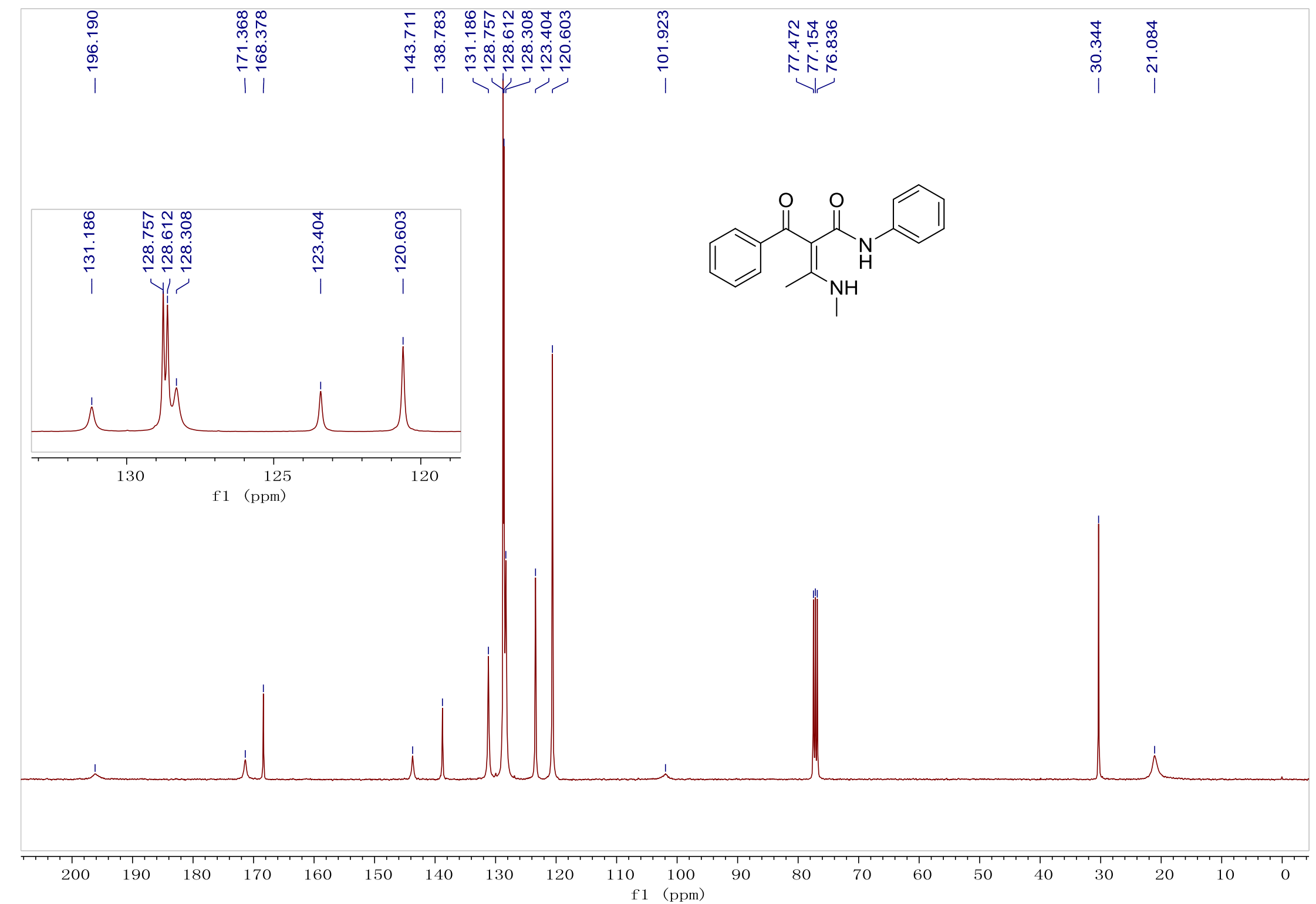
${ }^{1} \mathrm{H}$ NMR spectrum of $\mathbf{4 a}\left(300 \mathrm{MHz}, \mathrm{CDCl}_{3}\right)$

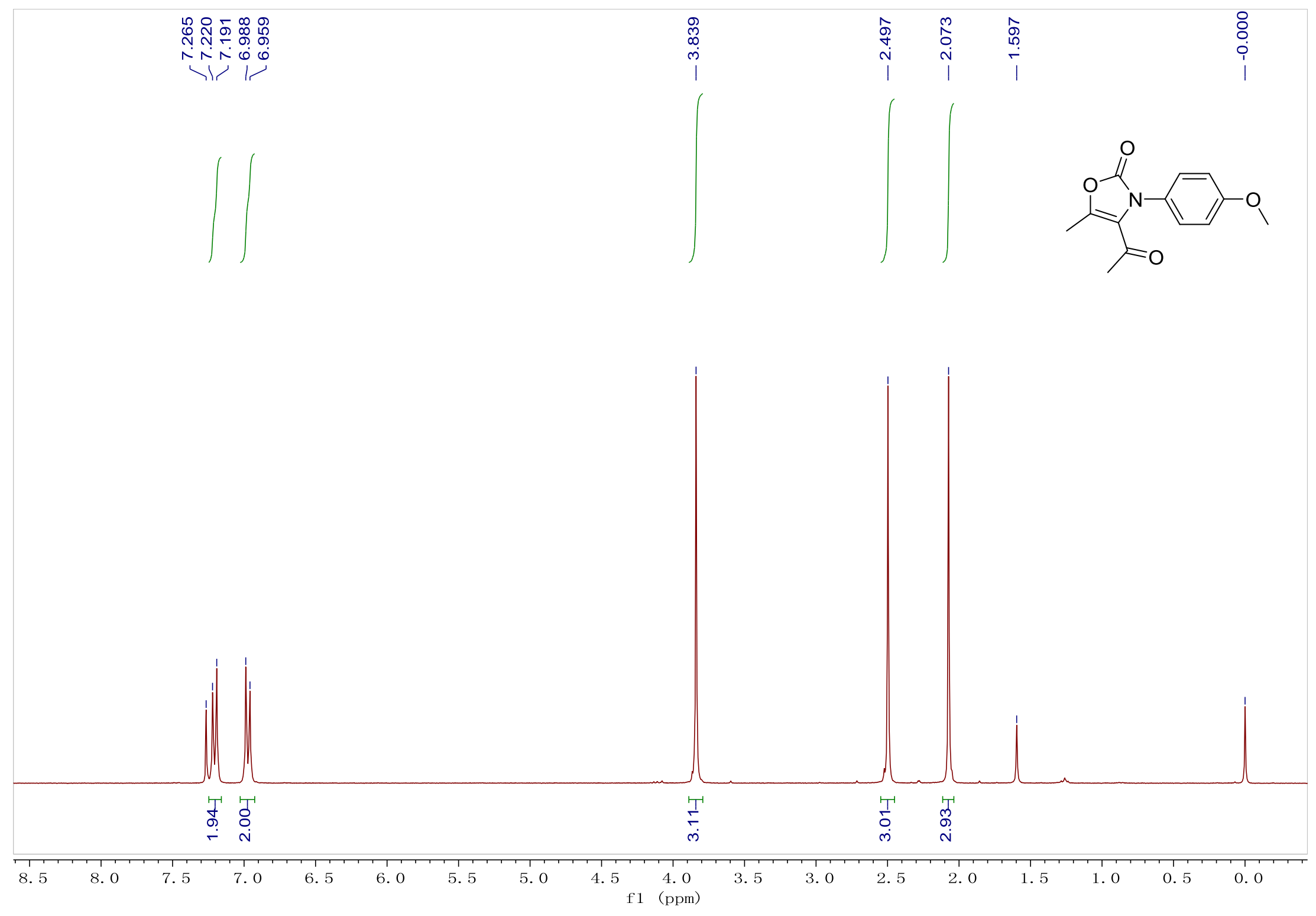


${ }^{13} \mathrm{C}\left\{{ }^{1} \mathrm{H}\right\}$ NMR spectrum of $\mathbf{4 a}\left(100 \mathrm{MHz}, \mathrm{CDCl}_{3}\right)$
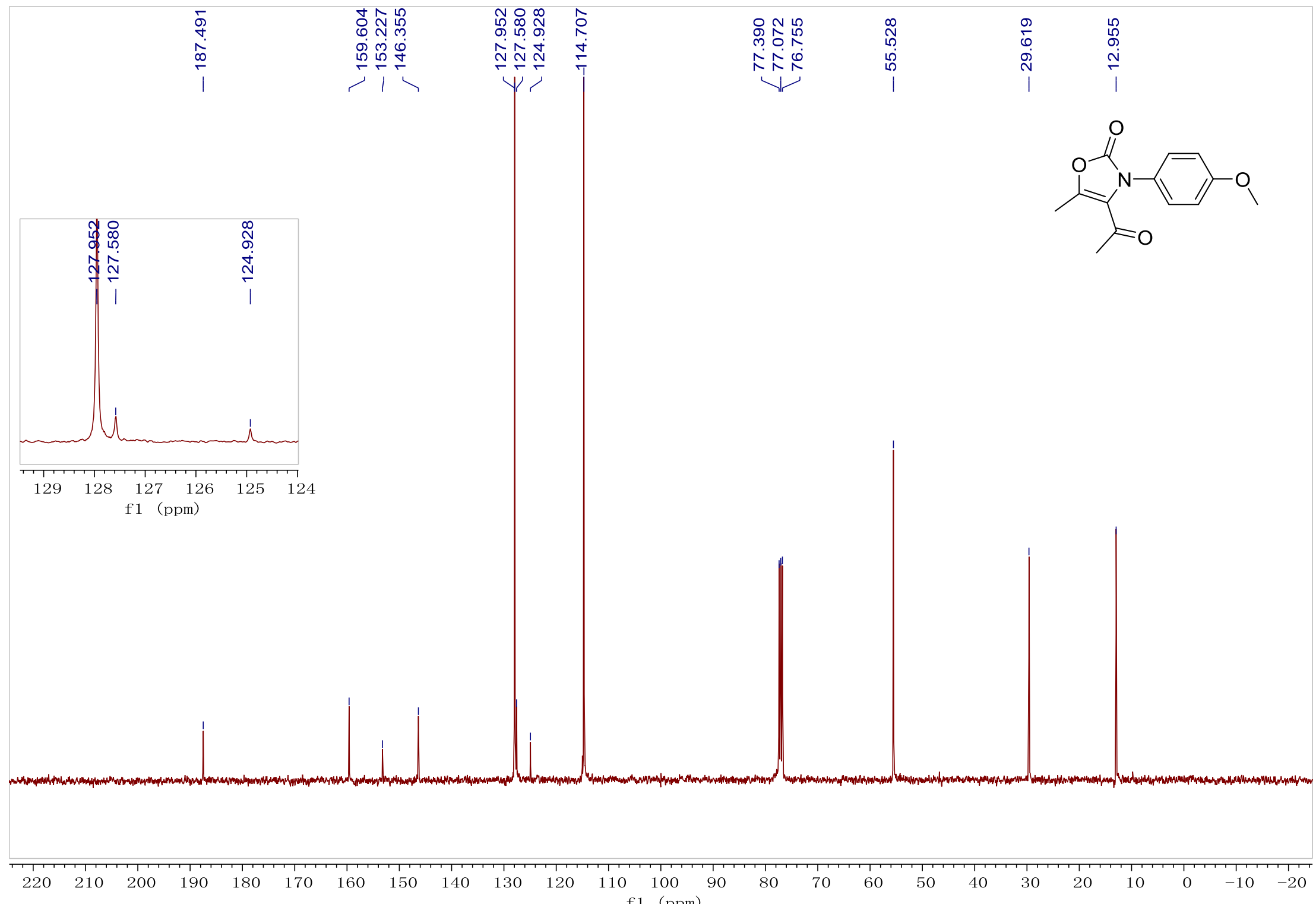
${ }^{1} \mathrm{H}$ NMR spectrum of $\mathbf{4 b}\left(300 \mathrm{MHz}, \mathrm{CDCl}_{3}\right)$

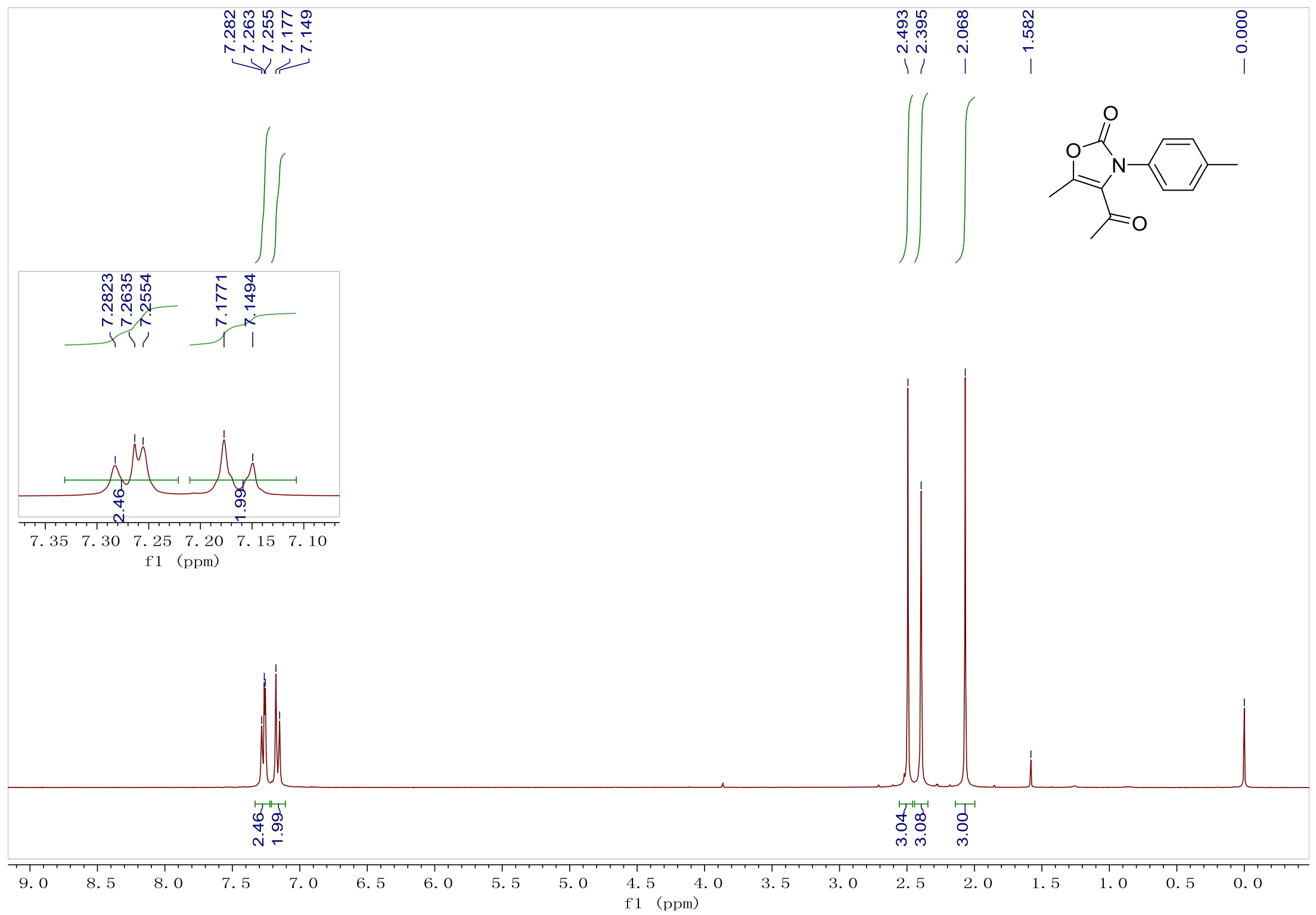

S45 
${ }^{13} \mathrm{C}\left\{{ }^{1} \mathrm{H}\right\}$ NMR spectrum of $\mathbf{4 b}\left(100 \mathrm{MHz}, \mathrm{CDCl}_{3}\right)$

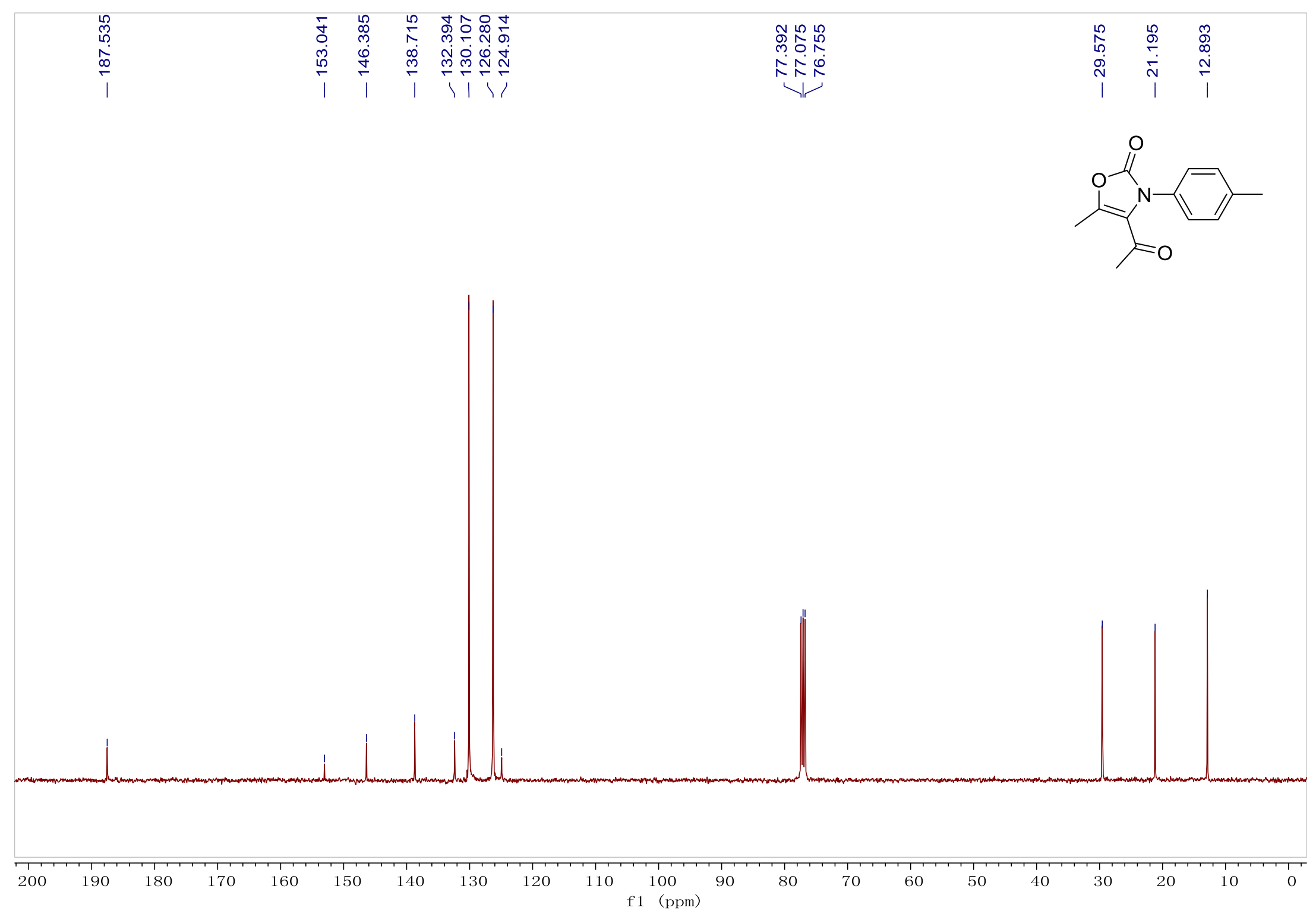


${ }^{1} \mathrm{H}$ NMR spectrum of $\mathbf{4 c}\left(400 \mathrm{MHz}, \mathrm{CDCl}_{3}\right)$

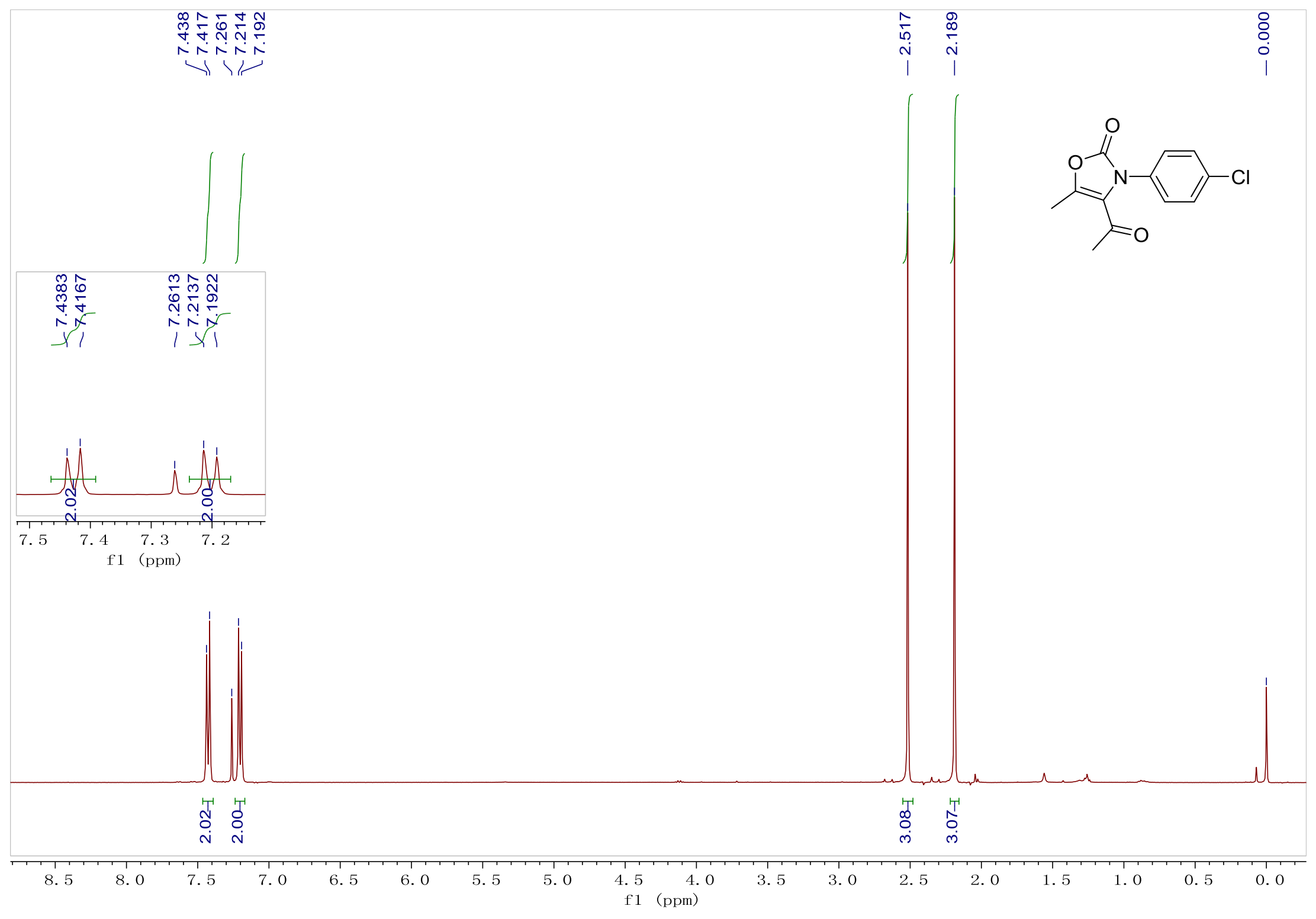

S47 
${ }^{13} \mathrm{C}\left\{{ }^{1} \mathrm{H}\right\}$ NMR spectrum of $\mathbf{4 c}\left(100 \mathrm{MHz}, \mathrm{CDCl}_{3}\right)$

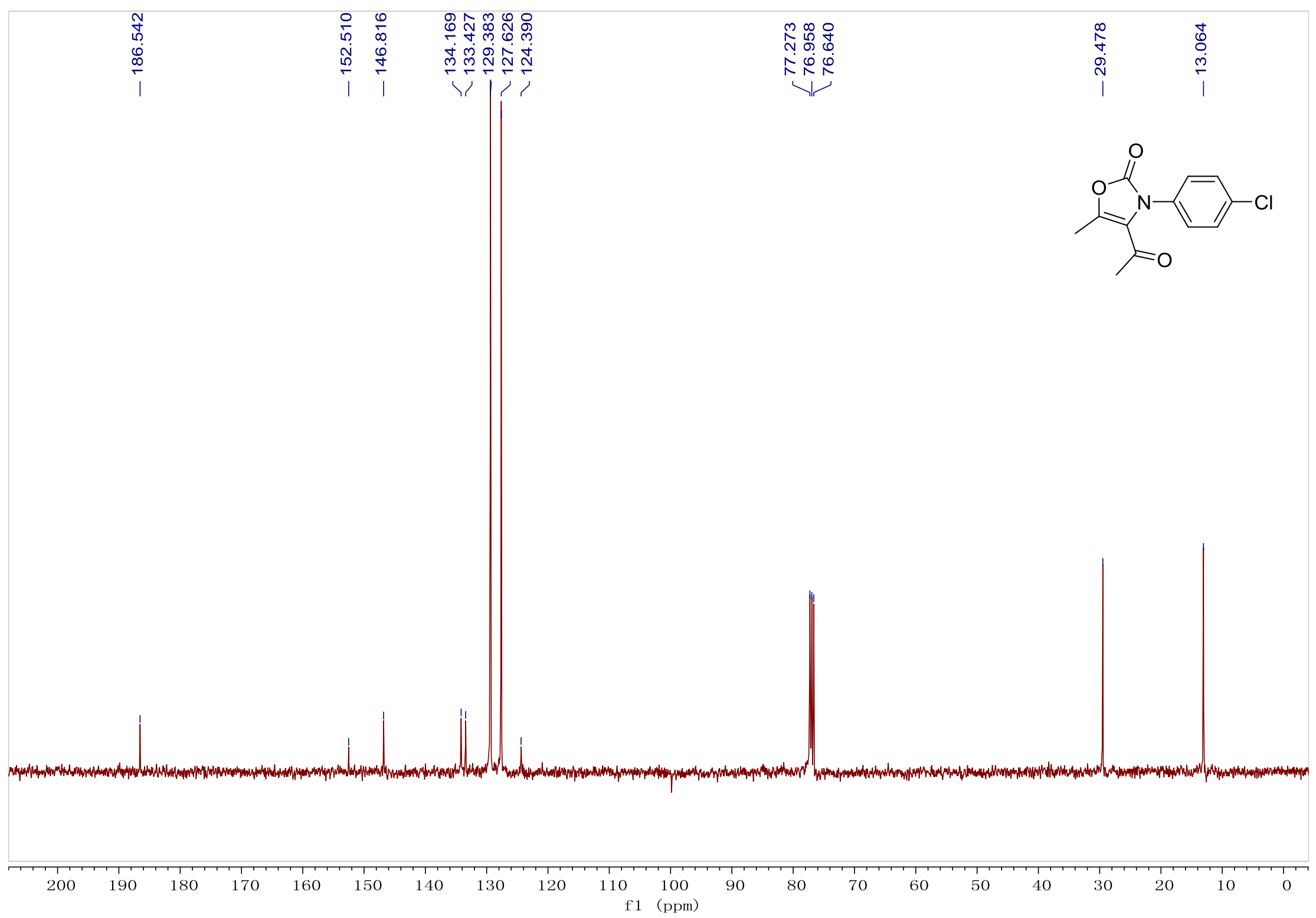


${ }^{1} \mathrm{H}$ NMR spectrum of $\mathbf{4 d}\left(300 \mathrm{MHz}, \mathrm{CDCl}_{3}\right)$

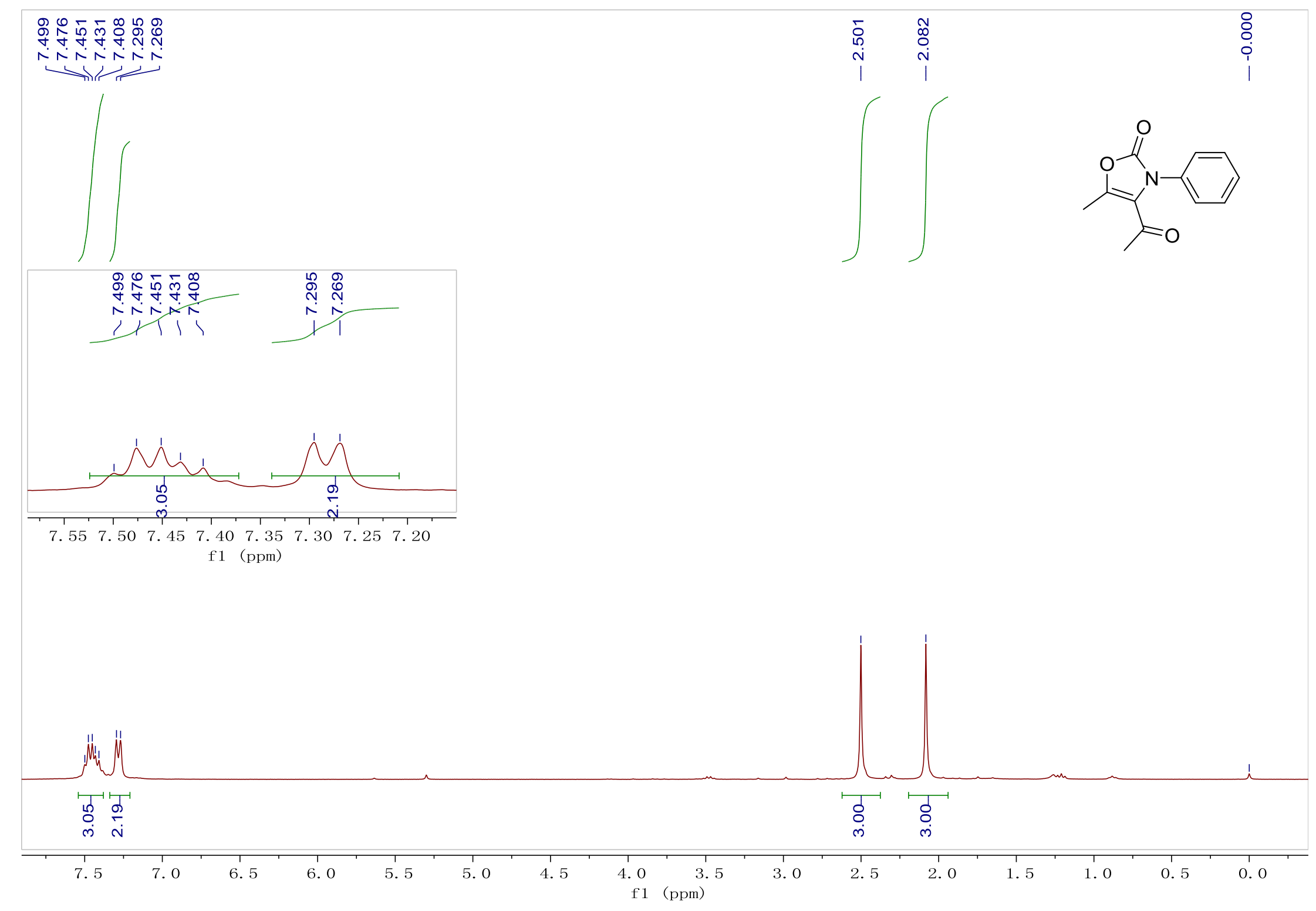


${ }^{13} \mathrm{C}\left\{{ }^{1} \mathrm{H}\right\}$ NMR spectrum of $\mathbf{4 d}\left(75 \mathrm{MHz}, \mathrm{CDCl}_{3}\right)$

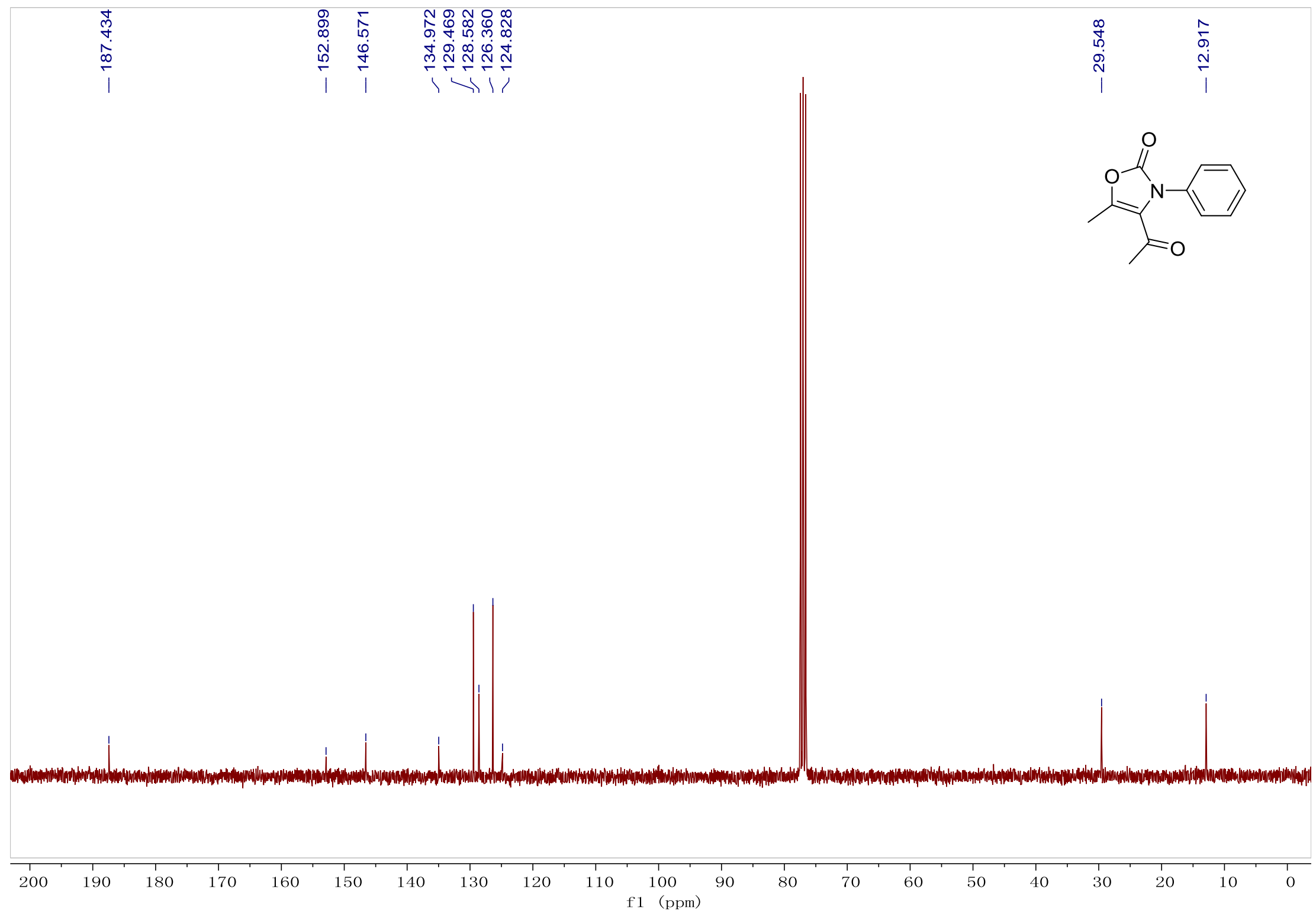


${ }^{1} \mathrm{H}$ NMR spectrum of $\mathbf{4 e}\left(300 \mathrm{MHz}, \mathrm{CDCl}_{3}\right)$

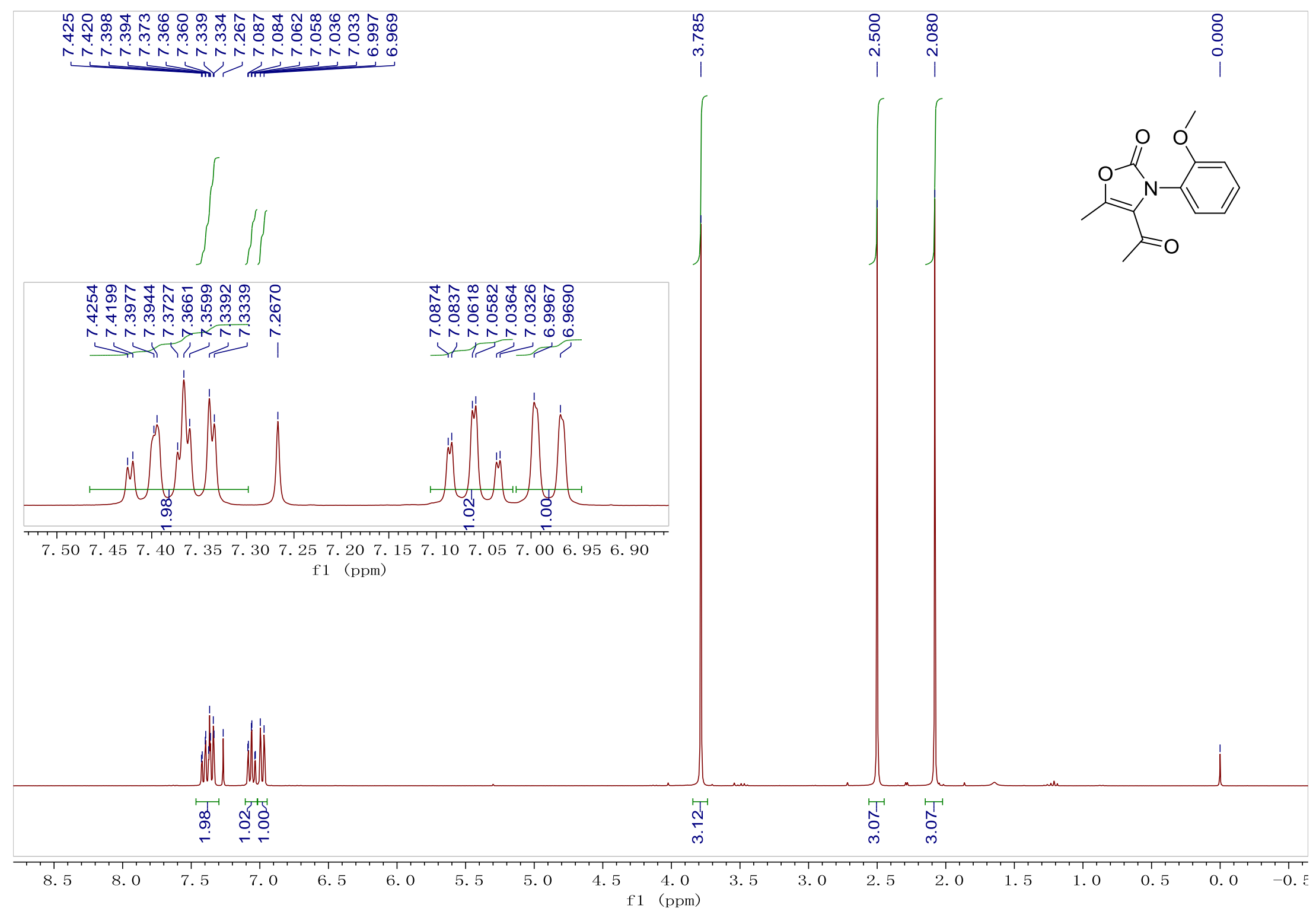

S51 
${ }^{13} \mathrm{C}\left\{{ }^{1} \mathrm{H}\right\}$ NMR spectrum of $4 \mathbf{e}\left(75 \mathrm{MHz}, \mathrm{CDCl}_{3}\right)$
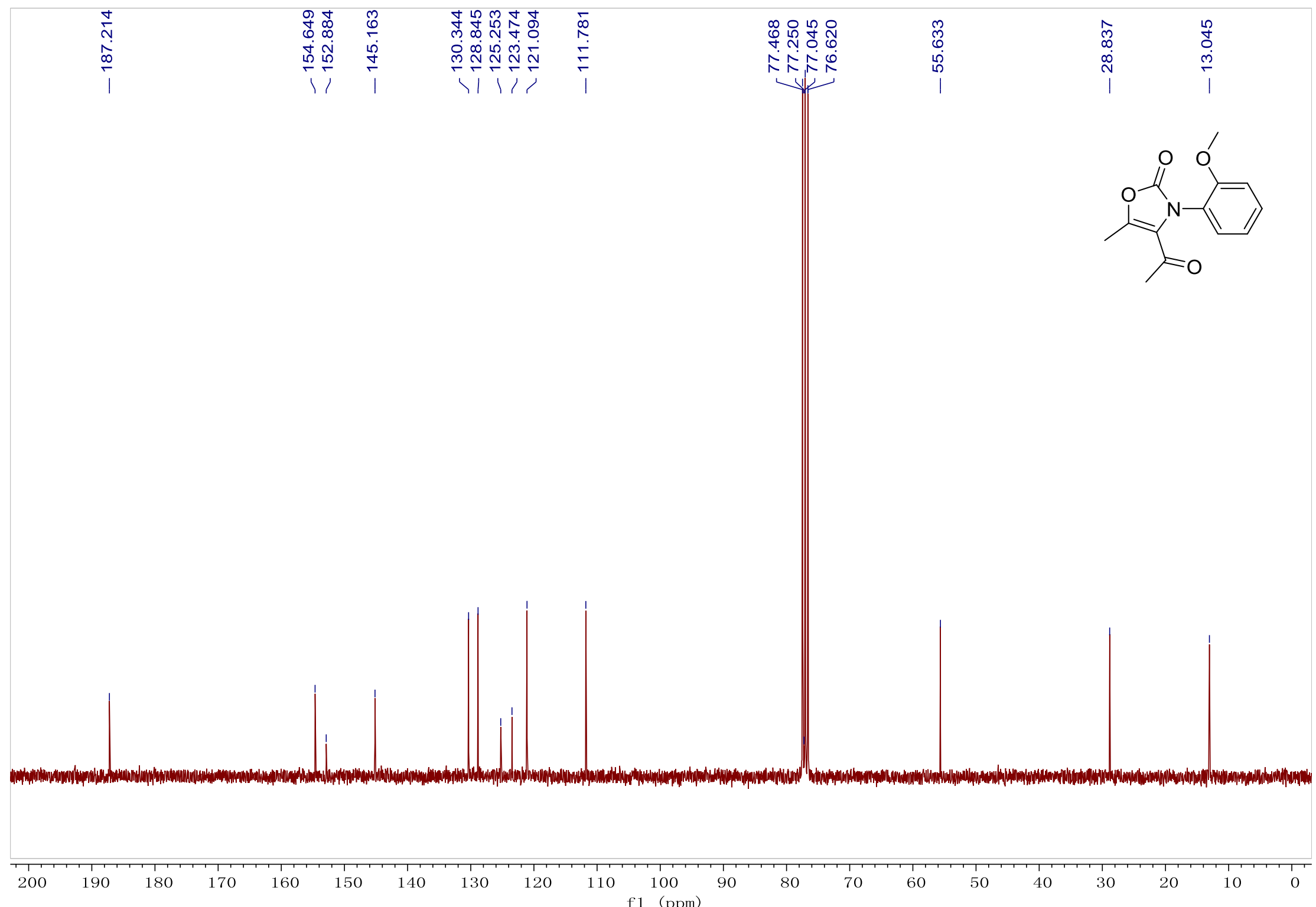
${ }^{1} \mathrm{H}$ NMR spectrum of $\mathbf{4 f}\left(300 \mathrm{MHz}, \mathrm{CDCl}_{3}\right)$

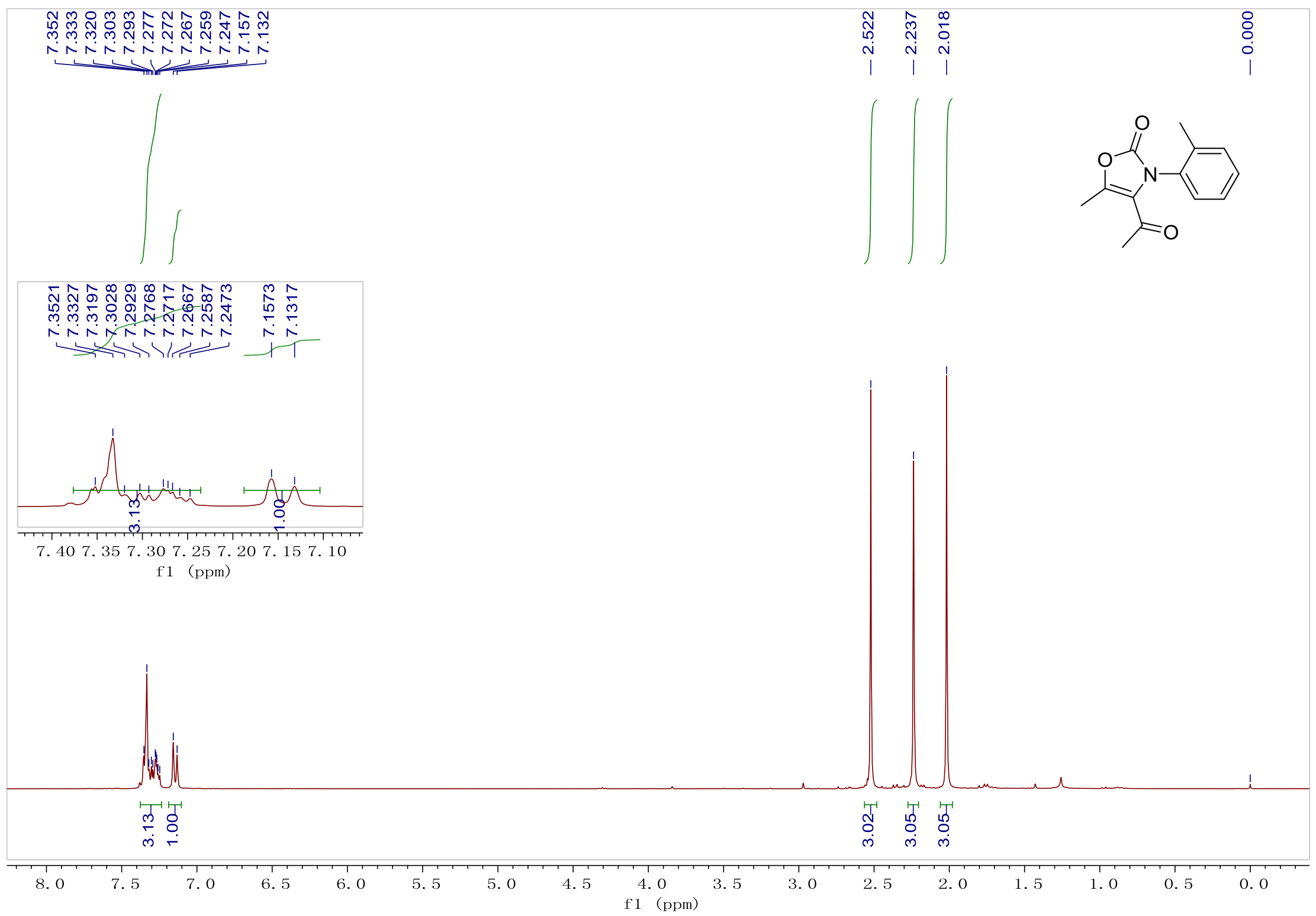


${ }^{13} \mathrm{C}\left\{{ }^{1} \mathrm{H}\right\}$ NMR spectrum of $\mathbf{4 f}\left(75 \mathrm{MHz}, \mathrm{CDCl}_{3}\right)$
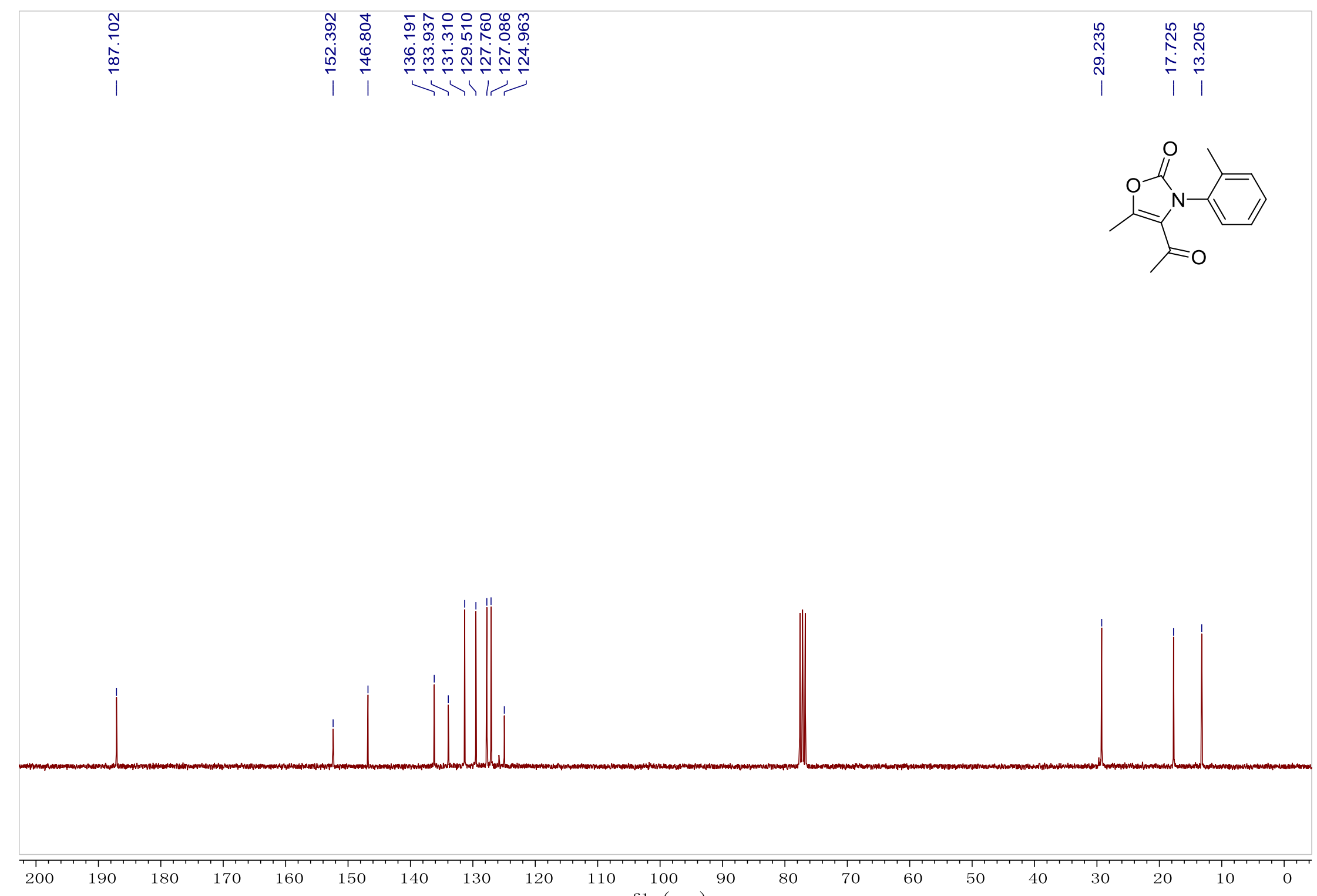
${ }^{1} \mathrm{H}$ NMR spectrum of $\mathbf{4 g}\left(300 \mathrm{MHz}, \mathrm{CDCl}_{3}\right)$

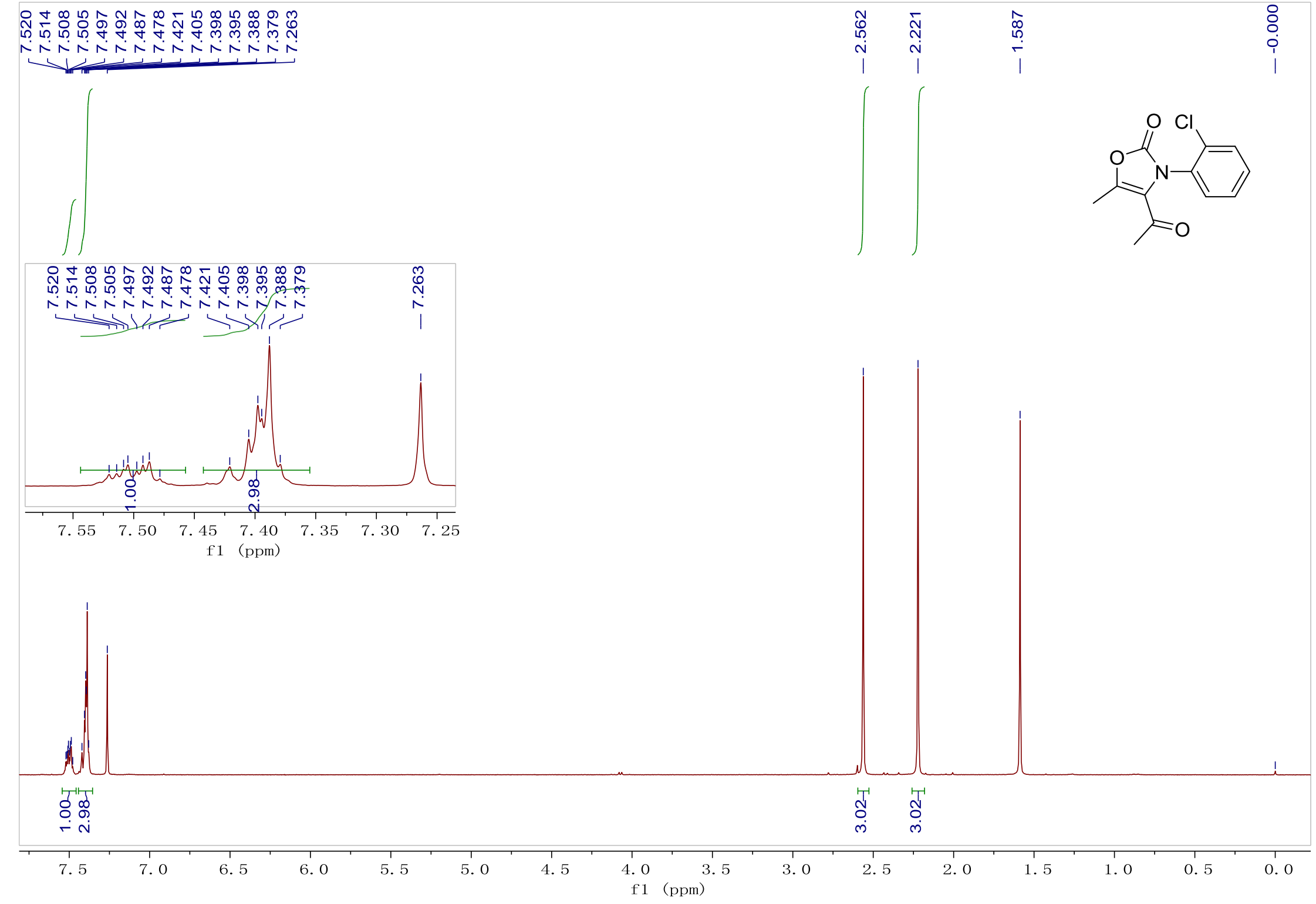


${ }^{13} \mathrm{C}\left\{{ }^{1} \mathrm{H}\right\}$ NMR spectrum of $\mathbf{4 g}\left(75 \mathrm{MHz}, \mathrm{CDCl}_{3}\right)$
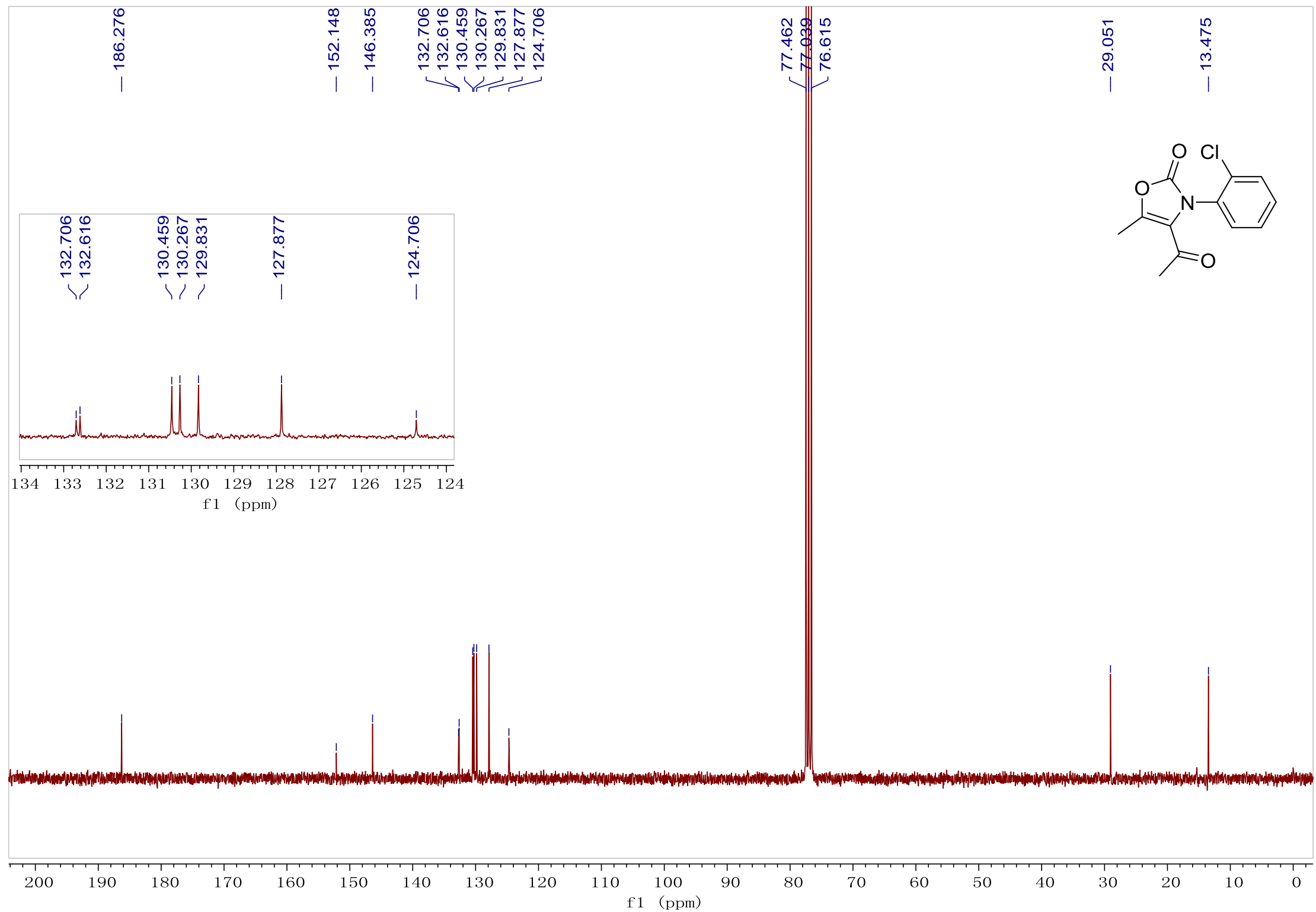
${ }^{1} \mathrm{H}$ NMR spectrum of $\mathbf{4 h}\left(300 \mathrm{MHz}, \mathrm{CDCl}_{3}\right)$

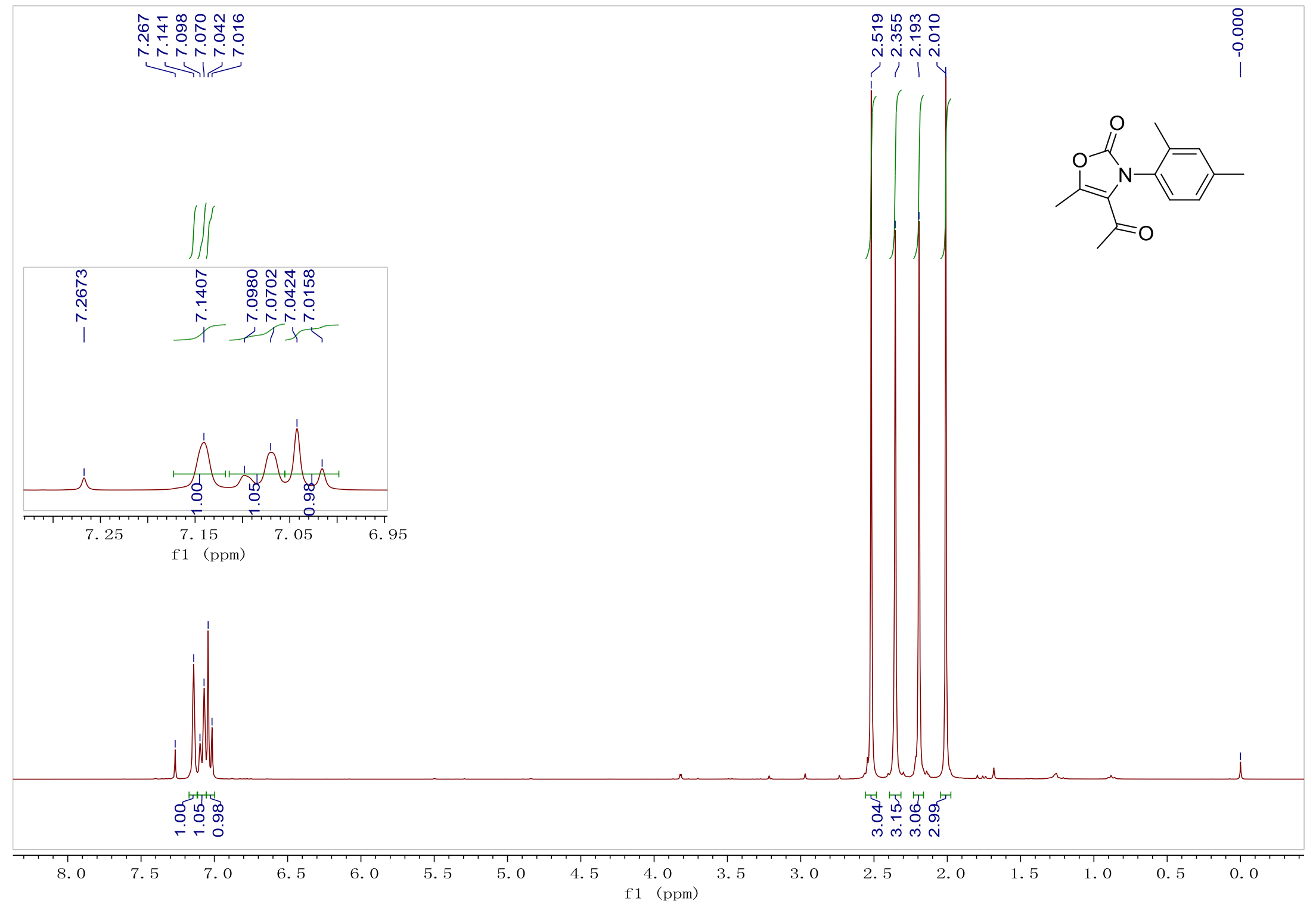


${ }^{13} \mathrm{C}\left\{{ }^{1} \mathrm{H}\right\}$ NMR spectrum of $\mathbf{4 h}\left(75 \mathrm{MHz}, \mathrm{CDCl}_{3}\right)$
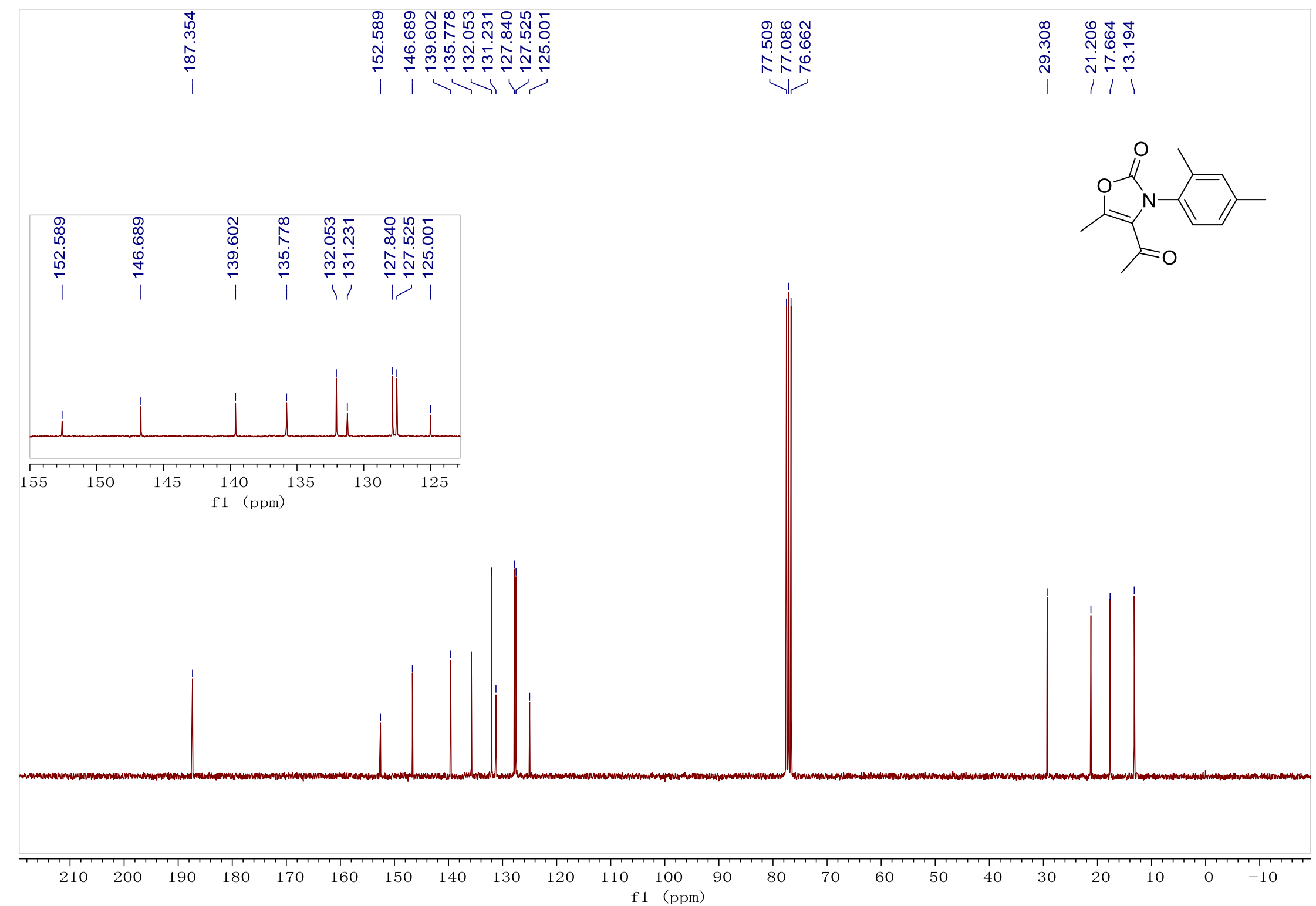
${ }^{1} \mathrm{H}$ NMR spectrum of $4 \mathbf{l}\left(300 \mathrm{MHz}, \mathrm{CDCl}_{3}\right.$ )

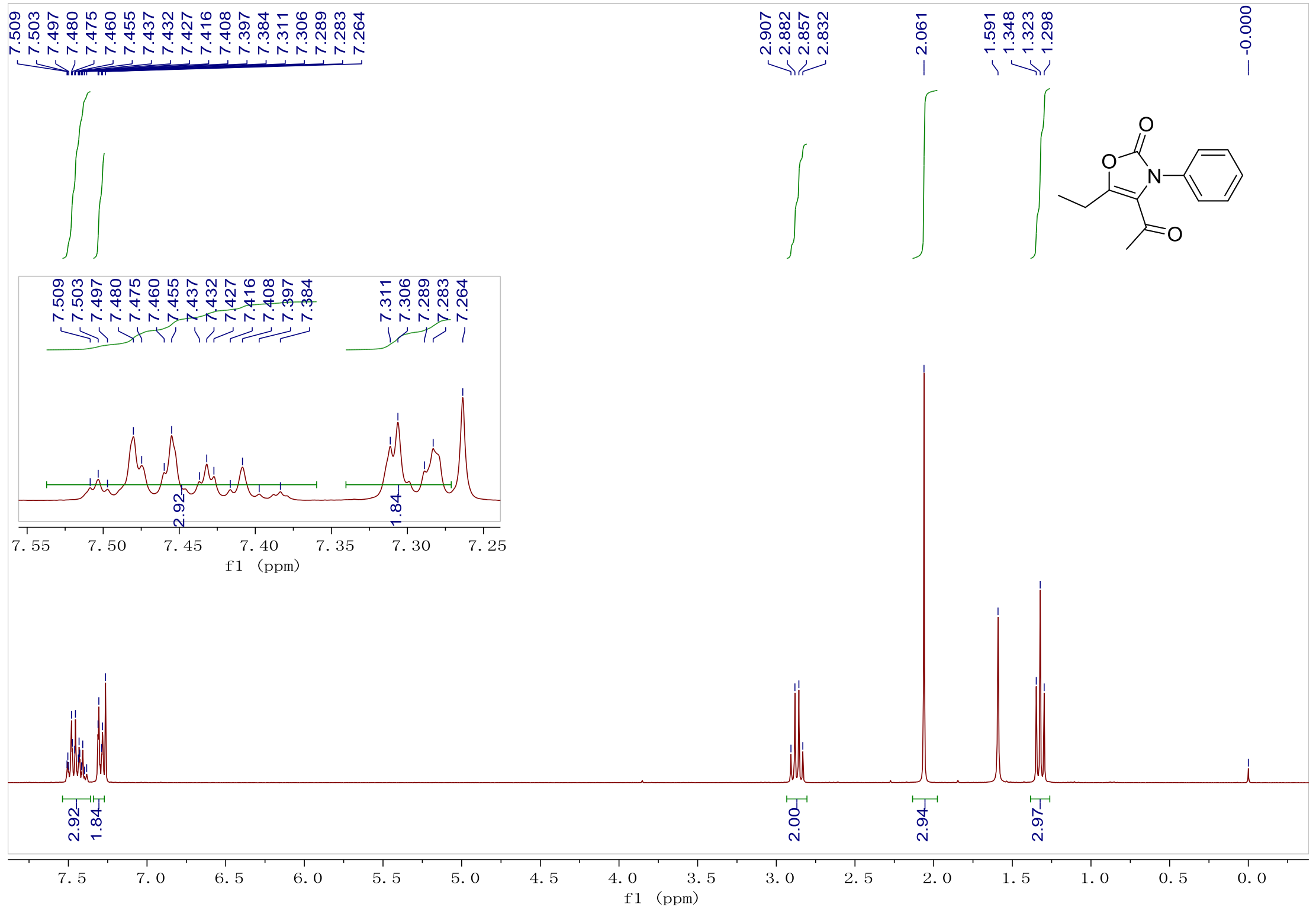

S59 
${ }^{13} \mathrm{C}\left\{{ }^{1} \mathrm{H}\right\}$ NMR spectrum of $4 \mathbf{l}\left(75 \mathrm{MHz}, \mathrm{CDCl}_{3}\right)$
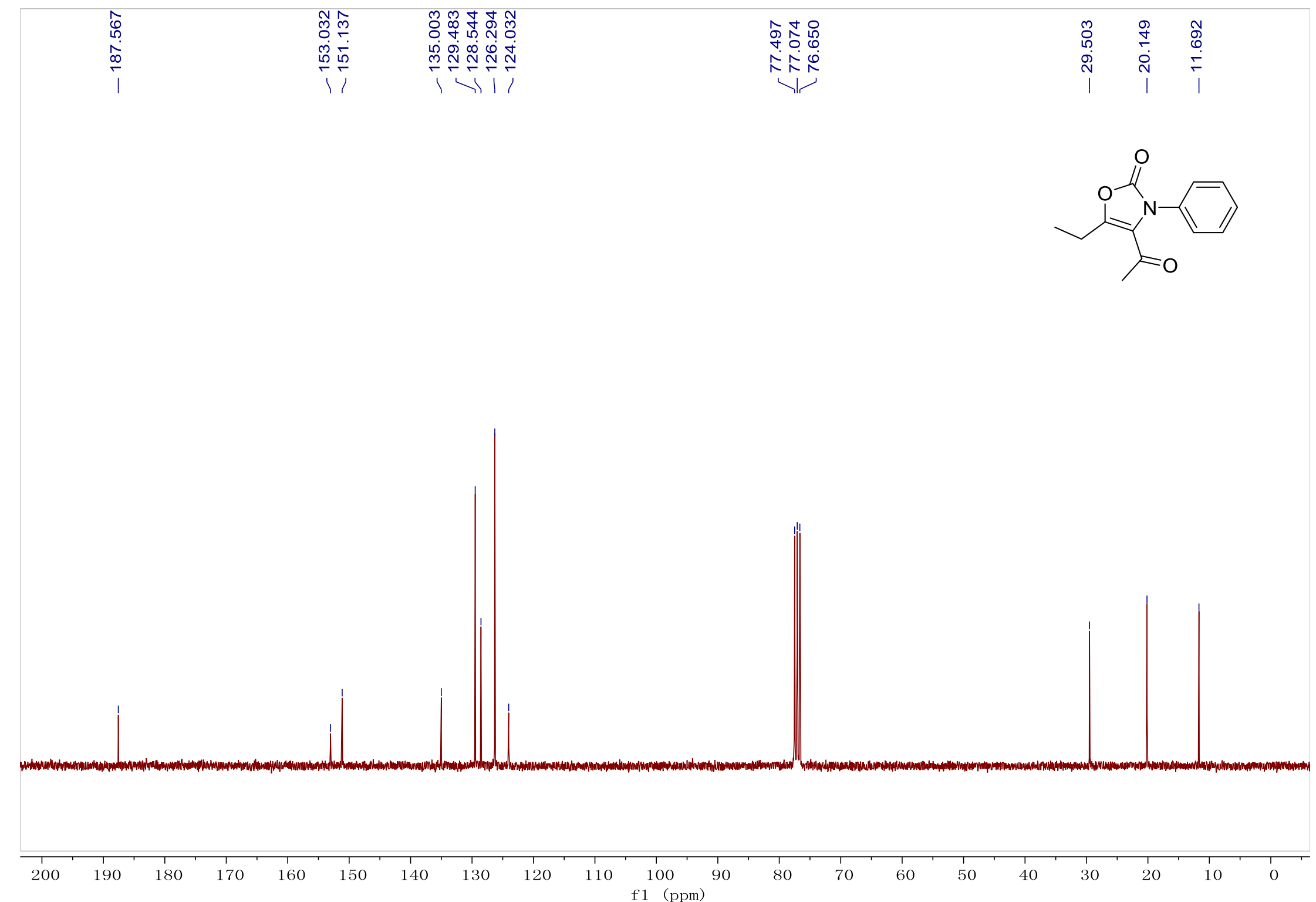
${ }^{1} \mathrm{H}$ NMR spectrum of $\mathbf{4 m}\left(300 \mathrm{MHz}, \mathrm{CDCl}_{3}\right)$

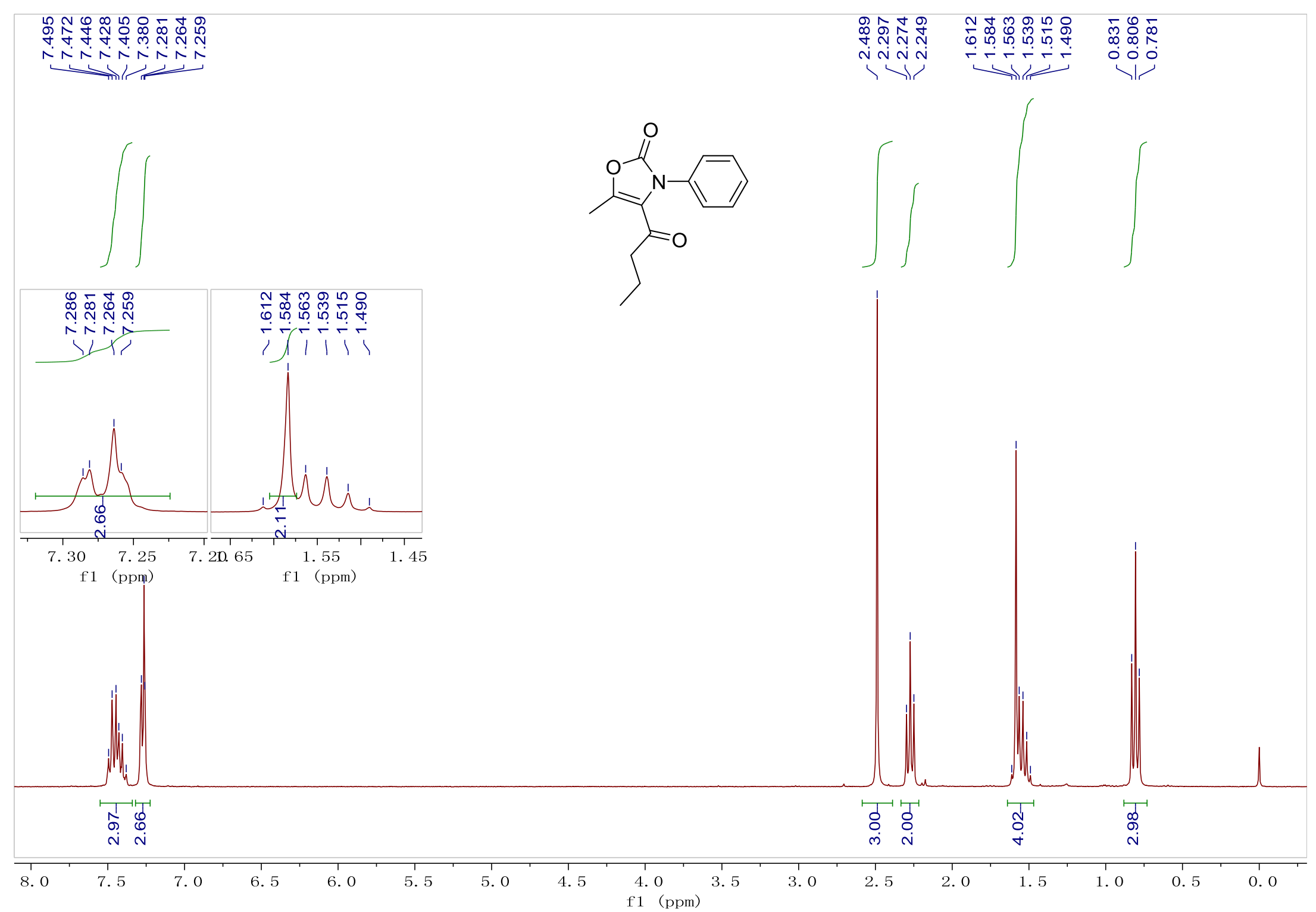


${ }^{13} \mathrm{C}\left\{{ }^{1} \mathrm{H}\right\}$ NMR spectrum of $\mathbf{4 m}\left(100 \mathrm{MHz}, \mathrm{CDCl}_{3}\right)$

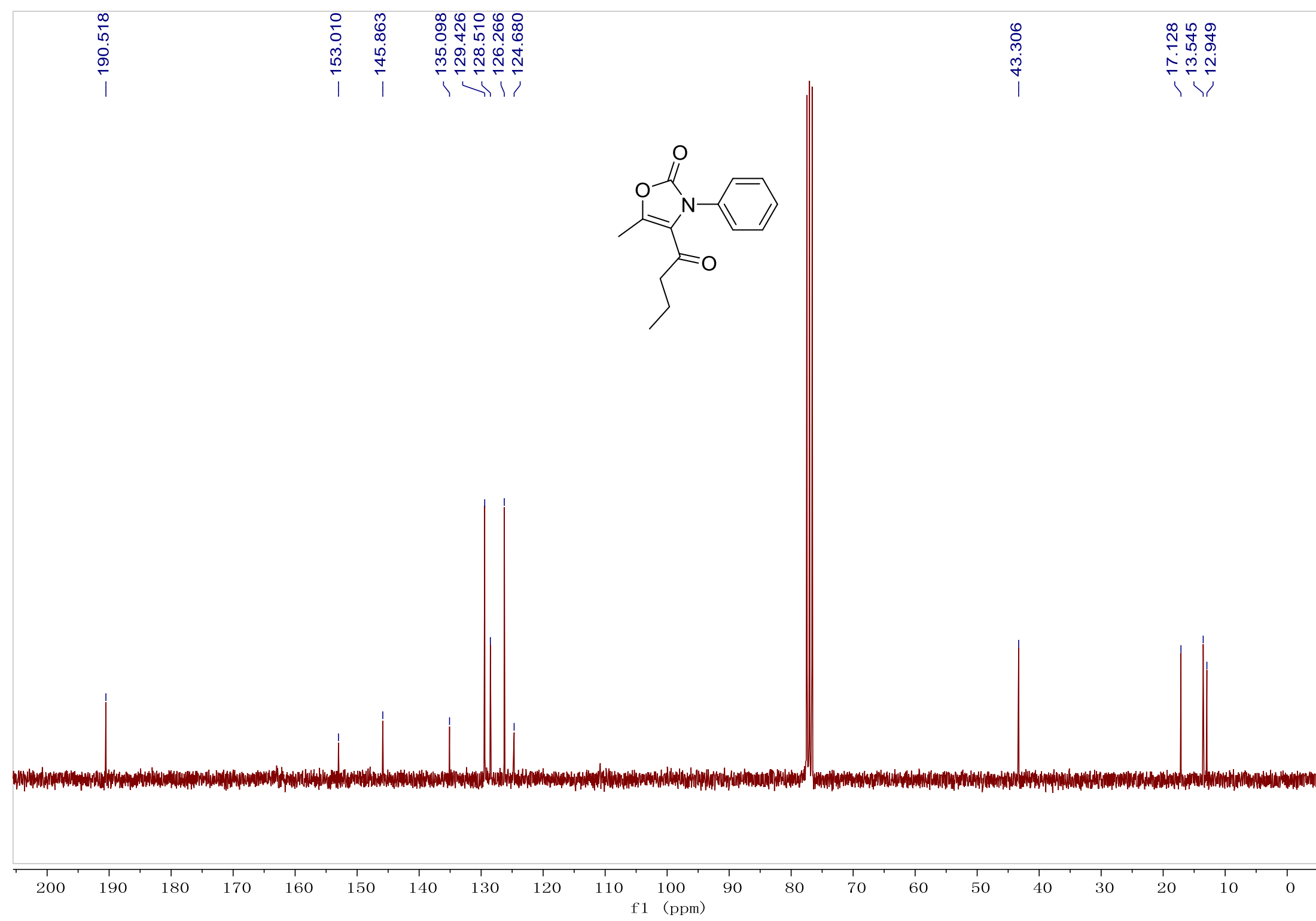

S62 
${ }^{1} \mathrm{H}$ NMR spectrum of $\mathbf{4 n}\left(300 \mathrm{MHz}, \mathrm{CDCl}_{3}\right)$

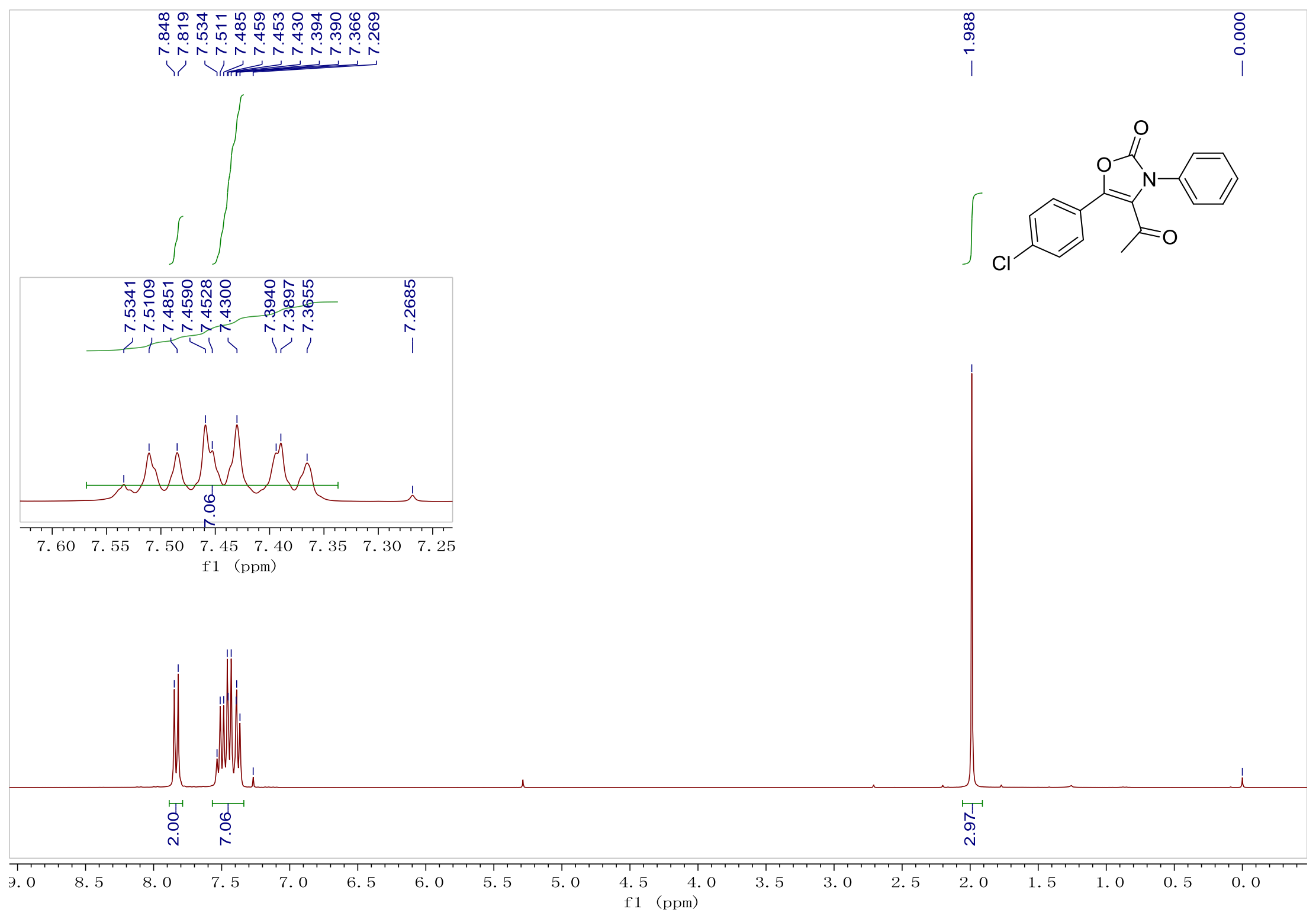


${ }^{13} \mathrm{C}\left\{{ }^{1} \mathrm{H}\right\}$ NMR spectrum of $4 \mathbf{n}\left(75 \mathrm{MHz}, \mathrm{CDCl}_{3}\right)$
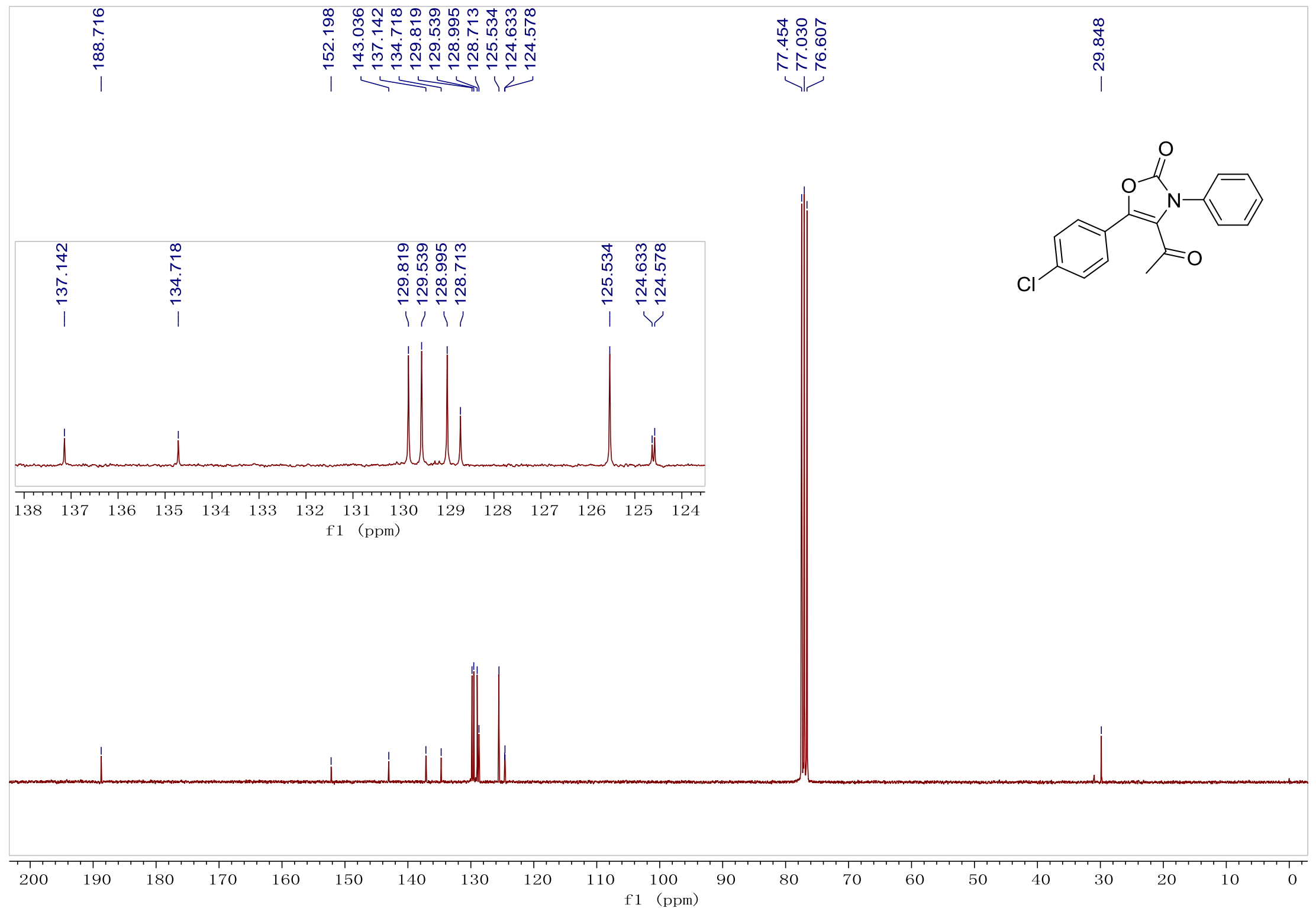
${ }^{1} \mathrm{H}$ NMR spectrum of $\mathbf{4 o}\left(300 \mathrm{MHz}, \mathrm{CDCl}_{3}\right)$

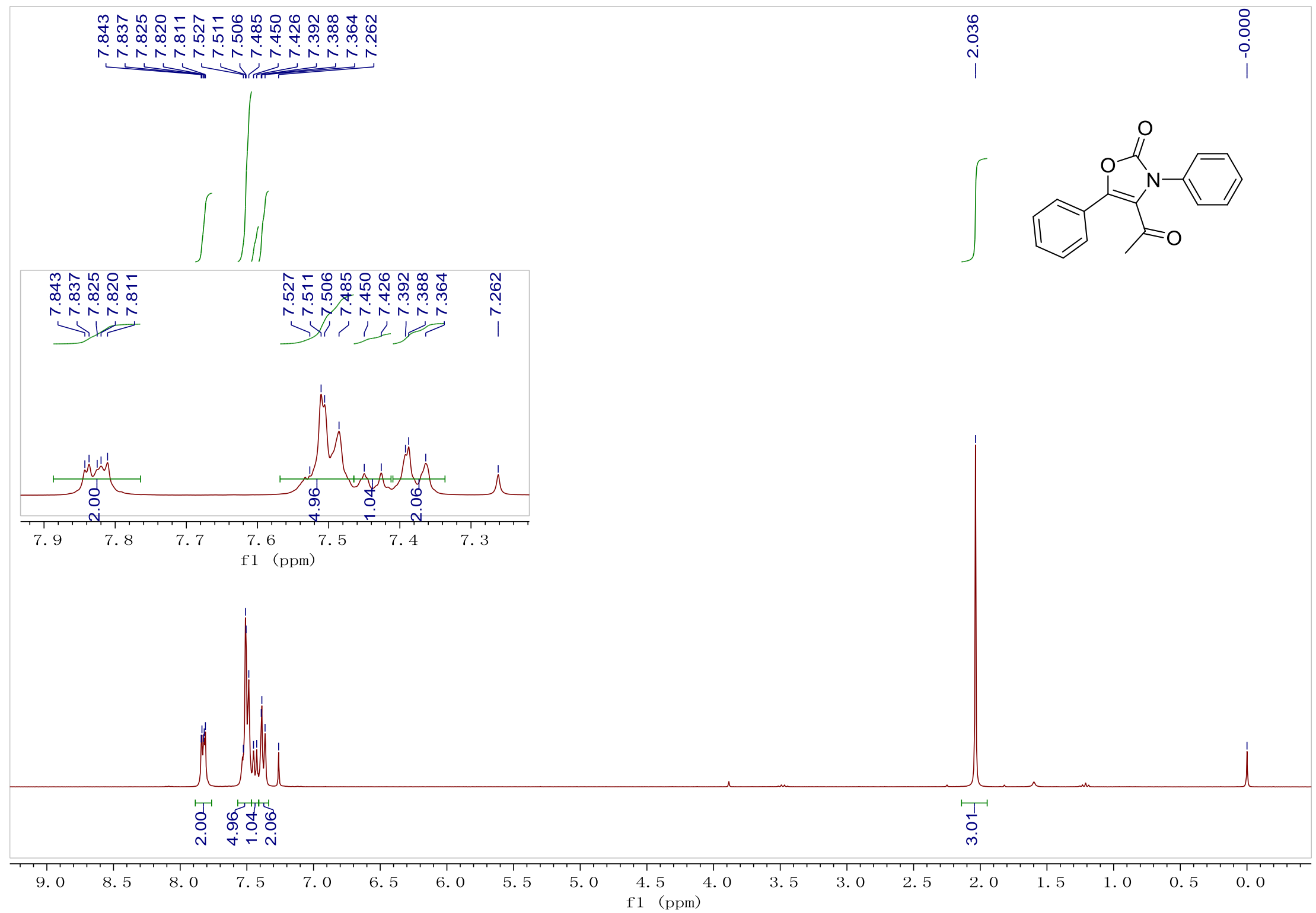

S65 
${ }^{13} \mathrm{C}\left\{{ }^{1} \mathrm{H}\right\}$ NMR spectrum of $40\left(100 \mathrm{MHz}, \mathrm{CDCl}_{3}\right)$
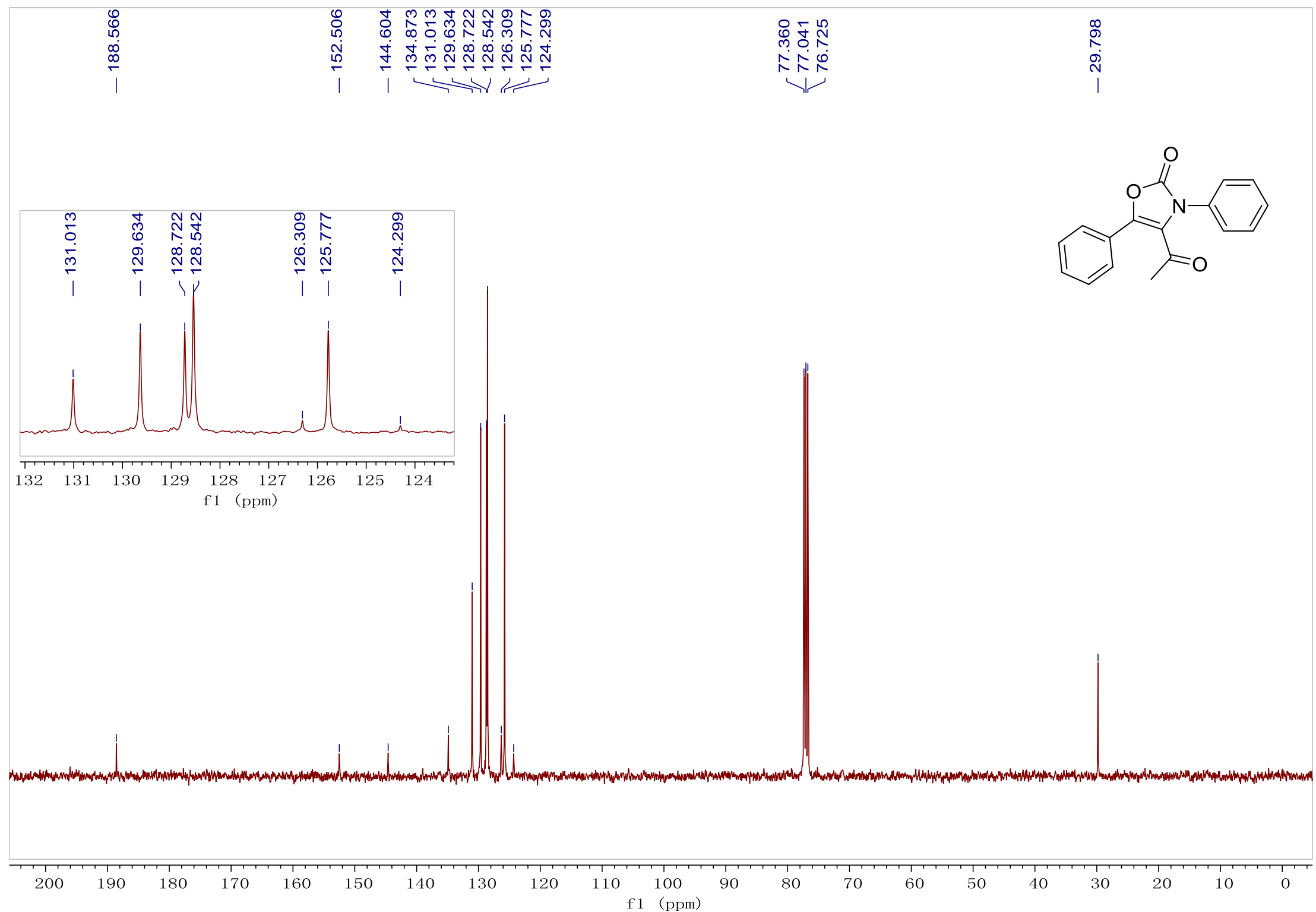
${ }^{1} \mathrm{H}$ NMR spectrum of $\mathbf{5 a}\left(300 \mathrm{MHz}, \mathrm{CDCl}_{3}\right)$

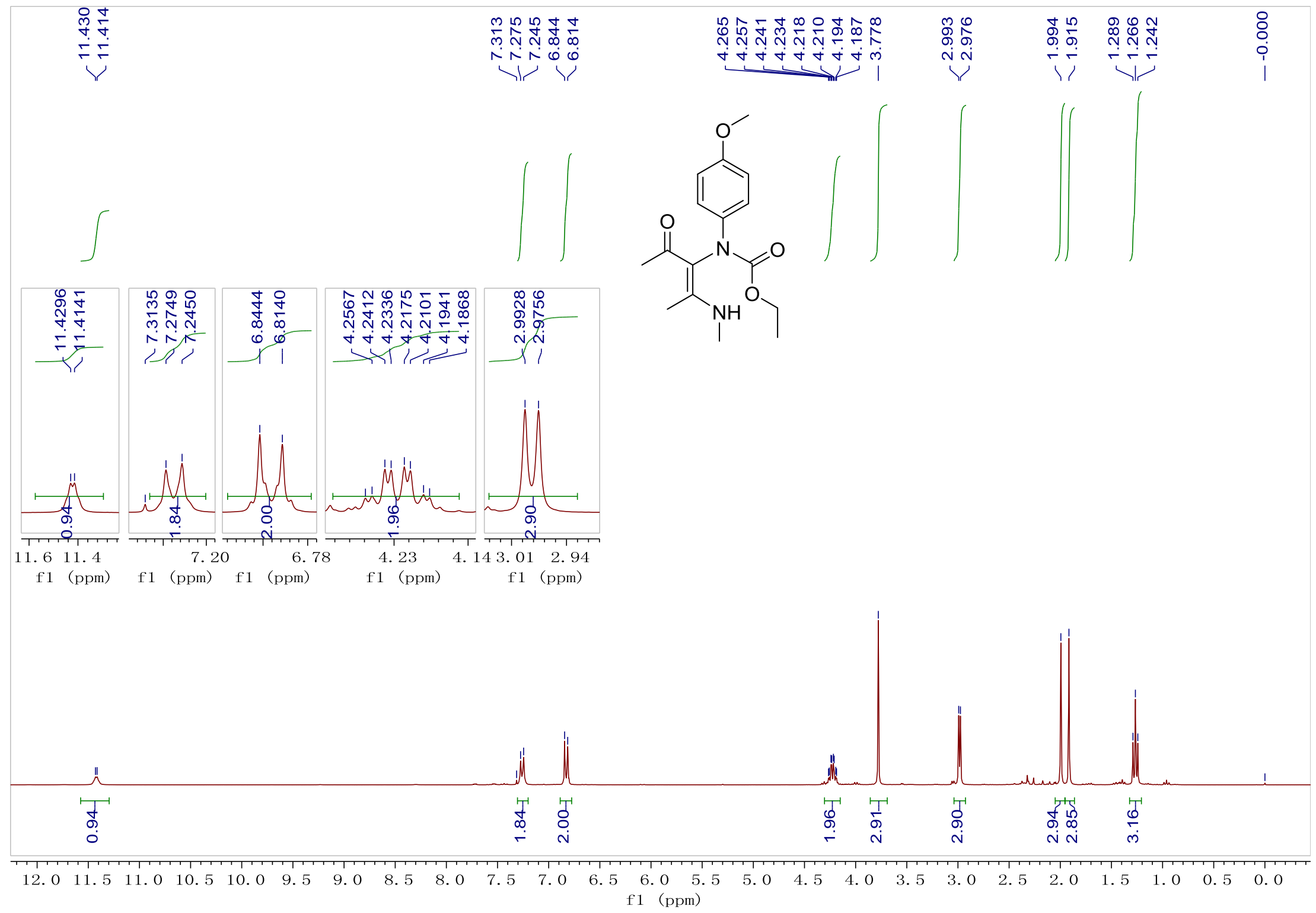


${ }^{13} \mathrm{C}\left\{{ }^{1} \mathrm{H}\right\}$ NMR spectrum of $\mathbf{5 a}\left(100 \mathrm{MHz}, \mathrm{CDCl}_{3}\right)$
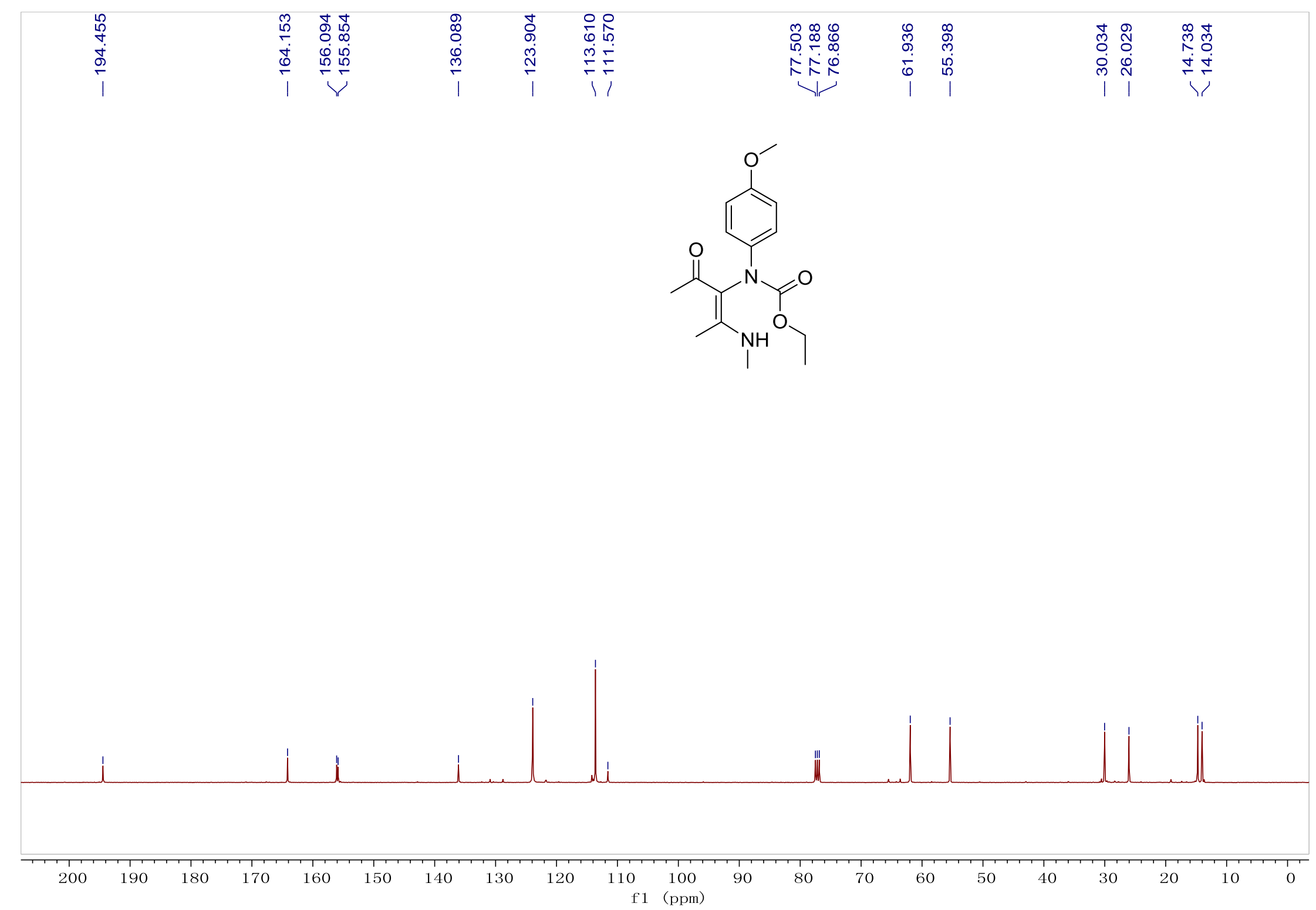
${ }^{1} \mathrm{H}$ NMR spectrum of $\mathbf{6 n}\left(300 \mathrm{MHz}, \mathrm{CDCl}_{3}\right)$

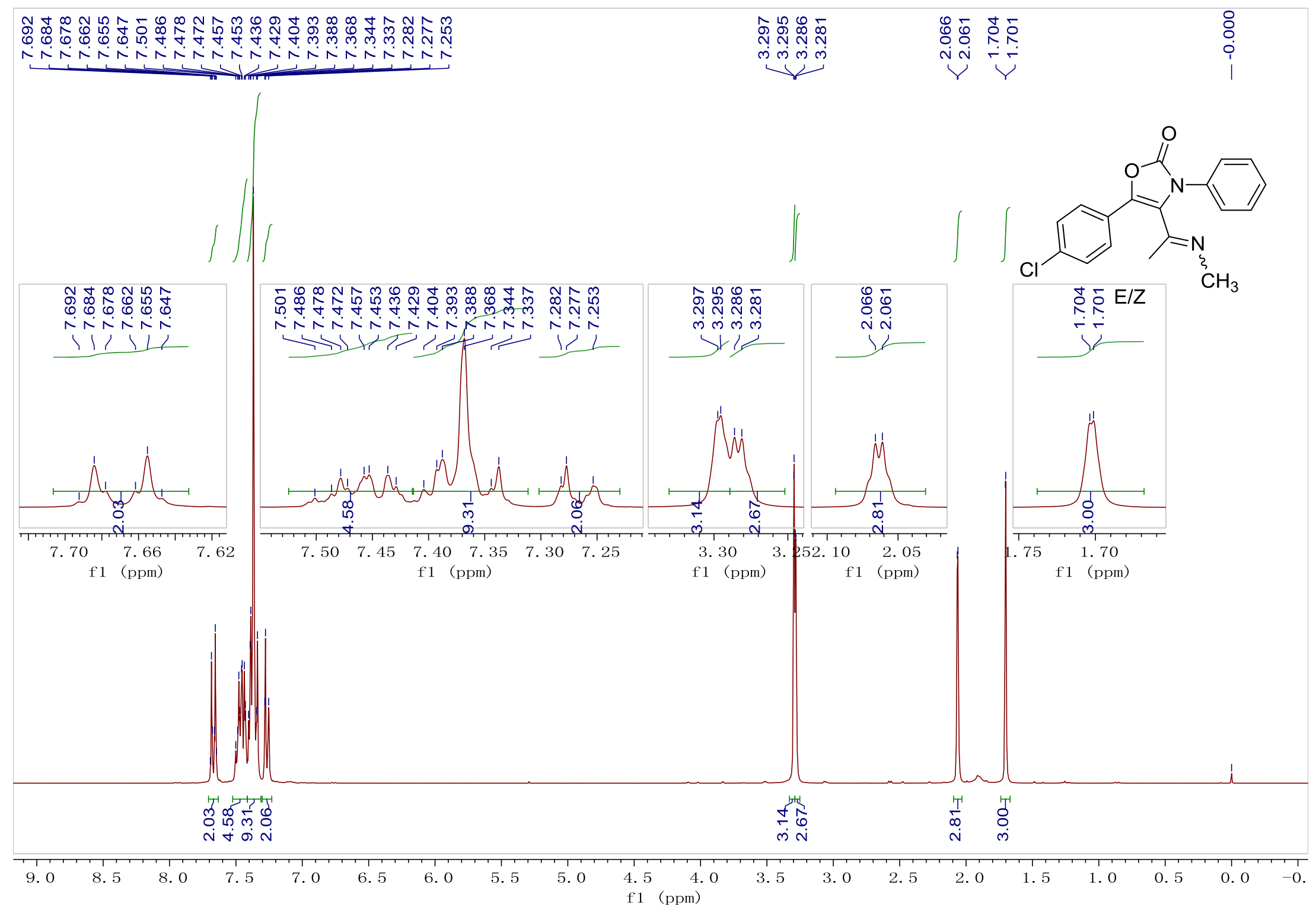


${ }^{13} \mathrm{C}\left\{{ }^{1} \mathrm{H}\right\}$ NMR spectrum of $6 \mathbf{n}\left(100 \mathrm{MHz}, \mathrm{CDCl}_{3}\right)$

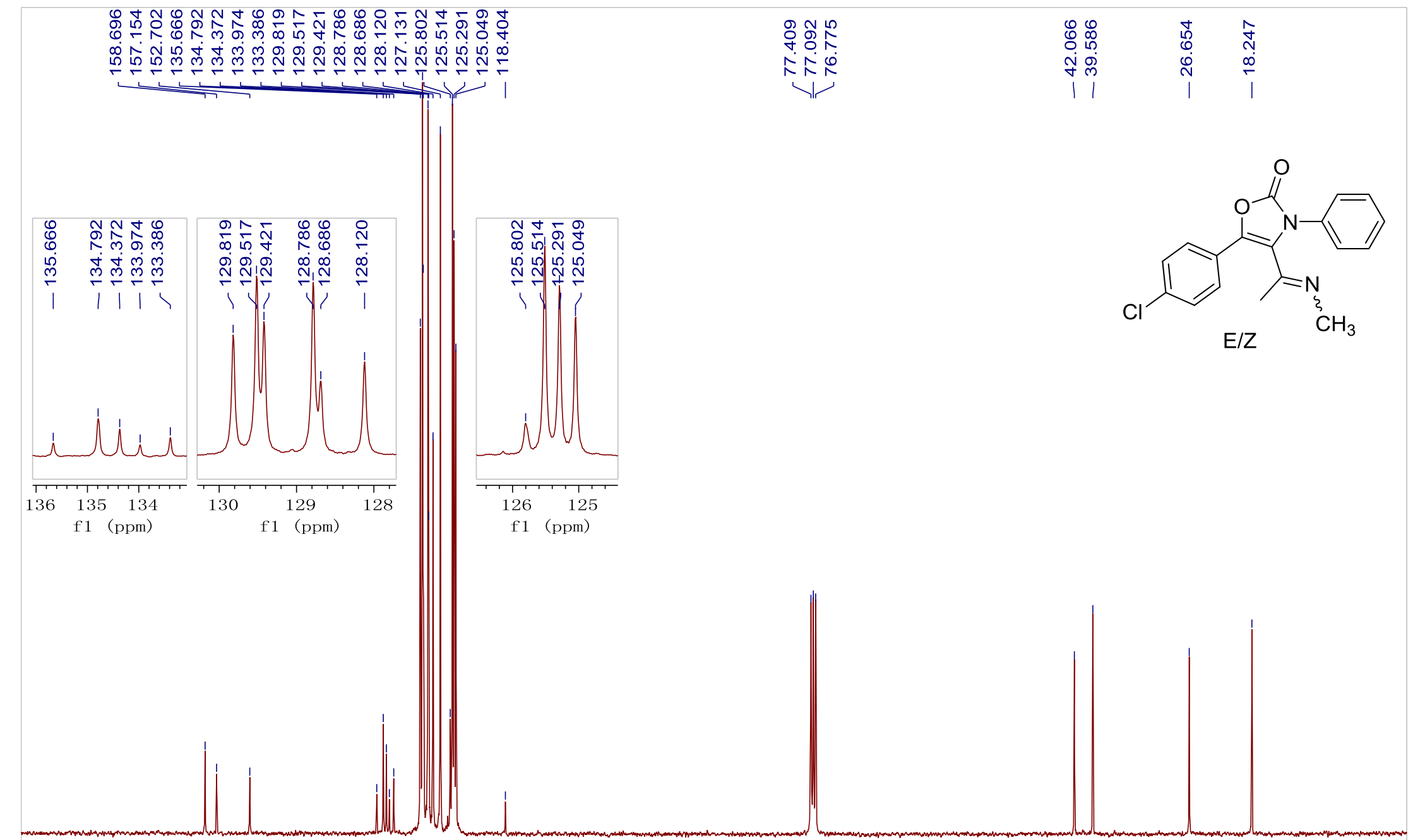

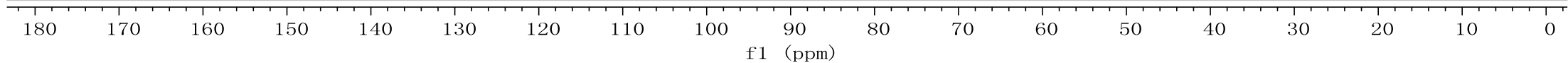


${ }^{1} \mathrm{H}$ NMR spectrum of $\mathbf{6 o}\left(300 \mathrm{MHz}, \mathrm{CDCl}_{3}\right)$

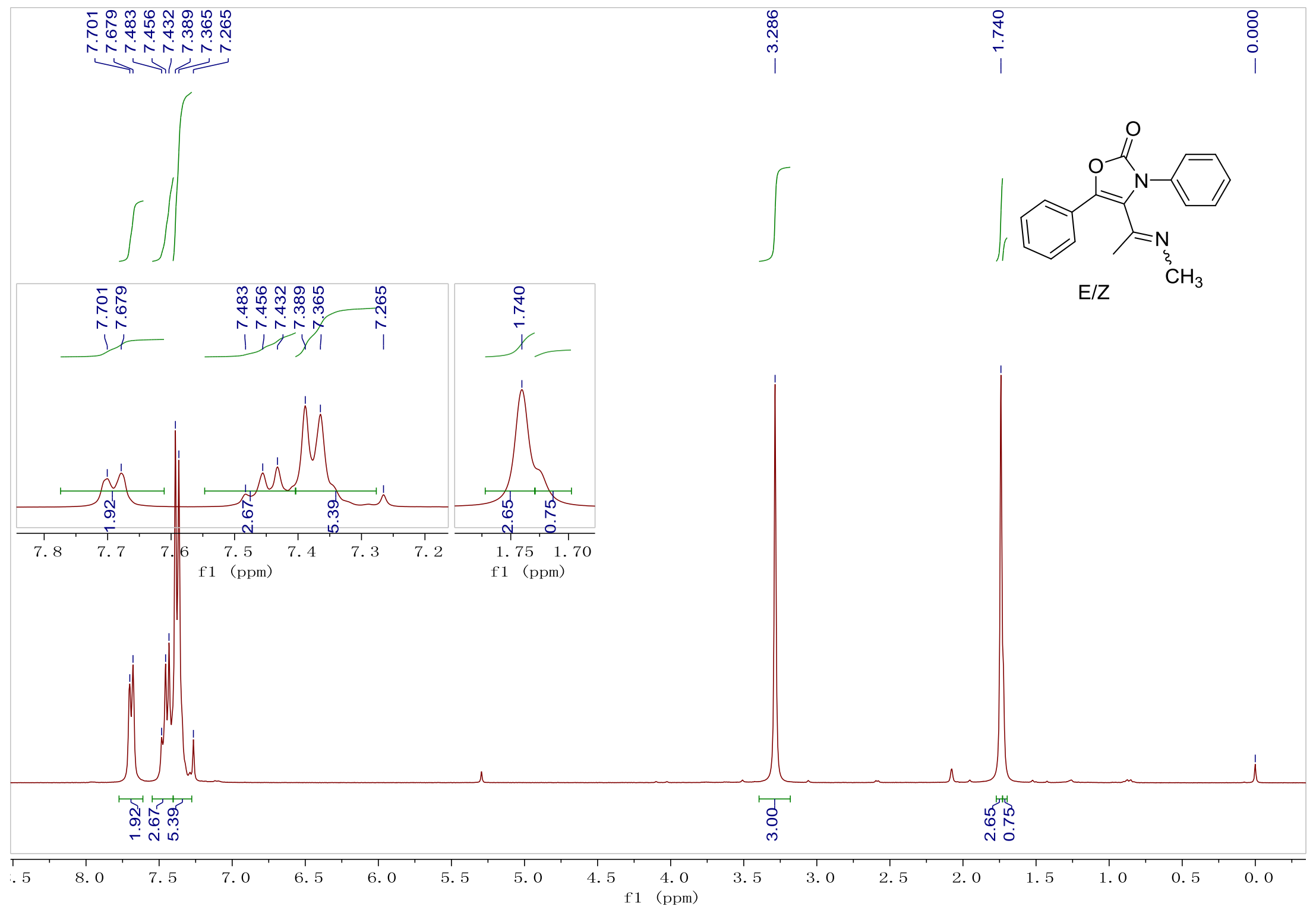


${ }^{13} \mathrm{C}\left\{{ }^{1} \mathrm{H}\right\}$ NMR spectrum of $\mathbf{6 o}\left(100 \mathrm{MHz}, \mathrm{CDCl}_{3}\right)$

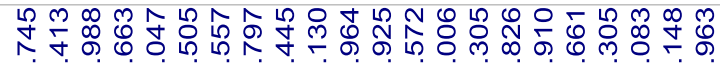

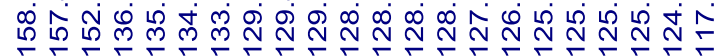

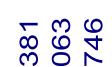

र下依

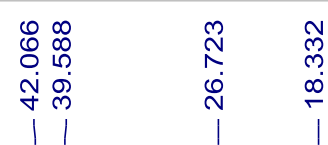

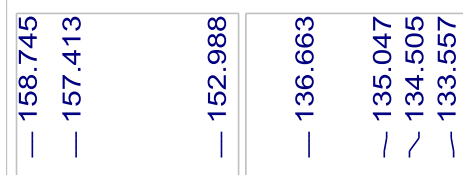
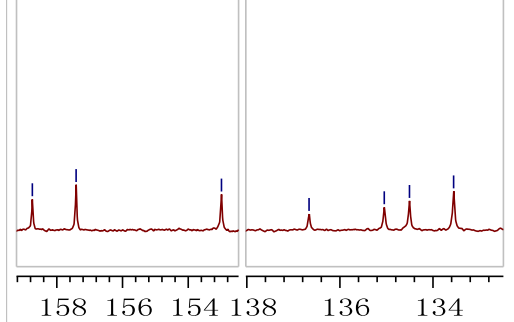

f1 (ppm)

f1 (ppm)

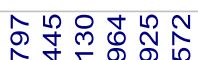

(1)

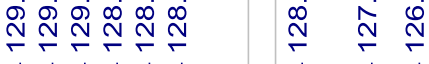

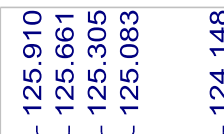

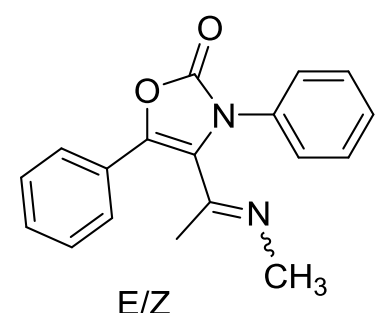

$\mid+E / Z$
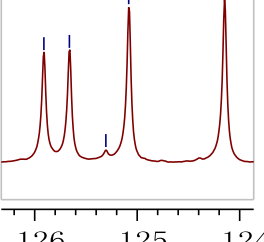

f1 (ppm)

f1 (ppm)

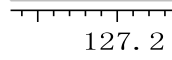

f1 (ppm)
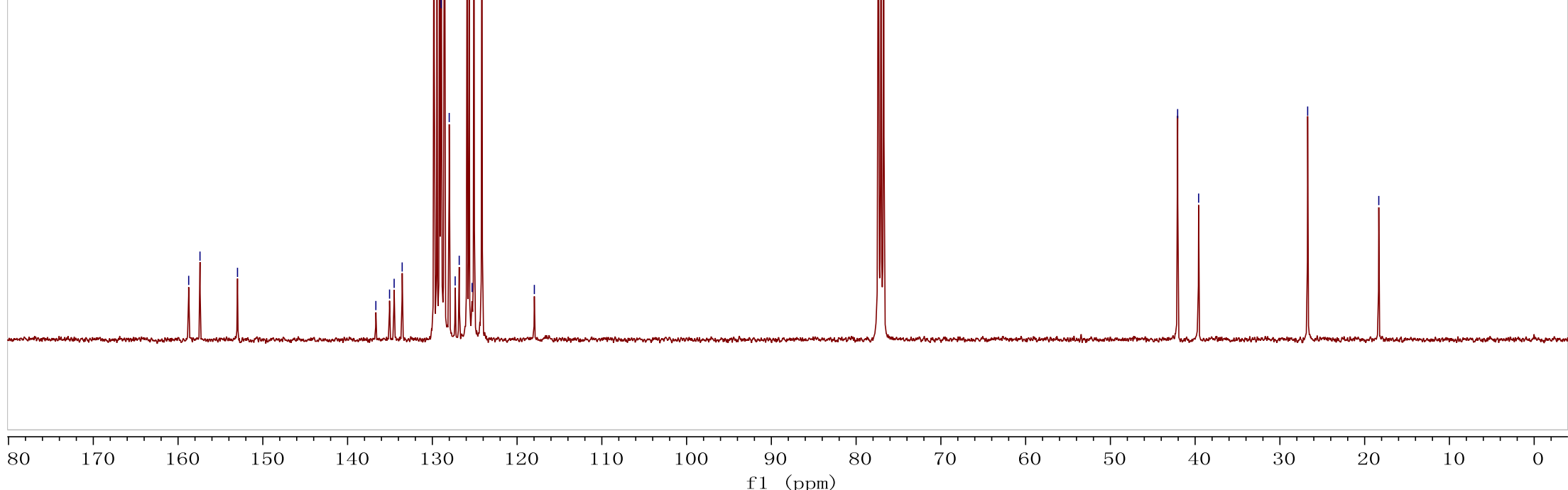